\title{
Synthesis of Sterically Hindered Ortho-substituted Tetraphenylethenes. Electronic Effects in the McMurry Olefination Reaction
}

\author{
Mee-Kyung Chung, Guizhong Qi, and Jeffrey M. Stryker* \\ Department of Chemistry, University of Alberta, Edmonton, Alberta Canada T6G $2 G 2$
}

\section{Supporting Information}

\section{General Experimental.}

Reagents and solvents. Manipulations of air sensitive compounds were performed under an argon atmosphere using standard Schlenk techniques or in nitrogen filled drybox equipped with an internal freezer maintained at $-35^{\circ} \mathrm{C}$. Tetrahydrofuran, 1, 2-dimethoxyethane, diethyl ether, hexane and pentane were dried and deoxygenated by distillation from sodium/benzophenone ketyl or sodium(potassium) alloy/benzophenone ketyl. Dichloromethane was dried by distillation from calcium hydride. $\mathrm{Zn}(\mathrm{Cu})$ couple was prepared by a literature procedure. ${ }^{1}$ The preparation of $\mathrm{TiCl}_{3} \bullet 1.5 \mathrm{dme}$ compound was based on the modification of a patent literature procedure. $^{2}$

Instruments. ${ }^{1} \mathrm{H}$ NMR and ${ }^{13} \mathrm{C}$ NMR spectra were recorded on Varian Inova 400 spectrometers. Unless stated otherwise, NMR spectra were obtained at room temperature $\left(23-27^{\circ} \mathrm{C}\right)$. Coupling constants $(J)$ are reported to $0.4 \mathrm{~Hz} /$ data point. Infrared spectra were recorded on a Nicolet Magna IR 750 spectrophotometer using compounds applied as a film to $\mathrm{KBr}$ or $\mathrm{KCl}$ salt plates (“casts") and are reported in wave numbers $\left(\mathrm{cm}^{-1}\right)$. High resolution mass spectra were obtained on a Kratos MS-50 spectrometer operating in electron impact mode (40-70 eV). Elemental (combustion) analyses were performed using a Carlo Erba (EA1108) elemental analyzer by the University of Alberta Microanalysis Laboratory. X-ray diffraction data were collected on a Bruker SMART $1000 \mathrm{CCD}$ detector/PLATFORM diffractometer at $-80{ }^{\circ} \mathrm{C}$ with $\mathrm{Mo} \mathrm{K}$ radiation 
(vide infra). Column chromatography was performed with E. Merck silica gel (230-400 mesh). Visualization of TLC plates was accomplished using Hanessian's cerium molybdate stain. ${ }^{3}$

Preparation of starting materials. Synthesis of substituted diarylketones. Bis(5-tert-butyl2-methoxyphenyl)methanone (1a), ${ }^{4}$ 2,2'-dimethoxybenzophenone (1b), ${ }^{4}$ di-ortho-tolylketone (1d), ${ }^{5}$ and bis(4-methoxy-2-methylphenyl)methanone $(\mathbf{1 e})^{6}$ were synthesized by following literature procedures. (2-Methoxyphenyl)(2-methylphenyl)methanone (1c) was prepared from $N$-methoxy- $N$, 2-dimethyl benzamide ${ }^{7}$ (the Weinreb amide ${ }^{8}$ )and $o$-methoxyphenyllithium, according to the published procedure. ${ }^{9}$ 2,4'-Dimethoxybenzophenone (1f) was also prepared from the Weinreb amide $N$, 2-dimethoxy- $N$-methyl benzamide ${ }^{7}$ and $p$-methoxyphenyllithium. ${ }^{9}$ Commercially available 2,4-dimethoxybenzophenone (1g) was synthesized by the Friedel-Crafts acylation reaction of 1, 3-dimethoxybenzene and benzoyl chloride. ${ }^{10}$ 2-Methoxybenzophenone (1h) was purchased from Aldrich and used as received. (2-Methoxyphenyl)[4-(trifluoromethyl)phenyl]methanone (1i) was prepared from $N$, 2-dimethoxy- $N$-methyl benzamide ${ }^{7}$ and $p$ trifluoromethylphenyllithium, which was prepared as reported. ${ }^{11}$

\section{McMurry coupling reaction of substituted diarylketones.}

\section{(i) Optimized general procedure for the McMurry coupling reaction (as represented by}

Table 1, entry 4). This protocol was also used for the McMurry reaction of the diarylmethanone substrates reported in Table $\mathbf{2}$ and $\mathbf{3}$, all of which were conducted under identical conditions. All operations were performed under an atmosphere of argon. A slurry of $\mathrm{TiCl}_{3}(0.62 \mathrm{~g}, 4.0 \mathrm{mmol}$, 1.0 equiv) and $\mathrm{Zn}(\mathrm{Cu})(0.13 \mathrm{~g}, 2.0 \mathrm{mmol}, 0.5$ equiv) in anhydrous 1,2-dimethoxyethane (DME, $12 \mathrm{~mL}$ ) was heated to reflux for $14 \mathrm{~h}$. The reaction mixture was then cooled to ambient temperature. A separately prepared solution of the diarylketone ( $0.68 \mathrm{mmol}, 0.17$ equiv) in DME (6 mL) was transferred to the cooled slurry of low-valent titanium and the resulting 
reaction mixture stirred at ambient temperature. The progress of the reaction was monitored by analytical TLC. After completion of the reaction, the resultant suspension was filtered through a Celite pad, the solids were washed with dichloromethane or diethyl ether, and the combined organic material was concentrated in vacuo. Purification by flash column chromatography on silica gel using a gradient solvent system composed of $\mathrm{CH}_{2} \mathrm{Cl}_{2} /$ hexanes provided the isolated alkene derivative.

For the table entries conducted using $\mathrm{LiAlH}_{4}$ as the reductant, the active titanium reagent was generated at ambient temperature (Table 1, entries 1-3 ). For the entries in which the reagent preparation step is omitted, $\mathrm{TiCl}_{3}(0.62 \mathrm{~g}, 4.0 \mathrm{mmol}, 1.0$ equiv) and $\mathrm{Zn}(\mathrm{Cu})(0.13 \mathrm{~g}, 2.0 \mathrm{mmol}$, 0.5 equiv) were suspended in DME ( $12 \mathrm{~mL})$, and the separately prepared solution of the diarylketone ( $0.68 \mathrm{mmol}, 0.17$ equiv) in DME $(6 \mathrm{~mL})$ was added at ambient temperature; the reaction itself proceeded either at ambient temperature or at reflux (Table $\mathbf{1}$, entry $\mathbf{5}$ ).

(ii) Tetrakis(5-tert-butyl-2-methoxyphenyl)ethene (2a): large scale experiment (Table 1, entry 14). An oven-dried, $1 \mathrm{~L}$ Schlenk flask was charged with titanium trichloride (24.69 g, $160.0 \mathrm{mmol})$ and $\mathrm{Zn}(\mathrm{Cu})(5.16 \mathrm{~g}, 79.0 \mathrm{mmol})$ in the glove box. After removal from the box, anhydrous DME $(350 \mathrm{~mL})$ was added under an argon flow and the flask affixed with a condenser connected to a gas bubbler with a counter-current of argon. The resulting reaction mixture (slurry) was heated to reflux for $14 \mathrm{~h}$ using an oil bath maintained at $100-110{ }^{\circ} \mathrm{C}$ and then cooled to ambient temperature. A separately prepared solution of bis(5-tert-butyl-2methoxyphenyl)methanone (9.72 g, $27.4 \mathrm{mmol})$ in anhydrous DME $(130 \mathrm{~mL})$ was transferred to the cooled slurry of low valent titanium and the resultant reaction mixture stirred at ambient temperature. The progress of the reaction was monitored by analytical TLC. After completion of the reaction, the volume of solution was reduced in vacuo by one-thirds and the reaction cautiously quenched with $4 \mathrm{~mL}$ of $1.0 \mathrm{M}$ aqueous $\mathrm{HCl}$ followed by $200 \mathrm{~mL}$ of water. The resulting mixture was extracted three times with $100 \mathrm{~mL}$ of diethyl ether. The combined organic layers were dried over $\mathrm{MgSO}_{4}$ and the volatiles removed on the rotary evaporator. The crude 
product was crystallized from $10 \% \mathrm{CH}_{2} \mathrm{Cl}_{2}$ in pentane and isolated by filtration and drying under vacuum, affording $6.20 \mathrm{~g}$ of tetrakis(5-tert-butyl-2-methoxyphenyl)ethene (2a) as an air-stable white solid. The residue from the mother liquor was purified by column chromatography on silica gel using a gradient solvent system of $\mathrm{CH}_{2} \mathrm{Cl}_{2} /$ hexanes, providing an additional $1.56 \mathrm{~g}$ of purified product (combined yield 84\%, based on bis(5-tert-butyl-2-methoxypheny)methanone).

For the larger scale synthesis of tetrakis(2-dimethoxyphenyl)ethene (2b) from 2,2'dimethoxybenzophenone (1b) following this procedure $(4.12 \mathrm{~g}, 17.0 \mathrm{mmol}$ of ketone), a yield of $73 \%$ of purified $\mathbf{2} \mathbf{b}$ was obtained.

(iii) Tetrakis(5-tert-butyl-2-methoxyphenyl)ethene (2a): $\mathrm{TiCl}_{3} \cdot 1.5 \mathrm{dme}^{2}$ as the titanium source (Table 1, entry 12). The reaction of the Ti(III) reagent and $\mathrm{Zn}(\mathrm{Cu})$ in $\mathrm{DME}$ at reflux (the McMurry reagent preparation step) was unnecessary for optimum results and is omitted from this procedure. ${ }^{12}$ In addition, the molar excess of titanium was reduced by about one-half compared to procedure (ii), increasing the efficiency of this fully optimized protocol. All operations were performed under an atmosphere of argon. An oven-dried, 1L Schlenk flask was charged with $\mathrm{TiCl}_{3} \bullet 1.5 \mathrm{dme}(13.27 \mathrm{~g}, 45.9 \mathrm{mmol})$ and $\mathrm{Zn}(\mathrm{Cu})(1.50 \mathrm{~g}, 23.0 \mathrm{mmol})$ in the glove box. After removal from the box, anhydrous DME $(350 \mathrm{~mL})$ was added under an argon flow. A separately prepared solution of bis(5-tert-butyl-2-methoxyphenyl)methanone (6.0 g, $16.9 \mathrm{mmol})$ in anhydrous DME $(80 \mathrm{~mL})$ was transferred to the slurry of $\mathrm{TiCl}_{3} \bullet 1.5 \mathrm{dme} / \mathrm{Zn}(\mathrm{Cu})$ and the resultant reaction mixture stirred at ambient temperature for 2 days, with the progress of the reaction monitored by analytical TLC. After completion of the reaction, in the same way as described in the procedure (ii), the reaction was quenched with $1.0 \mathrm{M}$ aqueous $\mathrm{HCl}$ followed by the addition of water. After the extraction of the resulting mixture with diethyl ether $(3 \times 100$ $\mathrm{ml}$ ), the combined organic layers were dried over $\mathrm{MgSO}_{4}$ and the volatiles removed on the rotary evaporator. The crude product was crystallized from $10 \% \mathrm{CH}_{2} \mathrm{Cl}_{2}$ in pentane, affording $3.42 \mathrm{~g}$ of tetrakis(5-tert-butyl-2-methoxyphenyl)ethene (2a) as an air-stable white solid. The residue from the mother liquor was purified by column chromatography on silica gel using a 
gradient solvent system of $\mathrm{CH}_{2} \mathrm{Cl}_{2} /$ hexanes, providing an additional $1.13 \mathrm{~g}$ of purified product (combined yield 79\%, based on bis(5-tert-butyl-2-methoxypheny)methanone).

(iv) Control reaction. Conducted by adding tetrakis(5-tert-butyl-2-methoxyphenyl)ethene (2a) to the McMurry reaction of 2,2'-dimethoxybenzophenone (1b). All operation were performed under an atmosphere of argon. A slurry of $\mathrm{TiCl}_{3}(0.62 \mathrm{~g}, 4.0 \mathrm{mmol}, 1.0$ equiv) and $\mathrm{Zn}(\mathrm{Cu})(0.13 \mathrm{~g}, 2.0 \mathrm{mmol}, 0.5$ equiv) in DME (12.0 mL) was heated to reflux for $14 \mathrm{~h}$. The reaction mixture was then cooled to ambient temperature. A separately prepared solution of 2,2'-dimethoxybenzophenone $(0.082 \mathrm{~g}, 0.34 \mathrm{mmol}, 0.085$ equiv $)$ and $\mathbf{2 a}(0.12 \mathrm{~g}, 0.17 \mathrm{mmol}$, 0.043 equiv) in DME (6.0 mL) was transferred to the cooled slurry of low-valent titanium and the resulting reaction mixture stirred at ambient temperature. The progress of the reaction was monitored by analytical TLC. After $24 \mathrm{~h}$, the reaction quenched by the careful addition of $5 \mathrm{~mL}$ of water. The resulting reaction mixture was extracted three times with $10 \mathrm{~mL}$ of diethyl ether. The combined organic layers were dried over $\mathrm{MgSO}_{4}$ and the volatiles removed on the rotary evaporator. ${ }^{1} \mathrm{H}$ NMR spectroscopy of the crude products indicated that the reaction generated no tetrakis(5-tert-butyl-2-methoxyphenyl)ethane (3a), the product expected from reduction of

the control. Signals were observed for the control substrate $\mathbf{2 a}$, as well as mixture of tetrakis(2methoxyphenyl)ethene (2b) and tetrakis(2-methoxyphenyl)ethane (3b), and traces of additional materials, i.e., only the McMurry reaction products formed from the coupling of 2,2'dimethoxybenzophenone alone.

Spectroscopic and analytical data for the compounds reported in Table 2. For known compounds referenced to the literature, only partial and/or updated data are included.

Tetrakis(5-tert-butyl-2-methoxyphenyl)ethene $(2 \mathrm{a})^{4}$ and 1,1,2,2-Tetrakis(5-tert-butyl-2methoxyphenyl)ethane (3a). 


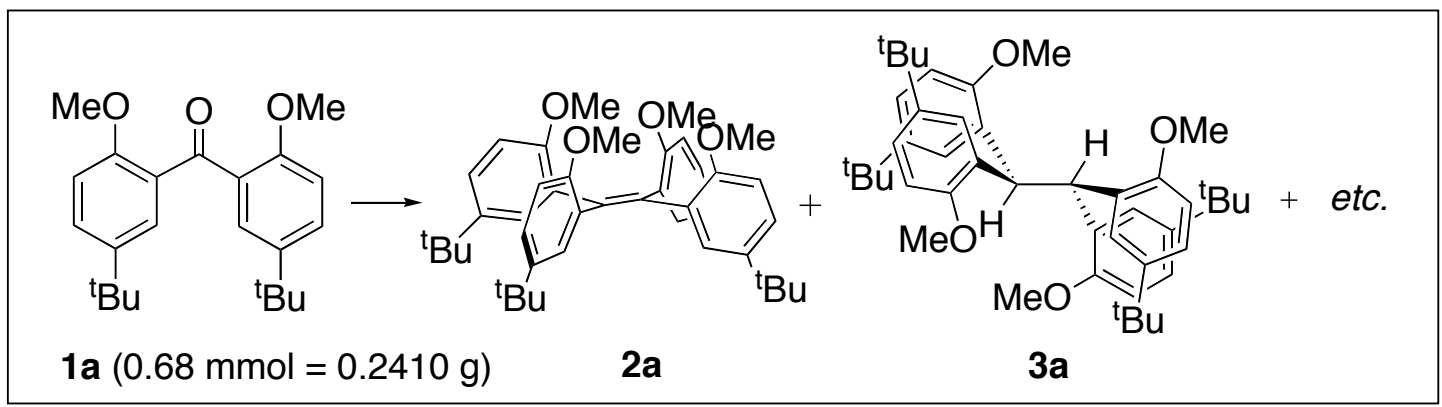

Chromatography conditions: gradient consisting of (i) 100\% hexanes (100 ml), (ii) 30\% $\mathrm{CH}_{2} \mathrm{Cl}_{2} /$ hexanes $(300 \mathrm{ml})$, and (iii) $100 \% \mathrm{CH}_{2} \mathrm{Cl}_{2}(400 \mathrm{ml})$.

Tetrakis(5-tert-butyl-2-methoxyphenyl)ethene (2a). ${ }^{4}$ TLC $R_{f} 0.23\left(50 \% \mathrm{CH}_{2} \mathrm{Cl}_{2} /\right.$ hexanes). ${ }^{1} \mathrm{H}$ NMR (400 MHz, $\left.\mathrm{CDCl}_{3}\right) \delta 1.07(\mathrm{~s}, 36 \mathrm{H}), 3.48(\mathrm{~s}, 12 \mathrm{H}), 6.56(\mathrm{~d}, J=8.8 \mathrm{~Hz}, 4 \mathrm{H}), 6.93(\mathrm{dd}, J=$ 8.4, $2.4 \mathrm{~Hz}, 4 \mathrm{H}), 7.09(\mathrm{~d}, J=2.4 \mathrm{~Hz}, 4 \mathrm{H})$.

1,1,2,2-Tetrakis(5-tert-butyl-2-methoxyphenyl)ethane (3a). TLC $R_{f} 0.54$ (50\% $\mathrm{CH}_{2} \mathrm{Cl}_{2} /$ hexanes). ${ }^{1} \mathrm{H} \mathrm{NMR}\left(400 \mathrm{MHz}, \mathrm{CDCl}_{3}\right) \delta 1.19$ (s, 36H), 3.66 (s, 12H), 5.83 (s, 2H), 6.54 (d, J = 8.8 Hz, 4H), $6.93(\mathrm{dd}, J=8.4,2.8 \mathrm{~Hz}, 4 \mathrm{H}), 7.45(\mathrm{~s}, 4 \mathrm{H}) .{ }^{13} \mathrm{C} \mathrm{NMR}\left(100 \mathrm{MHz}, \mathrm{CDCl}_{3}\right) \delta 31.4,33.8$, 109.7, 122.2, 128.0, 131.6, 141.6, 155.4. Anal. Calcd for $\mathrm{C}_{46} \mathrm{H}_{62} \mathrm{O}_{4}: \mathrm{C}, 81.37 ; \mathrm{H}, 9.20$; found: $\mathrm{C}$, 81.24; H, 9.27.

Bis(5-tert-butyl-2-methoxyphenyl)methanone (4a). ${ }^{13}$ TLC $R_{f} 0.82\left(50 \% \mathrm{CH}_{2} \mathrm{Cl}_{2} /\right.$ hexanes). ${ }^{1} \mathrm{H}$ NMR $\left(400 \mathrm{MHz}, \mathrm{CDCl}_{3}\right) \delta 1.25(\mathrm{~s}, 18 \mathrm{H}), 3.81(\mathrm{~s}, 6 \mathrm{H}), 3.96(\mathrm{~s}, 2 \mathrm{H}), 6.78(\mathrm{dd}, J=7.6,0.8 \mathrm{~Hz}$, 2H), 7.18 (m, 4H).

Tetrakis(2-methoxyphenyl)ethene $(2 \mathrm{~b})^{4}$ and 1,1,2,2-Tetrakis(2-methoxyphenyl)ethane (3b) ${ }^{14}$

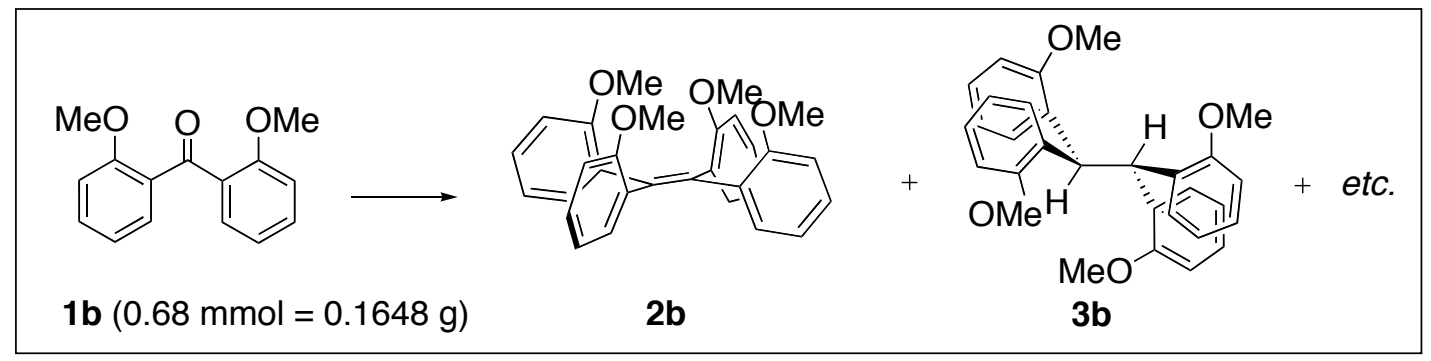


Chromatography conditions: gradient consisting of (i) 100\% hexanes (100 ml), (ii) 50\%

$\mathrm{CH}_{2} \mathrm{Cl}_{2} /$ hexanes (300 ml), (iii) $85 \% \mathrm{CH}_{2} \mathrm{Cl}_{2} /$ hexanes (200 ml), and (iv) $100 \% \mathrm{CH}_{2} \mathrm{Cl}_{2}(200 \mathrm{ml}$ ).

Tetrakis(2-methoxyphenyl)ethene (2b). ${ }^{4}$ TLC $R_{f} 0.48\left(100 \% \mathrm{CH}_{2} \mathrm{Cl}_{2}\right) .{ }^{1} \mathrm{H} \mathrm{NMR}(400 \mathrm{MHz}$, $\left.\mathrm{CDCl}_{3}\right) \delta 3.46(\mathrm{~s}, 12 \mathrm{H}), 6.66(\mathrm{~m}, 8 \mathrm{H}), 7.01$ (ddd, $\left.J=8.2,7.4,1.8 \mathrm{~Hz}, 4 \mathrm{H}\right), 7.10(\mathrm{~m}, 4 \mathrm{H})$.

1,1,2,2-Tetrakis(2-methoxyphenyl)ethane (3b). ${ }^{14}$ TLC $R_{f} 0.73\left(100 \% \mathrm{CH}_{2} \mathrm{Cl}_{2}\right) .{ }^{1} \mathrm{H}$ NMR (400 $\mathrm{MHz}, \mathrm{CDCl}_{3}$ ) $\delta 3.55$ (s, 12H), 5.77 (s, 2H), 6.57 (dd, $\left.J=8.0,1.2 \mathrm{~Hz}, 4 \mathrm{H}\right), 6.73(\operatorname{td}, J=7.6,1.2$ $\mathrm{Hz}, 4 \mathrm{H}), 6.96$ (ddd, $J=8.0,7.2,1.6 \mathrm{~Hz}, 4 \mathrm{H}), 7.33(\mathrm{dd}, J=7.6,1.6 \mathrm{~Hz}, 4 \mathrm{H})$.

\section{1,2-Bis(2'-methoxypheny)-1,2-bis(2'-methylphenyl)ethene (2c) and 1,2-bis(2'-} methoxypheny)-bis( $2^{\prime}$-methylphenyl)ethane (3c).

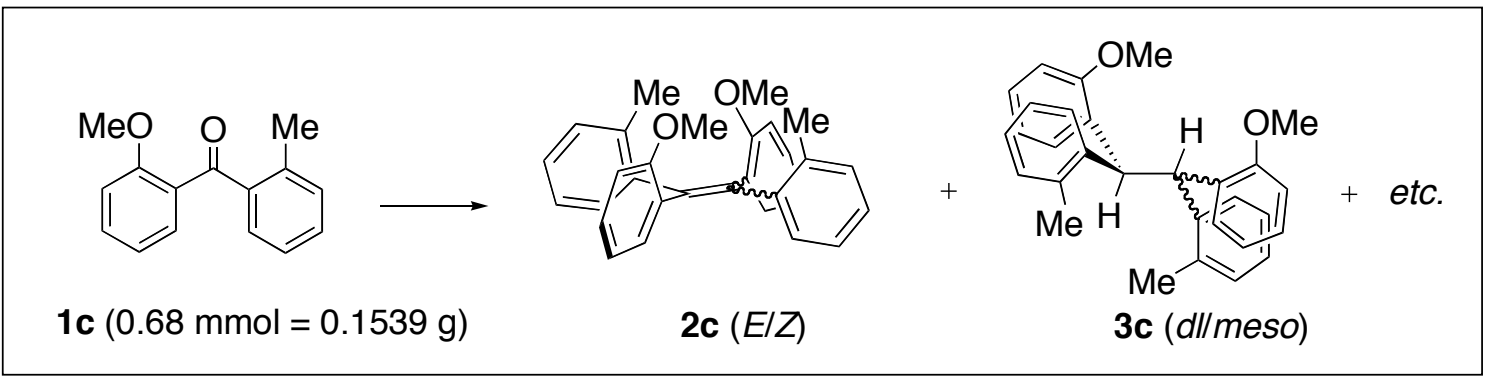

Chromatography conditions: gradient consisting of (i) 100\% hexanes (100 ml), (ii) $30 \%$ $\mathrm{CH}_{2} \mathrm{Cl}_{2} /$ hexanes (400 ml), and (iii) $50 \% \mathrm{CH}_{2} \mathrm{Cl}_{2} /$ hexanes (300 ml).

(Z/E)-1,2-Bis(2'-methoxypheny)-1,2-bis(2'-methylphenyl)ethene (2c) and (dl/meso)-1,2-bis(2'methoxypheny)-bis(2'-methylphenyl)ethane (3c). Inseparable mixture. The isomers of $\mathbf{2 c}$ and $\mathbf{3 c}$ could not be separated into pure compounds by flash column chromatography of the crude product. The identities of these compounds were determined tentatively by high resolution mass spectroscopy and by comparison with the ${ }^{1} \mathrm{H}$ NMR and ${ }^{13} \mathrm{C}$ NMR spectra of structurally similar compounds $\mathbf{2 d}, \mathbf{3 d}, \mathbf{2 h}$ and $3 \mathbf{h}$. 
(Z/E)-1,2-Bis(2'-methoxypheny)-1,2-bis(2'-methylphenyl)ethene (2c): TLC $R_{f} 0.60$ (50\%

$\mathrm{CH}_{2} \mathrm{Cl}_{2}$ /hexanes); (dl/meso)-1,2-bis(2'-methoxypheny)-bis(2'-methylphenyl)ethane (3c): TLC $R_{f}$ 0.61 (50\% $\mathrm{CH}_{2} \mathrm{Cl}_{2} /$ hexanes). ${ }^{1} \mathrm{H} \mathrm{NMR}\left(400 \mathrm{MHz}, \mathrm{CD}_{2} \mathrm{Cl}_{2}\right) \delta 2.15$ (br s, $12 \mathrm{H},(Z)-$ and $(E)-\mathrm{CH}_{3}$ of 2c), 2.21 (s, 6H, $\mathrm{CH}_{3}$ of 3c), 2.23 (s, 6H, $\mathrm{CH}_{3}$ of 3c), 3.48 (br s, 12H, (Z)- and $(E)-\mathrm{CH}_{3} \mathrm{O}$ of 2c), $3.56\left(\mathrm{~s}, 6 \mathrm{H}, \mathrm{CH}_{3} \mathrm{O}\right.$ of 3c), $3.60\left(\mathrm{~s}, 6 \mathrm{H}, \mathrm{CH}_{3} \mathrm{O}\right.$ of 3c), $5.59\left(\mathrm{~s}, 4 \mathrm{H}, \mathrm{Ar}_{2} \mathrm{CH}\right.$ of 3c), $6.59 \sim 7.46(\mathrm{~m}$, 32H, Ar-H of 2c and 3c). ${ }^{13} \mathrm{C}$ NMR (100 MHz, $\left.\mathrm{CD}_{2} \mathrm{Cl}_{2}\right) \delta$ 19.7, 19.8, 20.2 (br), 55.2 (br), 55.4, 110.1, 110.4, 111.0 (br), 111.3, 119.8 (br), 120.1, 120.3, 124.7 (br), 125.3, 125.6, 125.7, 125.9, 126.4, 126.6 (br), 126.9, 127.2, 128.1, 128.2 (br), 128.5, 129.1, 129.4, 129.8, 129.9, 130.0 (br), 130.3, 131.7 (br), 131.9, 132.3, 132.9 (br), 137.2, 137.4, 139.0 (br), 142.0, 142.4, 142.6 (br),157.1, 157.3 (br). HRMS (EI) m/z calcd for $\mathrm{C}_{30} \mathrm{H}_{28} \mathrm{O}_{2}$ (2c): 420.2084 ; found: 420.2089 (100\%), $\mathrm{C}_{30} \mathrm{H}_{30} \mathrm{O}_{2}$ (3c): 422.2208; found: $211.1123\left(\mathrm{M}^{+} / 2\right)(100 \%), 105$ (95). Copies of the ${ }^{1} \mathrm{H}$ and ${ }^{13} \mathrm{C}$ NMR spectra are provided.

(dl/meso)-1,2-Bis(2'-methoxypheny)-bis(2'-methylphenyl)ethane (3c). A portion of the crude product mixture consisting of $87 \% \mathbf{3 c}$ and $13 \% \mathbf{2 c}$ was obtained after repeated purifications by flash column chromatography. TLC $R_{f} 0.61\left(50 \% \mathrm{CH}_{2} \mathrm{Cl}_{2} /\right.$ hexanes). ${ }^{1} \mathrm{H} \mathrm{NMR}(400 \mathrm{MHz}$, $\left.\mathrm{CDCl}_{3}\right) \delta 2.15(\mathrm{~s}, 6 \mathrm{H}), 2.18(\mathrm{~s}, 6 \mathrm{H}), 3.47(\mathrm{~s}, 6 \mathrm{H}), 3.53(\mathrm{~s}, 6 \mathrm{H}), 5.52(\mathrm{~s}, 2 \mathrm{H}), 5.53(\mathrm{~s}, 2 \mathrm{H}), 6.52(\mathrm{dd}$, $J=8.4,1.2 \mathrm{~Hz}, 2 \mathrm{H}), 6.74(\mathrm{dt}, J=7.6,1.2 \mathrm{~Hz}, 4 \mathrm{H}), 6.88 \sim 7.02(\mathrm{~m}, 18 \mathrm{H}), 7.20(\mathrm{dd}, J=7.6,1.2$ $\mathrm{Hz}, 4 \mathrm{H}), 7.35(\mathrm{~d}, J=7.6 \mathrm{~Hz}, 4 \mathrm{H})$.

[2-(2-Methylphenyl)(2-methoxyphenyl)methylphenyl][2-methoxyphenyl]methanone (5). TLC $R_{f}$ 0.32 (50\% $\mathrm{CH}_{2} \mathrm{Cl}_{2} /$ hexanes). ${ }^{1} \mathrm{H}$ NMR (400 MHz, $\left.\mathrm{CDCl}_{3}\right) \delta 1.90$ (s, 3H), 2.28 (s, 3H), 3.63 (s, $3 \mathrm{H}), 6.27(\mathrm{~s}, 1 \mathrm{H}), 6.72(\mathrm{dd}, J=7.6,2.0 \mathrm{~Hz}, 1 \mathrm{H}), 6.78(\mathrm{~m}, 2 \mathrm{H}), 6.83(\mathrm{dd}, J=7.6,1.2 \mathrm{~Hz}, 1 \mathrm{H})$, $6.96(\mathrm{dd}, J=7.6,1.2 \mathrm{~Hz}, 1 \mathrm{H}), 6.83(\mathrm{t}, J=7.2 \mathrm{~Hz}, 1 \mathrm{H}), 7.08(\mathrm{~m}, 3 \mathrm{H}), 7.17(\mathrm{~m}, 3 \mathrm{H}), 7.24(\mathrm{~m}, 2 \mathrm{H})$, 7.37 (m, 2H). ${ }^{13} \mathrm{C}$ NMR (100 MHz, $\left.\mathrm{CDCl}_{3}\right) \delta$ 19.4, 20.6, 43.2, 55.3, 110.3, 120.2, 124.9, 125.4, $126.0,126.2,127.5,129.3,129.9,130.1,130.2,130.3,131.0,131.1,131.2,131.4,137.0,138.0$, 138.8, 140.5, 141.6, 142.2, 157.0, 200.4. IR $\left(\mathrm{CH}_{2} \mathrm{Cl}_{2}\right): 1662 \mathrm{~cm}^{-1}(\mathrm{C}=\mathrm{O})$. HRMS (EI) m/z calcd 
for $\mathrm{C}_{29} \mathrm{H}_{26} \mathrm{O}_{2}$ : 406.1942; found: 406.1933 (93\%), 287 (100), 119 (73), 105 (53), 91 (59). The quantity of isolated compound 5 was insufficient for elemental analysis; copies of ${ }^{1} \mathrm{H}$ and ${ }^{13} \mathrm{C}$ NMR spectra are provided.

9,10-Bis(o-tolyl)anthracene (6). ${ }^{15}$ TLC $R_{f} 0.80\left(50 \% \mathrm{CH}_{2} \mathrm{Cl}_{2} /\right.$ hexanes), ${ }^{1} \mathrm{H}$ NMR $(400 \mathrm{MHz}$, $\left.\mathrm{CDCl}_{3}\right) \delta 1.93(\mathrm{~s}, 3 \mathrm{H}), 1.94(\mathrm{~s}, 3 \mathrm{H}), 7.31 \sim 7.58(\mathrm{~m}, 16 \mathrm{H}) .{ }^{13} \mathrm{C} \mathrm{NMR}\left(100 \mathrm{MHz}, \mathrm{CDCl}_{3}\right) \delta 19.8$, $125.1,125.8,126.8,127.8,129.7,130.0,131.3,136.3,137.9,138.0,138.5$. The structure of this known compound was confirmed by X-ray crystallography (vide infra).

Tetrakis(2-methylphenyl)ethene $(2 \mathrm{~d})^{16}$ and 1,1,2,2-tetrakis(2-methylphenyl)ethane $(3 \mathrm{~d}){ }^{16}$

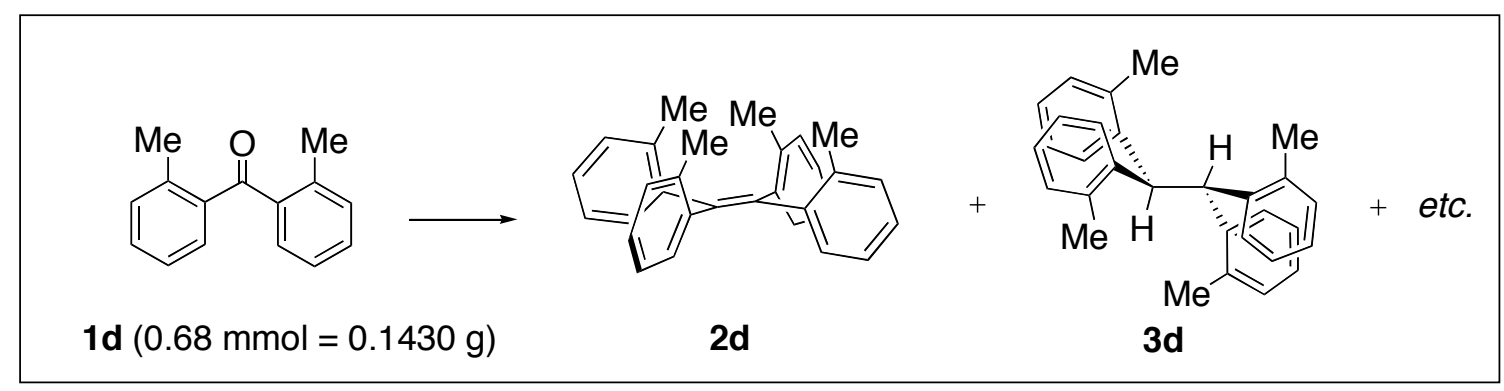

Chromatography conditions: gradient consisting of (i) $100 \%$ hexanes (100 ml), (ii) $10 \%$ $\mathrm{CH}_{2} \mathrm{Cl}_{2} /$ hexanes (300 ml), (iii) $20 \% \mathrm{CH}_{2} \mathrm{Cl}_{2} /$ hexanes $(200 \mathrm{ml})$.

Tetrakis(2-methylphenyl)ethene (2d). ${ }^{16}$ TLC $R_{f} 0.46\left(20 \% \mathrm{CH}_{2} \mathrm{Cl}_{2} /\right.$ hexanes). ${ }^{1} \mathrm{H}$ NMR (400 $\left.\mathrm{MHz}, \mathrm{CDCl}_{3}\right) \delta 2.01$ (br s, 12H), $6.88 \sim 7.04$ (br m, 16H).

1,1,2,2-Tetrakis(2-methylphenyl)ethane (3d). ${ }^{16}$ TLC $R_{f} 0.36\left(20 \% \mathrm{CH}_{2} \mathrm{Cl}_{2} /\right.$ hexanes). ${ }^{1} \mathrm{H} \mathrm{NMR}$ $\left(400 \mathrm{MHz}, \mathrm{CDCl}_{3}\right) \delta 1.92(\mathrm{~s}, 12 \mathrm{H}), 5.08(\mathrm{~s}, 2 \mathrm{H}), 6.91 \sim 7.00(\mathrm{~m}, 12 \mathrm{H}), 7.19(\mathrm{~m}, 4 \mathrm{H})$. 
Tetrakis(4-methoxy-2-methylphenyl)ethene (2e) and 1,1,2,2-Tetrakis(4-methoxy-2methylphenyl)ethane (3e)

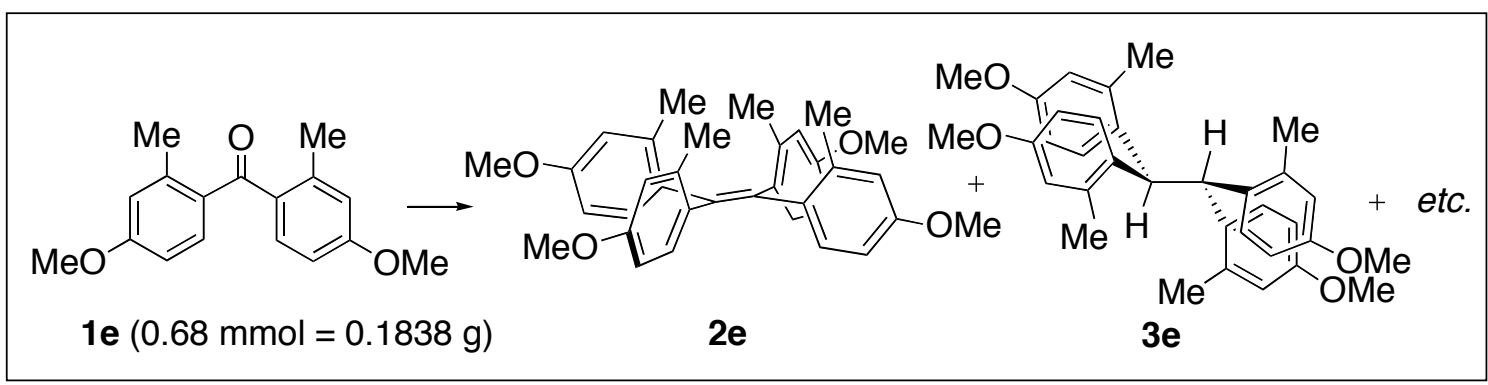

Chromatography conditions: gradient consisting of (i) $70 \% \mathrm{CH}_{2} \mathrm{Cl}_{2} /$ hexanes (500 ml) and (ii) $100 \% \mathrm{CH}_{2} \mathrm{Cl}_{2}(100 \mathrm{ml})$.

Tetrakis(4-methoxy-2-methylphenyl)ethene (2e). TLC $R_{f} 0.74\left(100 \% \mathrm{CH}_{2} \mathrm{Cl}_{2}\right) .{ }^{1} \mathrm{H} \mathrm{NMR}(400$ $\mathrm{MHz}, \mathrm{CDCl}_{3}$ ) $\delta 1.96$ (br s, 12H), 3.71 (s, 12H), 6.47 (br m, 8H), $6.88 \sim 6.91$ (br m, 4H). ${ }^{1} \mathrm{H}$ NMR (400 MHz, toluene- $\left.\mathrm{d}_{8}, 80{ }^{\circ} \mathrm{C}\right) \delta 2.04(\mathrm{~s}, 12 \mathrm{H}), 3.30(\mathrm{~s}, 12 \mathrm{H}), 6.43(\mathrm{dd}, J=8.4,2.4 \mathrm{~Hz}, 4 \mathrm{H})$, $6.51(\mathrm{~d}, J=2.8 \mathrm{~Hz}, 4 \mathrm{H}), 7.04(\mathrm{~d}, J=8.0 \mathrm{~Hz}, 4 \mathrm{H}) .{ }^{13} \mathrm{C}$ NMR $\left(100.1 \mathrm{MHz}, \mathrm{Tol}-\mathrm{d}_{8}, 100{ }^{\circ} \mathrm{C}\right) \delta 21.2$, 54.8, 111.4, 116.3, 133.8, 136.4, 137.5, 138.3, 159.0. HRMS (EI) m/z calcd for $\mathrm{C}_{34} \mathrm{H}_{36} \mathrm{O}_{4}$ : 508.2616; found: 508.2614 (100\%). Anal. Calcd for $\mathrm{C}_{34} \mathrm{H}_{36} \mathrm{O}_{4}: \mathrm{C}, 80.29 ; \mathrm{H}, 7.13$; found: C, 80.08; H, 7.15 .

1,1,2,2-Tetrakis(4-methoxy-2-methylphenyl)ethane (3e). TLC $R_{f} 0.67\left(100 \% \mathrm{CH}_{2} \mathrm{Cl}_{2}\right) .{ }^{1} \mathrm{H} \mathrm{NMR}$ $\left(400 \mathrm{MHz}, \mathrm{CDCl}_{3}\right) \delta 1.91(\mathrm{~s}, 12 \mathrm{H}), 3.71(\mathrm{~s}, 12 \mathrm{H}), 4.86(\mathrm{~s}, 2 \mathrm{H}), 6.43(\mathrm{~d}, J=2.8 \mathrm{~Hz}, 4 \mathrm{H}), 6.55$ $(\mathrm{dd}, J=8.8,2.8 \mathrm{~Hz}, 4 \mathrm{H}), 7.05(\mathrm{~d}, J=8.4 \mathrm{~Hz}, 4 \mathrm{H}) .{ }^{13} \mathrm{C} \mathrm{NMR}\left(100 \mathrm{MHz}, \mathrm{CDCl}_{3}\right) \delta 19.7,54.9$, $110.6,115.3,130.5,134.0,137.8,157.1$. The quantity of isolated $\mathbf{3 e}$ was insufficient for elemental analysis; copies of ${ }^{1} \mathrm{H}$ and ${ }^{13} \mathrm{C}$ NMR spectra are provided. The structure of $\mathbf{3 e}$ was confirmed by X-ray crystallography (vide infra). 
Spectroscopic and analytical data for the compounds reported in Table 3. For known compounds referenced to the literature, only partial and/or updated data are included.

1,2-Bis(4'-methoxypheny)-1,2-bis(2'-methoxyphenyl)ethene (2f) and $d l$ - and meso-1,2-bis(4'methoxypheny)-1,2-bis(2'-methoxyphenyl)ethane (3f).

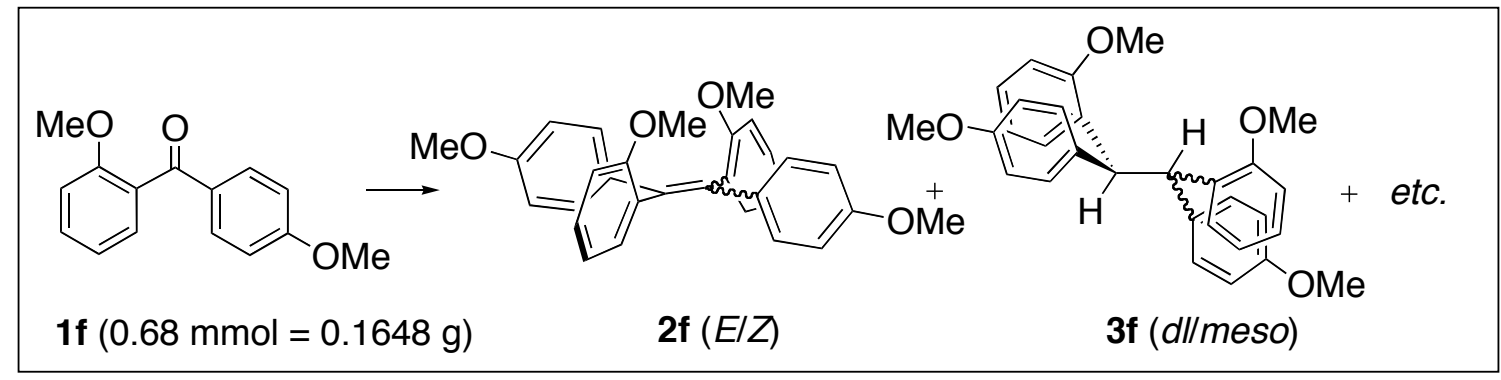

Chromatography conditions: gradient consisting of (i) 100\% hexanes (100 ml), (ii) 80\% $\mathrm{CH}_{2} \mathrm{Cl}_{2} /$ hexanes $(300 \mathrm{ml})$, (iii) $85 \% \mathrm{CH}_{2} \mathrm{Cl}_{2} /$ hexanes $(300 \mathrm{ml})$, and (iv) $100 \% \mathrm{CH}_{2} \mathrm{Cl}_{2}(100 \mathrm{ml})$.

(Z)-1,2-Bis(4'-methoxypheny)-1,2-bis(2'-methoxyphenyl)ethene (2f). Major isomer. A portion of this isomer of $\mathbf{2} \mathbf{f}$ was obtained in a pure state after flash column chromatography of the crude product. The stereochemistry was tentatively assigned based on spectroscopic comparisons to $(Z)$ - and $(E)$-2f - specifically, the relatively broad signal of the ortho-methoxy functionality in the ${ }^{1} \mathrm{H}$ NMR spectra. TLC $R_{f} 0.50\left(100 \% \mathrm{CH}_{2} \mathrm{Cl}_{2}\right)$. ${ }^{1} \mathrm{H} \mathrm{NMR}\left(400 \mathrm{MHz}, \mathrm{CDCl}_{3}\right) \delta 3.50$ (br s, $6 \mathrm{H}), 3.75(\mathrm{~s}, 6 \mathrm{H}), 6.62 \sim 6.71(\mathrm{~m}, 8 \mathrm{H}), 7.00(\mathrm{~m}, 4 \mathrm{H}), 7.06(\mathrm{dt}, J=8.4,1.6 \mathrm{~Hz}, 4 \mathrm{H}) .{ }^{13} \mathrm{C} \mathrm{NMR}$ $\left(100 \mathrm{MHz}, \mathrm{CDCl}_{3}\right) \delta 55.0,55.4,111.1,112.7,119.8,127.7,131.5,133.7,136.8,157.2,157.6$. HRMS (EI) m/z calcd for $\mathrm{C}_{30} \mathrm{H}_{28} \mathrm{O}_{4}$ : 452.1989; found: 452.2000 (100\%), 121 (21). Anal. Calcd for $\mathrm{C}_{30} \mathrm{H}_{28} \mathrm{O}_{4}$ : C, 79.62; H, 6.24; found: C, 79.77; H, 6.30.

(E)-1,2-Bis(4'-methoxypheny)-1,2-bis(2'-methoxyphenyl)ethene (2f). Minor isomer. This isomer of $2 \mathbf{f}$ was obtained as a mixture with $(Z)$-isomer after flash column chromatography of the crude product. The spectroscopic data were based on comparison of those of a mixture of $(Z)$ - and $(E)$ isomers with those of pure (Z)-isomer. TLC $R_{f} 0.48\left(100 \% \mathrm{CH}_{2} \mathrm{Cl}_{2}\right) .{ }^{1} \mathrm{H}$ NMR $(400 \mathrm{MHz}$, 
$\left.\mathrm{CDCl}_{3}\right) \delta 3.53($ br s, $6 \mathrm{H}), 3.71(\mathrm{~s}, 6 \mathrm{H}), 6.61(\mathrm{dd}, J=6.8,2.0 \mathrm{~Hz}, 4 \mathrm{H}), 6.81(\mathrm{~m}, 4 \mathrm{H}), 6.98(\mathrm{dd}, J=$ 6.8, $2.0 \mathrm{~Hz}, 4 \mathrm{H}), 7.18(\mathrm{~m}, 4 \mathrm{H}) .{ }^{13} \mathrm{C} \mathrm{NMR}\left(100 \mathrm{MHz}, \mathrm{CDCl}_{3}\right) \delta$ 54.9, 55.5, 111.5, 112.3, 120.2, 127.8, 130.5, 132.5, 133.2, 136.3, 157.1, 157.4. HRMS (EI) m/z calcd for $\mathrm{C}_{30} \mathrm{H}_{28} \mathrm{O}_{4}$ : 452.1989; found: 452.2000 (100\%), 121 (21). Anal. Calcd for $\mathrm{C}_{30} \mathrm{H}_{28} \mathrm{O}_{4}$ : C, 79.62; H, 6.24; found: C, 79.77; H, 6.30.

(dl/meso)-1,2-Bis(4'-methoxypheny)-1,2-bis(2'-methoxyphenyl)ethane (3f). These diastereoisomers of $\mathbf{3 f}$ were obtained as an approximate 1:1 mixture after flash column chromatography of the crude product. TLC $R_{f} 0.58\left(100 \% \mathrm{CH}_{2} \mathrm{Cl}_{2}\right) .{ }^{1} \mathrm{H} \mathrm{NMR}(400 \mathrm{MHz}$, $\left.\mathrm{CDCl}_{3}\right) \delta 3.67(\mathrm{~s}, 6 \mathrm{H}), 3.69(\mathrm{~s}, 6 \mathrm{H}), 3.70(\mathrm{~s}, 6 \mathrm{H}), 3.74(\mathrm{~s}, 6 \mathrm{H}), 5.27(\mathrm{~s}, 2 \mathrm{H}), 5.37(\mathrm{~s}, 2 \mathrm{H}), 6.60 \sim$ $6.65(\mathrm{~m}, 12 \mathrm{H}), 6.76(\mathrm{dt}, J=7.6,1.6 \mathrm{~Hz}, 2 \mathrm{H}), 6.81(\mathrm{dt} J=7.6,1.2 \mathrm{~Hz}, 2 \mathrm{H}), 6.98(\mathrm{dd}, J=8.0,1.0$ $\mathrm{Hz}, 2 \mathrm{H}), 7.02(\mathrm{dd}, J=8.0,1.0 \mathrm{~Hz}, 2 \mathrm{H}), 7.13(\mathrm{ddd}, J=8.4,6.8,2.0 \mathrm{~Hz}, 8 \mathrm{H}), 7.32(\mathrm{dd}, J=7.6,1.6$ $\mathrm{Hz}, 2 \mathrm{H}), 7.37(\mathrm{dd}, J=7.6,1.6 \mathrm{~Hz}, 2 \mathrm{H}) .{ }^{13} \mathrm{C} \mathrm{NMR}\left(100 \mathrm{MHz}, \mathrm{CDCl}_{3}\right) \delta 55.0,55.5,110.5,110.7$, $112.9,113.1,120.2,120.4,126.4,126.5,127.9,128.4,129.5,129.7,132.8,133.1,135.9,136.0$, 156.5, 156.6, 157.1, 157.2. HRMS (EI) m/z calcd for $\mathrm{C}_{30} \mathrm{H}_{30} \mathrm{O}_{4}: 454.2144$; found: 454.2139 (0.72\%), 227 (100), 121 (77). The quantity of purified 3f was insufficient to obtain elemental analysis; for this reason, ${ }^{1} \mathrm{H}$ and ${ }^{13} \mathrm{C}$ NMR spectra are provided (vide infra).

\section{1,2-Bis $\left(2^{\prime}, 4^{\prime}\right.$-dimethoxypheny)-1,2-bis(phenyl)ethene (2g) and 1,2-bis $\left(2^{\prime}, 4^{\prime}\right.$ -} dimethoxyphenyl)-1,2-bis(phenyl)ethane (3g)

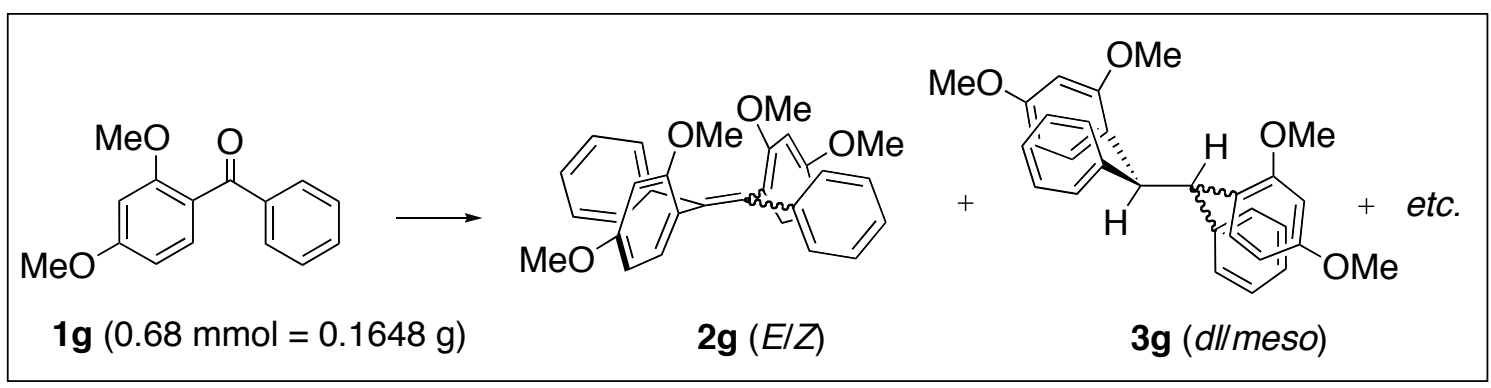


Chromatography conditions: gradient consisting of (i) $50 \% \mathrm{CH}_{2} \mathrm{Cl}_{2} /$ hexanes (300 ml), (ii) $60 \%$ $\mathrm{CH}_{2} \mathrm{Cl}_{2} /$ hexanes (200 ml), and (iii) 70\% $\mathrm{CH}_{2} \mathrm{Cl}_{2} /$ hexanes (200 ml).

(Z)-1,2-Bis(2', 4'-dimethoxyphenyl)-1,2-diphenylethene (2g). Major isomer. A portion of this isomer of $\mathbf{2 g}$ was obtained in a pure state by recrystallization of the mixture of $(Z)$ - and $(E)$ isomers from $\mathrm{CH}_{2} \mathrm{Cl}_{2} /$ pentane after flash column chromatography of the crude product. The stereochemistry was tentatively assigned based on spectroscopic comparisons to (Z)- and (E)-2h - specifically, the relatively broad signal and chemical shift of ortho-methoxy functionality in the ${ }^{1} \mathrm{H}$ NMR spectrum. TLC $R_{f} 0.25\left(50 \% \mathrm{CH}_{2} \mathrm{Cl}_{2} /\right.$ hexanes). ${ }^{1} \mathrm{H}$ NMR (400 $\left.\mathrm{MHz}, \mathrm{CDCl}_{3}\right) \delta 3.47$ (br s, 6H), $3.74(\mathrm{~s}, 6 \mathrm{H}), 6.28(\mathrm{~m}, 4 \mathrm{H}), 6.97(\mathrm{~d}, J=8.0 \mathrm{~Hz}, 2 \mathrm{H}), 7.05(\mathrm{~m}, 10 \mathrm{H}) .{ }^{13} \mathrm{C}$ NMR $(100$ $\left.\mathrm{MHz}, \mathrm{CDCl}_{3}\right) \delta$ 55.1, 55.4, 98.8,103.9, 125.6, 126.5, 127.1, 130.5, 131.9, 137.7, 143.2, 158.1, 159.6. HRMS (EI) m/z calcd for $\mathrm{C}_{30} \mathrm{H}_{28} \mathrm{O}_{4}: 452.1989$; found: 452.1988 (100\%). Anal. Calcd for $\mathrm{C}_{30} \mathrm{H}_{28} \mathrm{O}_{4}$ : C, 79.62; H, 6.24; found: C, 79.08; H, 6.47. To confirm purity despite the poor elemental analysis, copies of the ${ }^{1} \mathrm{H}$ and ${ }^{13} \mathrm{C}$ NMR spectra are provided.

(Z/E)-1,2-Bis(2', 4'-dimethoxypheny)-1,2-diphenylethene (2g). Mixture of stereoisomers. The isomers of $2 \mathbf{g}$ were obtained in an $Z: E$ ratio of approximately $8: 1$ after flash column chromatography of the crude product. The minor $(E)$-isomer could not be isolated in a pure state. TLC $R_{f} 0.25$ (50\% $\mathrm{CH}_{2} \mathrm{Cl}_{2} /$ hexanes). ${ }^{1} \mathrm{H}$ NMR (400 MHz, $\left.\mathrm{CDCl}_{3}\right) \delta 3.43$ (s, $6 \mathrm{H},(E)$ $\mathrm{CH}_{3} \mathrm{O}$ ), 3.47 (br s, 6H, $(\mathrm{Z})-\mathrm{CH}_{3} \mathrm{O}$ ), 3.74 (s, 6H, $\left.(\mathrm{Z})-\mathrm{CH}_{3} \mathrm{O}\right), 3.74$ (s, 6H, $\left.(E)-\mathrm{CH}_{3} \mathrm{O}\right), 6.27 \sim 6.33$ $(\mathrm{m}, 8 \mathrm{H}), 6.96 \sim 7.08(\mathrm{~m}, 24 \mathrm{H}) .{ }^{13} \mathrm{C} \mathrm{NMR}\left(100 \mathrm{MHz}, \mathrm{CDCl}_{3}\right) \delta 55.1$ (overlapping $\mathrm{CH}_{3} \mathrm{O}$ signals of (Z)- and (E)- isomers), 55.4 (overlapping $\mathrm{CH}_{3} \mathrm{O}$ signals of (Z)- and $(E)$ - isomers), 98.8, 99.0, 103.9, 104.2, 125.5, 125.6, 126.5, 126.9, 127.1, 130.5, 131.9, 133.1, 137.7, 143.2, 144.2, 158.1, 158.8, 159.6, 160.1. HRMS (EI) m/z calcd for $\mathrm{C}_{30} \mathrm{H}_{28} \mathrm{O}_{4}$ : 452.1989; found: 452.1988 (100\%). Anal. Calcd for $\mathrm{C}_{30} \mathrm{H}_{28} \mathrm{O}_{4}$ : C, 79.62; H, 6.24; found: C, 78.92; H, 6.13. To confirm purity despite the unsuccessful elemental analysis, copies of the ${ }^{1} \mathrm{H}$ and ${ }^{13} \mathrm{C}$ NMR spectra are provided. 
(dl/meso)-1,2-Bis(2',4'-dimethoxyphenyl)-1,2-diphenylethane (3g). These diastereoisomers of 3g were obtained as an approximately $1: 1$ mixture after flash column chromatography of the crude product. TLC $R_{f} 0.30\left(50 \% \mathrm{CH}_{2} \mathrm{Cl}_{2} /\right.$ hexanes). ${ }^{1} \mathrm{H}$ NMR (400 MHz, $\left.\mathrm{CDCl}_{3}\right) \delta 3.63(\mathrm{~s}, 6 \mathrm{H})$, 3.69 (s, 6H), 3.70 (s, 6H), $3.71(\mathrm{~s}, 6 \mathrm{H}), 5.17$ (s, 2H), $5.27(\mathrm{~s}, 2 \mathrm{H}), 6.22(\mathrm{~d}, J=2.4 \mathrm{~Hz}, 2 \mathrm{H}), 6.27$ $(\mathrm{d}, J=2.4 \mathrm{~Hz}, 2 \mathrm{H}), 6.30(\mathrm{t}, J=2.4 \mathrm{~Hz}, 2 \mathrm{H}), 6.33(\mathrm{t}, J=2.4 \mathrm{~Hz}, 2 \mathrm{H}), 6.91 \sim 6.99(\mathrm{~m}, 4 \mathrm{H}), 7.05$ (m, 8H), $7.11 \sim 7.25(\mathrm{~m}, 12 \mathrm{H}) .{ }^{13} \mathrm{C} \mathrm{NMR}\left(100 \mathrm{MHz}, \mathrm{CDCl}_{3}\right)$ 855.1, 55.4, 55.5, 104.1, 104.3, $125.0,125.1,125.2,125.3,127.4,127.5,128.3,128.4,128.6,128.7,128.8,128.9,144.1,157.5$, 157.7, 158.5. HRMS (EI) m/z calcd for $\mathrm{C}_{30} \mathrm{H}_{30} \mathrm{O}_{4}$ : 454.2144; found: 454.2122 (1.61\%), 227 (100), 91 (74). The quantity of purified $\mathbf{3 g}$ was insufficient to obtain elemental analysis; copies of ${ }^{1} \mathrm{H}$ and ${ }^{13} \mathrm{C}$ NMR spectra are provided.

\section{1,2-Bis(2'-methoxyphenyl)-1,2-diphenylethene $(2 \mathrm{~h})^{17}$ and 1,2-bis(2'-methoxyphenyl)-1,2- diphenylethane $(3 h)$}

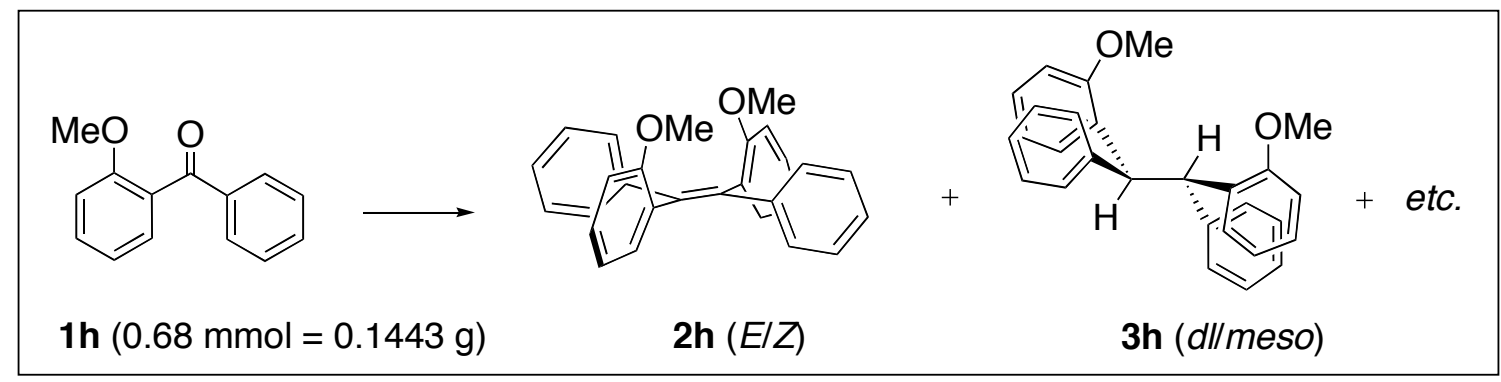

Chromatography conditions: gradient consisting of (i) 100\% hexanes (100 ml), (ii) 30\% $\mathrm{CH}_{2} \mathrm{Cl}_{2} /$ hexanes $(500 \mathrm{ml})$, and (iii) $50 \% \mathrm{CH}_{2} \mathrm{Cl}_{2} /$ hexanes $(100 \mathrm{ml})$.

(Z)-1,2-Bis(2'-methoxyphenyl)-1,2-diphenylethene (2h). ${ }^{17}$ Major isomer. A portion of this isomer of $\mathbf{2 h}$ was obtained in a pure state after flash column chromatography of the crude product. The stereochemistry was confirmed by X-ray crystallography (vide infra). TLC $R_{f} 0.54$ 
(50\% $\mathrm{CH}_{2} \mathrm{Cl}_{2} /$ hexanes). ${ }^{1} \mathrm{H} \mathrm{NMR}\left(400 \mathrm{MHz}, \mathrm{CDCl}_{3}\right) \delta 3.48(\mathrm{~s}, 6 \mathrm{H}), 6.69(\mathrm{~m}, 4 \mathrm{H}), 7.06$ (m, 14H). HRMS (EI) m/z calcd for $\mathrm{C}_{28} \mathrm{H}_{24} \mathrm{O}_{2}$ : 392.1776; found: 392.1775 (100\%).

(E)-1,2-Bis(2'-methoxyphenyl)-1,2-diphenylethene (2h). ${ }^{17}$ Minor isomer. The (E)-isomer of $\mathbf{2 h}$ was obtained as a mixture with the $(Z)$-isomer after flash column chromatography of the crude product. The spectroscopic assignments were based on comparison of those of a mixture of (Z)and $(E)$ - isomers with those of pure (Z)-isomer. TLC $R_{f} 0.53\left(50 \% \mathrm{CH}_{2} \mathrm{Cl}_{2} /\right.$ hexanes). ${ }^{1} \mathrm{H}$ NMR $\left(400 \mathrm{MHz}, \mathrm{CDCl}_{3}\right) \delta 3.46(\mathrm{~s}, 6 \mathrm{H}), 6.72(\mathrm{~m}, 4 \mathrm{H}), 6.79 \sim 7.14(\mathrm{~m}, 14 \mathrm{H}) . \mathrm{HRMS}(\mathrm{EI}) \mathrm{m} / \mathrm{z}$ calcd for $\mathrm{C}_{28} \mathrm{H}_{24} \mathrm{O}_{2}: 392.1776$; found: 392.1775 (100\%).

(dl/meso)-1,2-Bis(2'-methoxyphenyl)-1,2-diphenylethane (3h). These diastereoisomers of $\mathbf{3 h}$ were obtained as an approximate 1:1 mixture after flash column chromatography of the crude product. TLC $R_{f} 0.56\left(50 \% \mathrm{CH}_{2} \mathrm{Cl}_{2} /\right.$ hexanes). ${ }^{1} \mathrm{H} \mathrm{NMR}\left(400 \mathrm{MHz}, \mathrm{CDCl}_{3}\right) \delta 3.68(\mathrm{~s}, 6 \mathrm{H}), 3.74$ $(\mathrm{s}, 6 \mathrm{H}), 5.36(\mathrm{~s}, 2 \mathrm{H}), 5.46(\mathrm{~s}, 2 \mathrm{H}), 6.68(\mathrm{dt}, J=9.2,0.8 \mathrm{~Hz}, 4 \mathrm{H}), 6.79(\mathrm{~m}, 4 \mathrm{H}), 6.96 \sim 7.10(\mathrm{~m}$, $16 \mathrm{H}), 7.24(\mathrm{~m}, 8 \mathrm{H}), 7.36(\mathrm{dd}, J=7.6,1.6 \mathrm{~Hz}, 2 \mathrm{H}), 7.40(\mathrm{dd}, J=7.6,1.6 \mathrm{~Hz}, 2 \mathrm{H}) .{ }^{13} \mathrm{C} \mathrm{NMR}(100$ $\left.\mathrm{MHz}, \mathrm{CDCl}_{3}\right) \delta 55.5,110.5,110.6,120.2,120.4,125.3,125.4,126.6,126.7,127.5,127.6,128.1$, 128.6, 128.7, 128.9, 132.3, 132.6, 143.6, 143.7, 156.7, 156.8. HRMS (EI) m/z calcd for $\mathrm{C}_{28} \mathrm{H}_{26} \mathrm{O}_{2}: 394.1933$; found: 394.1923 (2.30\%), 197 (90), 165 (23), 91(100). Anal. Calcd for $\mathrm{C}_{28} \mathrm{H}_{26} \mathrm{O}_{2}:$ C, 85.25; H, 6.64; found: C, 85.63; H, 6.73.

\section{1,2-Bis(2'-methoxypheny)-1,2-bis[4'-(trifluoromethyl)phenyl]ethene (2i).}

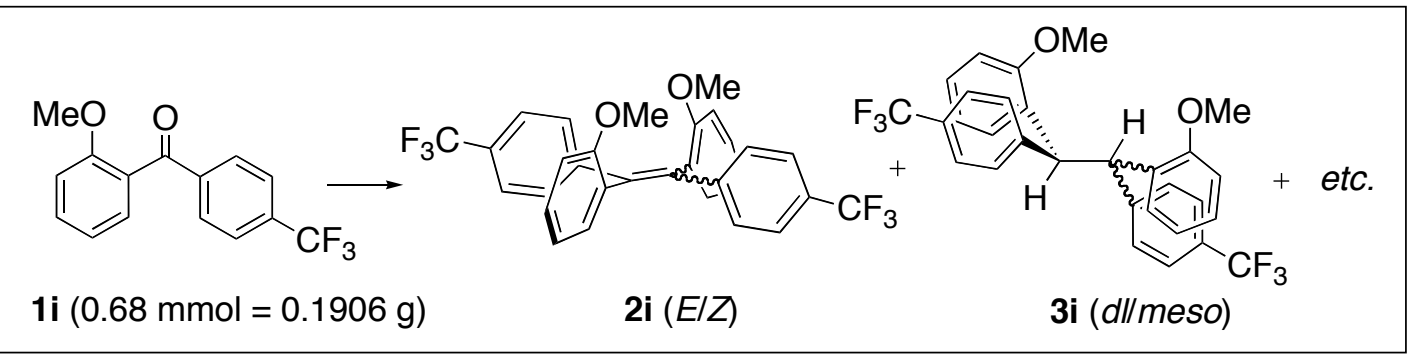


Chromatography conditions: gradient consisting of (i) 100\% hexanes (100 ml), (ii) 20\% $\mathrm{CH}_{2} \mathrm{Cl}_{2} /$ hexanes $(700 \mathrm{ml})$, and (iii) $100 \% \mathrm{CH}_{2} \mathrm{Cl}_{2}(200 \mathrm{ml})$.

(2-Methoxyphenyl)[4-(trifuoromethyl)phenyl]methanone (1i). ${ }^{18}$ This compound is nominally known, but no spectroscopic data have been previously reported. TLC $R_{f} 0.67$ (70\% $\mathrm{CH}_{2} \mathrm{Cl}_{2}$ /hexanes). ${ }^{1} \mathrm{H} \mathrm{NMR}\left(400 \mathrm{MHz}, \mathrm{CDCl}_{3}\right) \delta 3.68(\mathrm{~s}, 3 \mathrm{H}), 3.66(\mathrm{~s}, 12 \mathrm{H}), 6.99(\mathrm{~d}, J=8.4 \mathrm{~Hz}$, 1H), $7.06(\mathrm{td}, J=7.6,0.8 \mathrm{~Hz}, 1 \mathrm{H}), 7.41(\mathrm{dd}, J=7.6,1.6 \mathrm{~Hz}, 1 \mathrm{H}), 7.50$ (ddd, $J=8.4,7.6,1.6 \mathrm{~Hz}$, 1H), $7.68(\mathrm{~d}, J=8.4 \mathrm{~Hz}, 2 \mathrm{H}), 7.88(\mathrm{~d}, J=8.4 \mathrm{~Hz}, 2 \mathrm{H}) .{ }^{13} \mathrm{C} \mathrm{NMR}\left(100 \mathrm{MHz}, \mathrm{CDCl}_{3}\right) \delta 55.4$, $111.4,119.6,120.7,122.3,125.0(\mathrm{q}, J=3.7 \mathrm{~Hz}), 127.9,129.7,129.9,132.7,133.8(\mathrm{q}, J=32.5$ $\mathrm{Hz})$, 140.9, 157.5, 195.3. Anal. Calcd for $\mathrm{C}_{15} \mathrm{H}_{11} \mathrm{~F}_{3} \mathrm{O}_{2}$ : C, 64.29; H, 3.96; found: C, 63.99; H, 4.04 .

(Z)-1,2-Bis(2'-methoxypheny)-1,2-bis[4'-(trifluoromethyl)phenyl]ethene (2i). Major isomer. A portion of this isomer of $\mathbf{2 i}$ was obtained in a pure state after flash column chromatography of the crude product. The stereochemistry was tentatively assigned based on spectroscopic comparisons to $(Z)$ - and $(E)$-2h - specifically, the relatively broad signal and chemical shift of the orthomethoxy functionality in the ${ }^{1} \mathrm{H}$ NMR spectrum. TLC $R_{f} 0.30\left(50 \% \mathrm{CH}_{2} \mathrm{Cl}_{2} /\right.$ hexanes $)$ and $R_{f} 0.33$ (10\% acetone/hexanes). ${ }^{1} \mathrm{H}$ NMR (400 $\left.\mathrm{MHz} \mathrm{CDCl}_{3}\right) \delta 3.50$ (br s, 6H), 6.71 (m, 4H), 7.04 (d, J $=7.2 \mathrm{~Hz}, 2 \mathrm{H}), 7.13(\mathrm{~m}, 6 \mathrm{H}), 7.34(\mathrm{~d}, J=8.4 \mathrm{~Hz}, 4 \mathrm{H}) .{ }^{13} \mathrm{C} \mathrm{NMR}\left(100 \mathrm{MHz}, \mathrm{CDCl}_{3}\right) \delta 55.2$, 111.1, 120.1, 122.9, $124.4(\mathrm{q}, J=3.7 \mathrm{~Hz}), 125.6,128.2(\mathrm{q}, J=31.9 \mathrm{~Hz}), 128.7,130.6,131.2$, 132.0, 138.7, 145.9, 157.1. HRMS (EI) m/z calcd for $\mathrm{C}_{30} \mathrm{H}_{22} \mathrm{~F}_{6} \mathrm{O}_{2}$ : 528.1531; found: 528.1524 (100\%), 57 (23). Anal. Calcd for $\mathrm{C}_{30} \mathrm{H}_{22} \mathrm{~F}_{6} \mathrm{O}_{2}$ : C, 68.18; H, 4.19; found: C, 68.24; H, 4.26.

(E)-1,2-Bis(2'-methoxypheny)-1,2-bis[4'-(trifluoromethyl)phenyl]ethene (2i). Minor isomer. A portion of this isomer of $\mathbf{2} \mathbf{i}$ was obtained in a pure state after two purifications by flash column chromatography. TLC $R_{f} 0.30\left(50 \% \mathrm{CH}_{2} \mathrm{Cl}_{2} /\right.$ hexanes $)$ and $R_{f} 0.40\left(10 \%\right.$ acetone/hexanes). ${ }^{1} \mathrm{H}$ NMR (400 MHz, $\left.\mathrm{CDCl}_{3}\right) \delta 3.48(\mathrm{~s}, 6 \mathrm{H}), 6.77(\mathrm{~m}, 4 \mathrm{H}), 7.01(\mathrm{~d}, J=7.2 \mathrm{~Hz}, 2 \mathrm{H}), 7.11(\mathrm{~d}, J=8.4$ $\mathrm{Hz}, 4 \mathrm{H}), 7.19(\mathrm{tdd}, J=8.4,1.6,0.8 \mathrm{~Hz}, 2 \mathrm{H}), 7.28(\mathrm{~d}, J=8.4 \mathrm{~Hz}, 4 \mathrm{H}) .{ }^{13} \mathrm{C} \mathrm{NMR}(100 \mathrm{MHz}$, 
$\left.\mathrm{CDCl}_{3}\right) \delta 55.3,111.4,120.5,122.9,124.0(\mathrm{q}, J=3.7 \mathrm{~Hz}), 125.6,128.0(\mathrm{q}, J=32.1 \mathrm{~Hz}), 128.9$, 129.6, 131.2, 132.4, 138.5, 146.9, 157.4. HRMS (EI) m/z calcd for $\mathrm{C}_{30} \mathrm{H}_{22} \mathrm{~F}_{6} \mathrm{O}_{2}: 528.1531$; found: $528.1524(100 \%), 57$ (23). Anal. Calcd for $\mathrm{C}_{30} \mathrm{H}_{22} \mathrm{~F}_{6} \mathrm{O}_{2}: \mathrm{C}, 68.18 ; \mathrm{H}, 4.19$; found: $\mathrm{C}$, $68.24 ; \mathrm{H}, 4.26$.

(dl/meso)-1,2-Bis(2'-methoxypheny)-1,2-bis[4'-(trifluoromethyl)phenyl]ethane (3i). The mixture of ethanes could not be isolated free of minor byproducts; no definitive spectroscopic data could be obtained.

(2-Methoxyphenyl)[4-(trifuoromethyl)phenyl]methanol. ${ }^{18}$ This minor byproduct is known, but no spectroscopic data have been reported in the literature. TLC $R_{f} 0.36\left(70 \% \mathrm{CH}_{2} \mathrm{Cl}_{2} /\right.$ hexanes). ${ }^{1} \mathrm{H}$ NMR (400 MHz, $\left.\mathrm{CDCl}_{3}\right) \delta 3.13(\mathrm{~d}, J=5.6 \mathrm{~Hz}, 1 \mathrm{H}), 3.82(\mathrm{~s}, 3 \mathrm{H}), 6.09$ (d, J=5.6 Hz, 1H), 6.91 $(\mathrm{dd}, J=8.4,1.2 \mathrm{~Hz}, 1 \mathrm{H}), 6.98(\mathrm{td}, J=7.6,0.8 \mathrm{~Hz}, 1 \mathrm{H}), 7.23(\mathrm{dd}, J=7.6,2.0 \mathrm{~Hz}, 1 \mathrm{H}), 7.30$ (ddd, $J=8.4,7.6,2.0 \mathrm{~Hz}, 1 \mathrm{H}), 7.52(\mathrm{~d}, J=8.0 \mathrm{~Hz}, 2 \mathrm{H}), 7.59(\mathrm{~d}, J=8.0 \mathrm{~Hz}, 2 \mathrm{H}) .{ }^{13} \mathrm{C}$ NMR $(100$ $\left.\mathrm{MHz}, \mathrm{CDCl}_{3}\right) \delta 55.4,110.9,120.9,122.9,125.1$ (q, $\left.J=3.7 \mathrm{~Hz}\right), 125.6,126.7,127.8,129.1$, $129.2(\mathrm{q}, J=32.1 \mathrm{~Hz}), 131.2,147.4,156.6 . \mathrm{IR}\left(\mathrm{CH}_{2} \mathrm{Cl}_{2}\right): 3387 \mathrm{~cm}^{-1}(\mathrm{OH})$. HRMS (EI) m/z calcd for $\mathrm{C}_{15} \mathrm{H}_{13} \mathrm{O}_{2} \mathrm{~F}_{3}$ : 282.0865; found: 282.0868 (100\%), 218 (20), 264 (54), 263 (29), 173 (35), 159 (21), 145 (24), 135 (31), 107 (35), 91(60).

\section{References.}

1. (a) McMurry J. E.; Fleming, M. P.; Kees, K. L.; Krepski, L. R. J. Org. Chem. 1978, 43, 3255-3266; (b) Lectka, T. in Active Metals: Preparation, Characterization, Applications, Fürstner, A., ed., VCH, New York, 1996, Chapter 3.

2. J. M. Sullivan (Boulder Scientific Company, USA) U.S. Patent 63070632001 (Oct. 23).

3. (a) Handbook of Thin-Layer Chromatography Sherman, J.; Fried, B., Eds., Marcel Dekker, New York, NY, 1991; (b) Thin-Layer Chromatography Reagents and Detection Methods, 
Vol. 1a: Physical and Chemical Detection Methods: Fundamentals, Reagents I Jork, H. H.;

Funk, W.; Fischer, W.; Wimmer, H. VHC, Weinheim, Germany, 1990.

4. Verkerk, U.; Fujita, M.; Dzwiniel, T. D.; McDonald, R.; Stryker, J. M. J. Am. Chem. Soc. 2002, 124, 9988-9989.

5. Eisenbraun, E. J. Organic Syntheses Wiley, New York, 1973, Collect. Vol. 5, 310-314.

6. Mitchell, R. H.; Lai, Y. H. J. Org. Chem. 1984, 49, 2534-2540.

7. Einhorn, J.; Einhorn, C.; Luche, J. L. Synth. Commun. 1990, 20, 1105-1112.

8. Nahm, S.; Weinreb, S. M. Tetrahedron Lett. 1981, 22, 3815-3818.

9. Harder, S.; Boersma, J.; Brandsma, L.; Kanters, J. A.; Duisenberg, A. J. M.; Van Lenthe, J. H. Organometallics 1990, 9, 511-516.

10. Bratulescu, G. Rev. Roun. Chem. 1980, 15, 239-243.

11. Eapen, K. C.; Tamborski, C. J. Fluorine Chem. 1980, 15, 239-243.

12. This so-called "instant method" was introduced by Fürstner, et al: A. Fürstner; A. Hupperts; A. Ptock; E. Jassen J. Org. Chem. 1994, 59, 5215.

13. Yamato, T.; Inoue, H.; Maeda, K.; Tashiro, M. J. Chem. Res., S. 1996, 314-315.

14. Griffin, G. W.; Manmade, A. J. Org. Chem. 1972, 37, 2589-2600.

15. Marriott, P. J.; Lai, Y. H. J. Chromatogr. A. 1988, 447, 29-41.

16. Willem, R.; Pepermans, H.; Hallenga, K.; Gielen, M.; Dams, R.; Geise, H. J. J. Org. Chem. 1983, 48, 1890-1898.

17. Rigaudy, J.; Scribe, P.; Breviène, C. Tetrahedron 1981, 37, 2585-2593.

18. Durandetti, M.; Perichon, J.; Nedelec, J.-Y. Tetrahedron Lett. 1999, 40, 9009-9013. 


\section{Copies of NMR spectra.}

Compounds 2c (E/Z) and 3c (dl/meso)

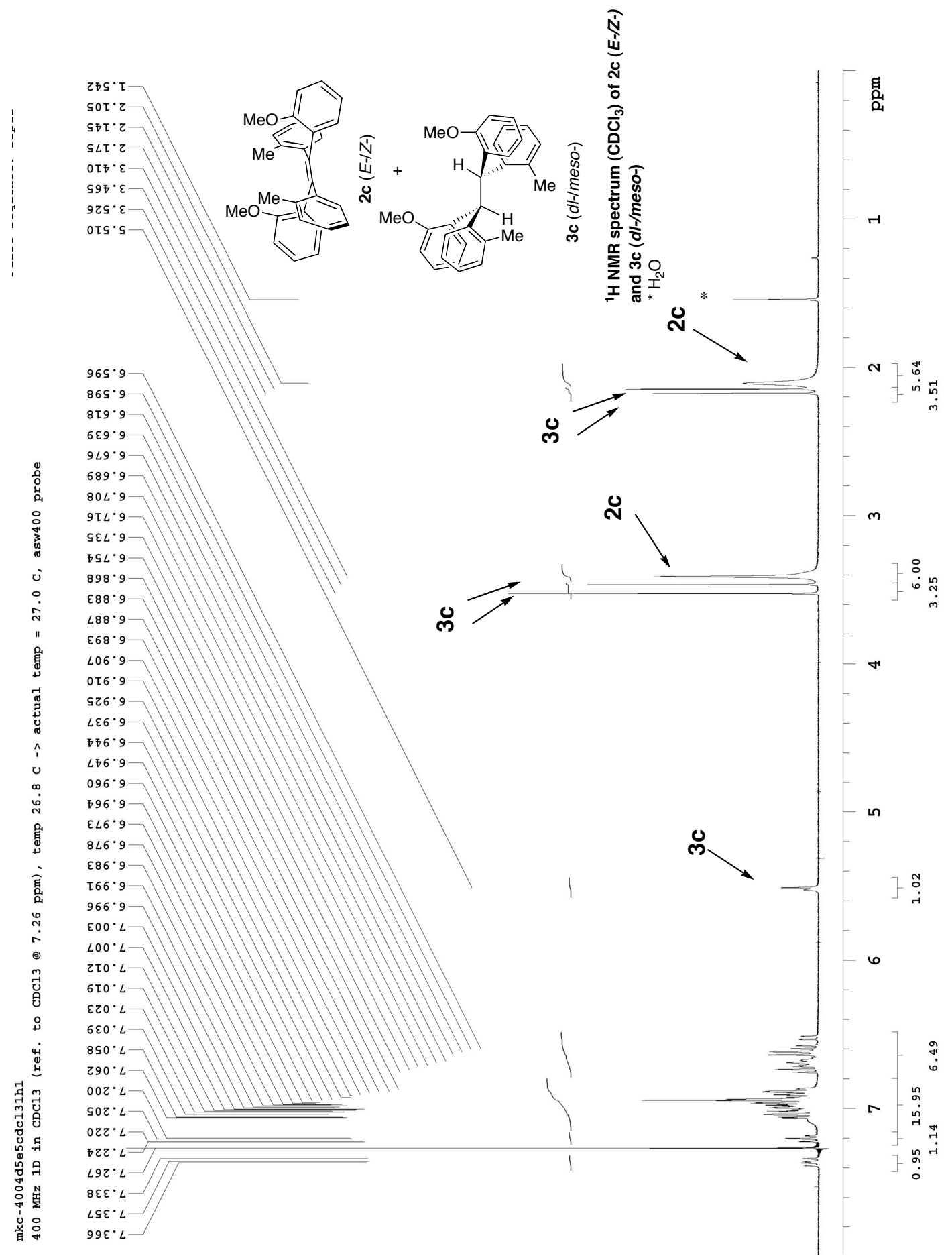




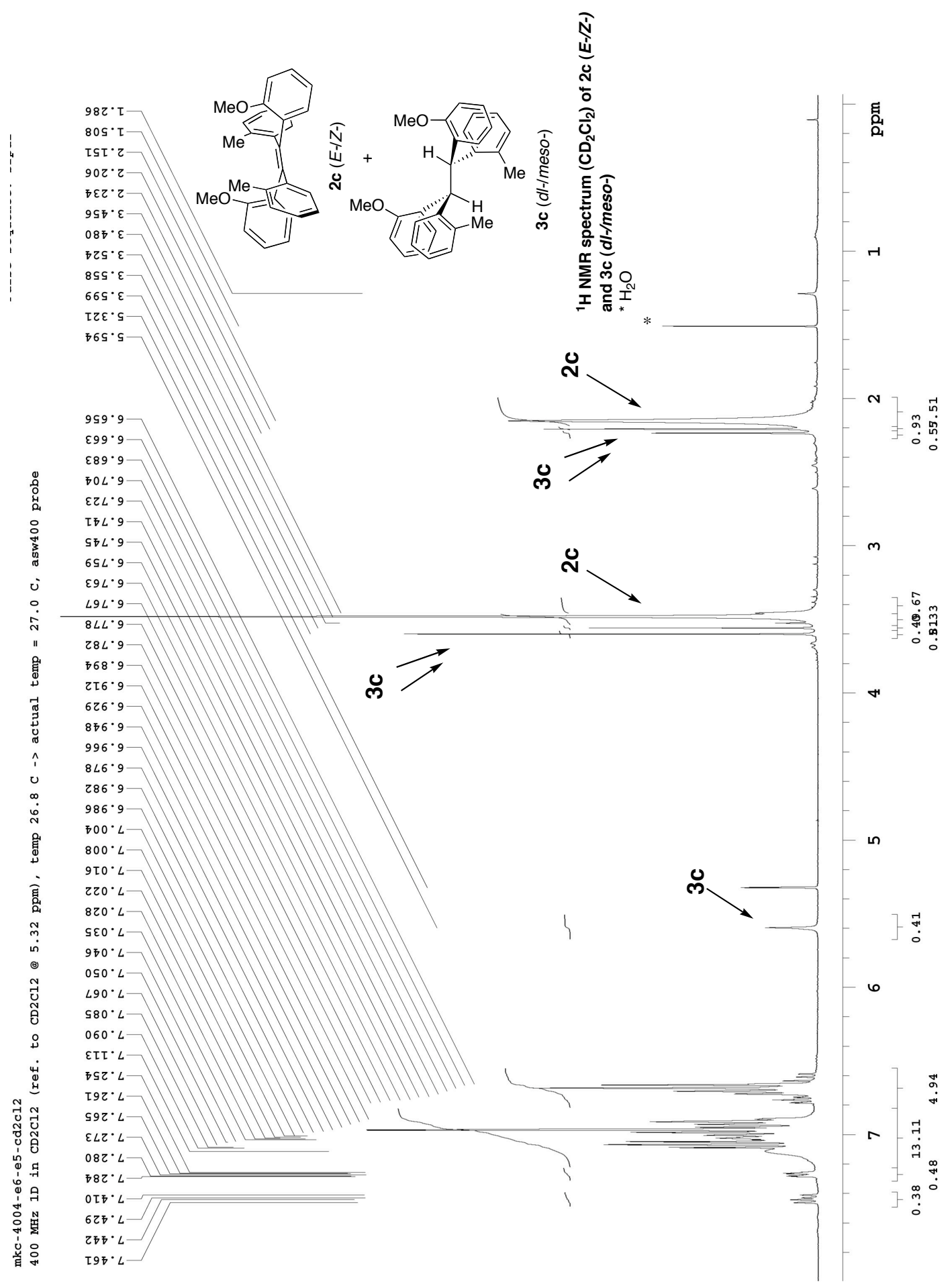




\section{${ }^{13}$ C APT spectrum}

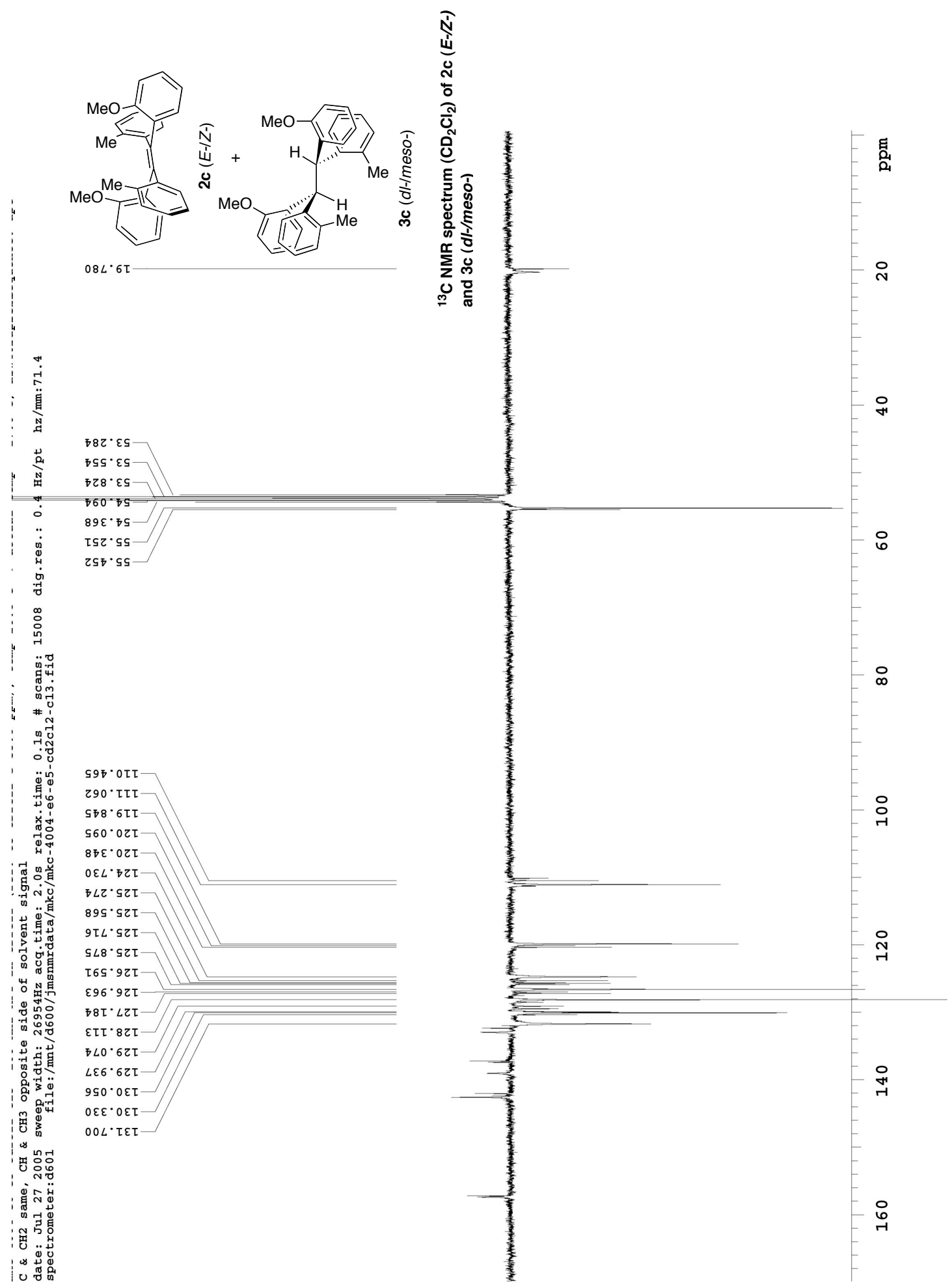




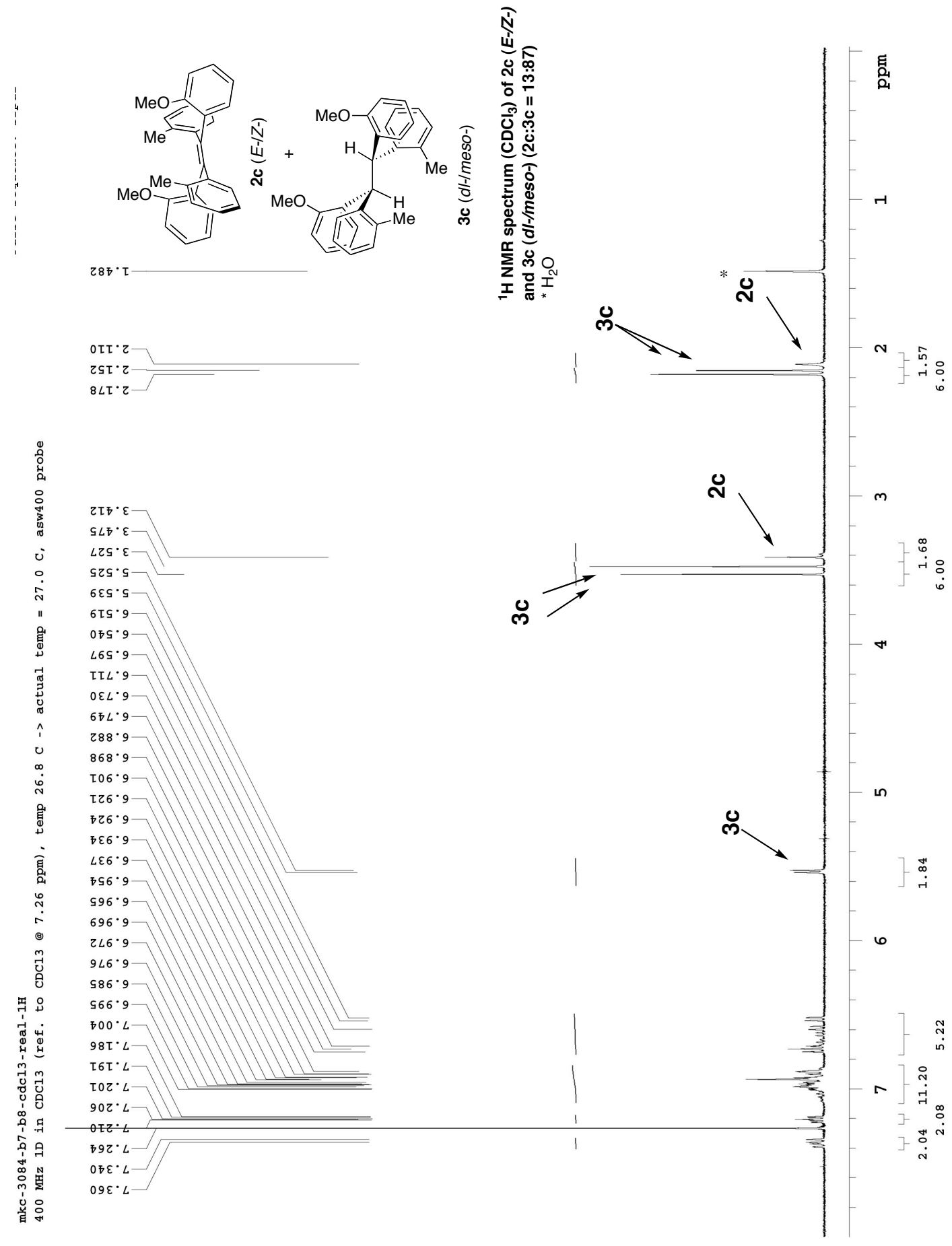




\section{Compound 5}

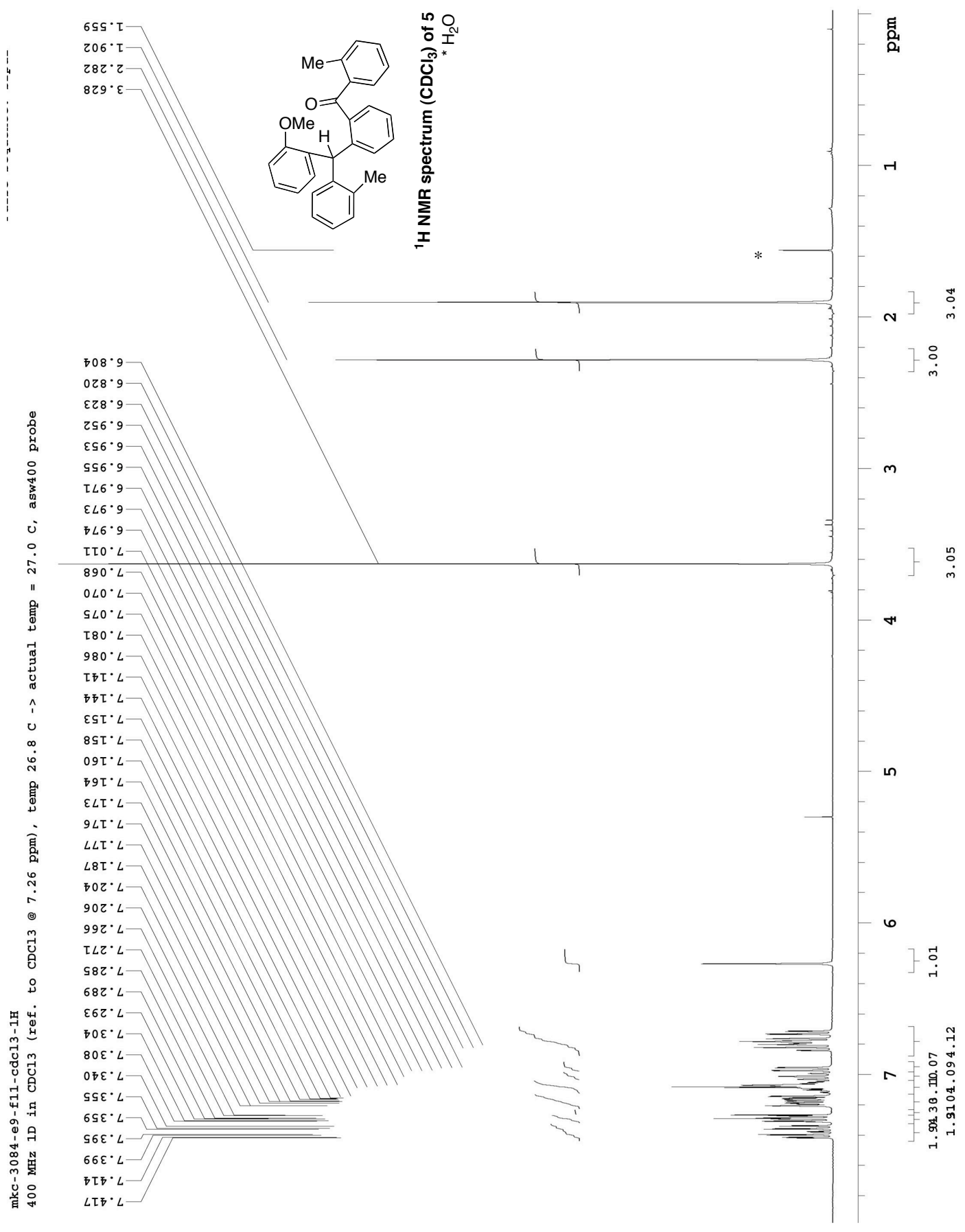



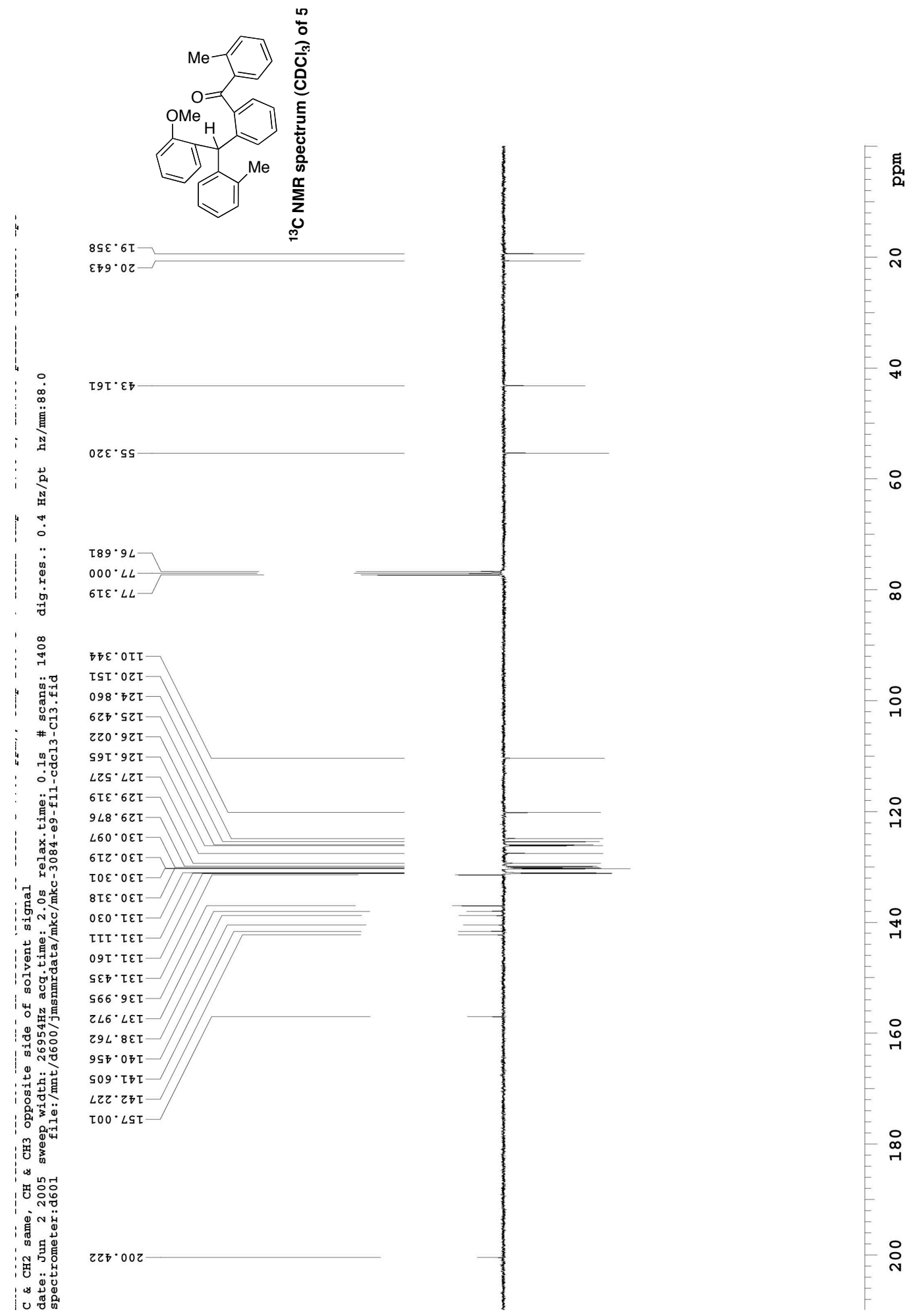


\section{Compound 3e.}

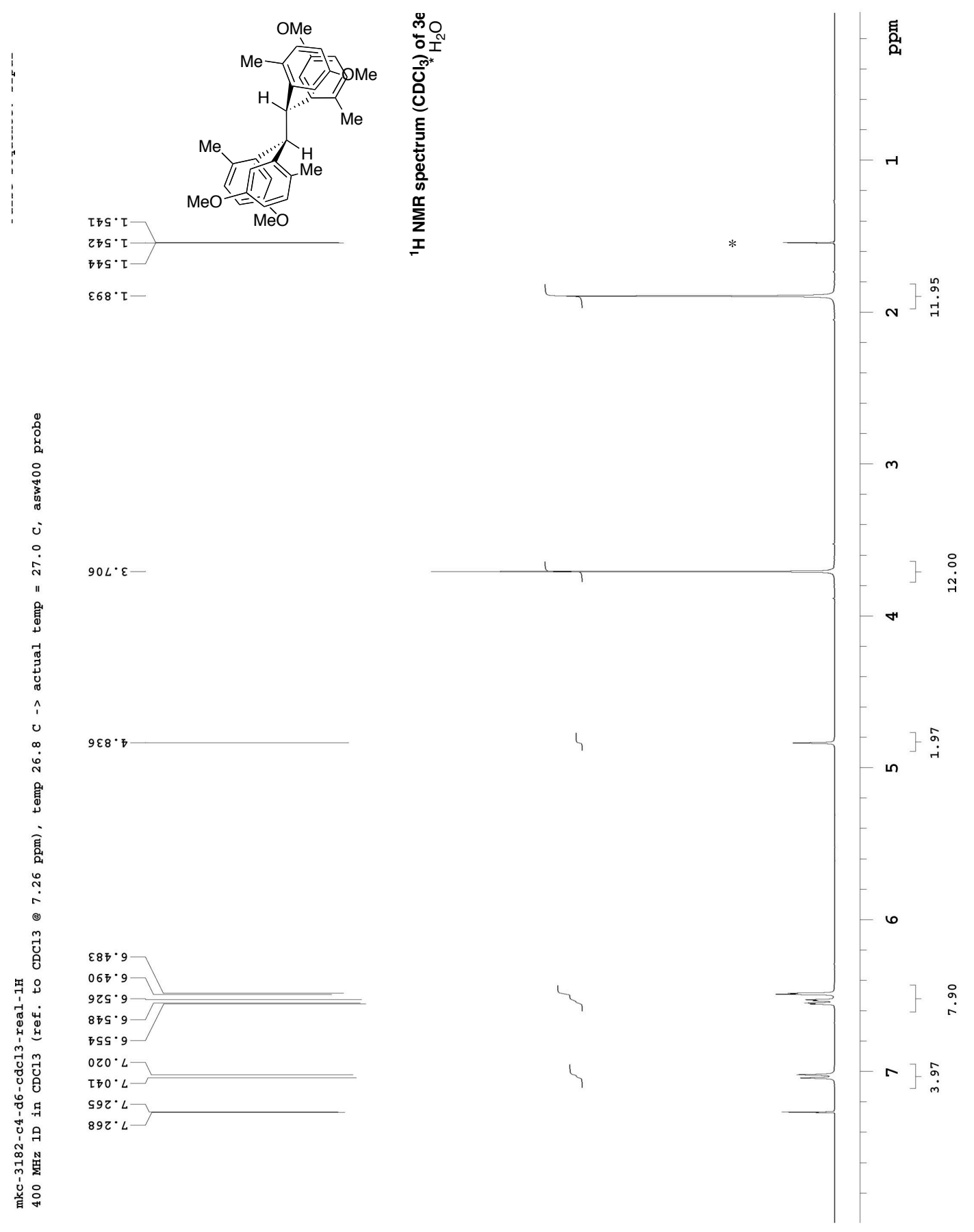




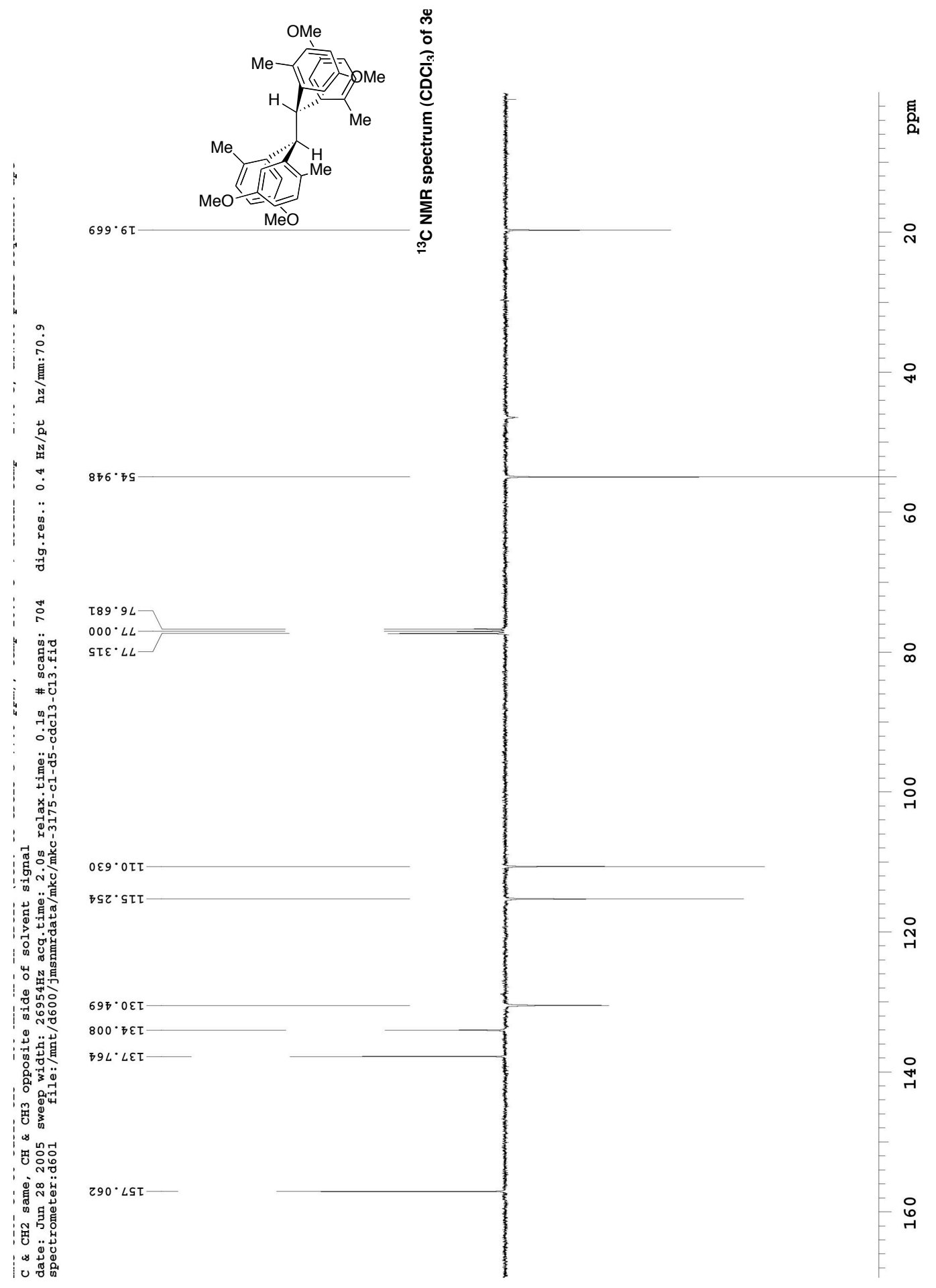




\section{Compound 3f:}

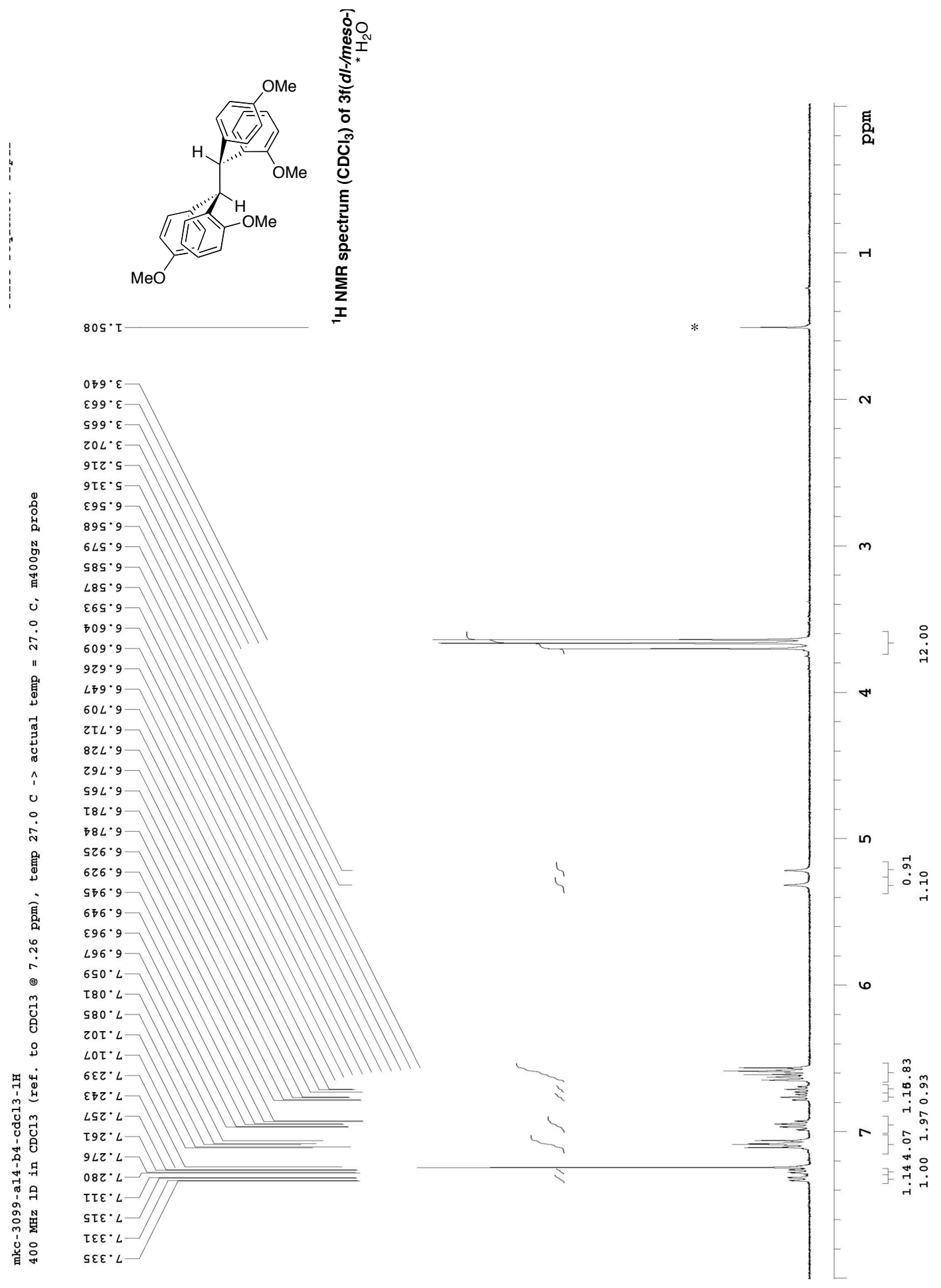




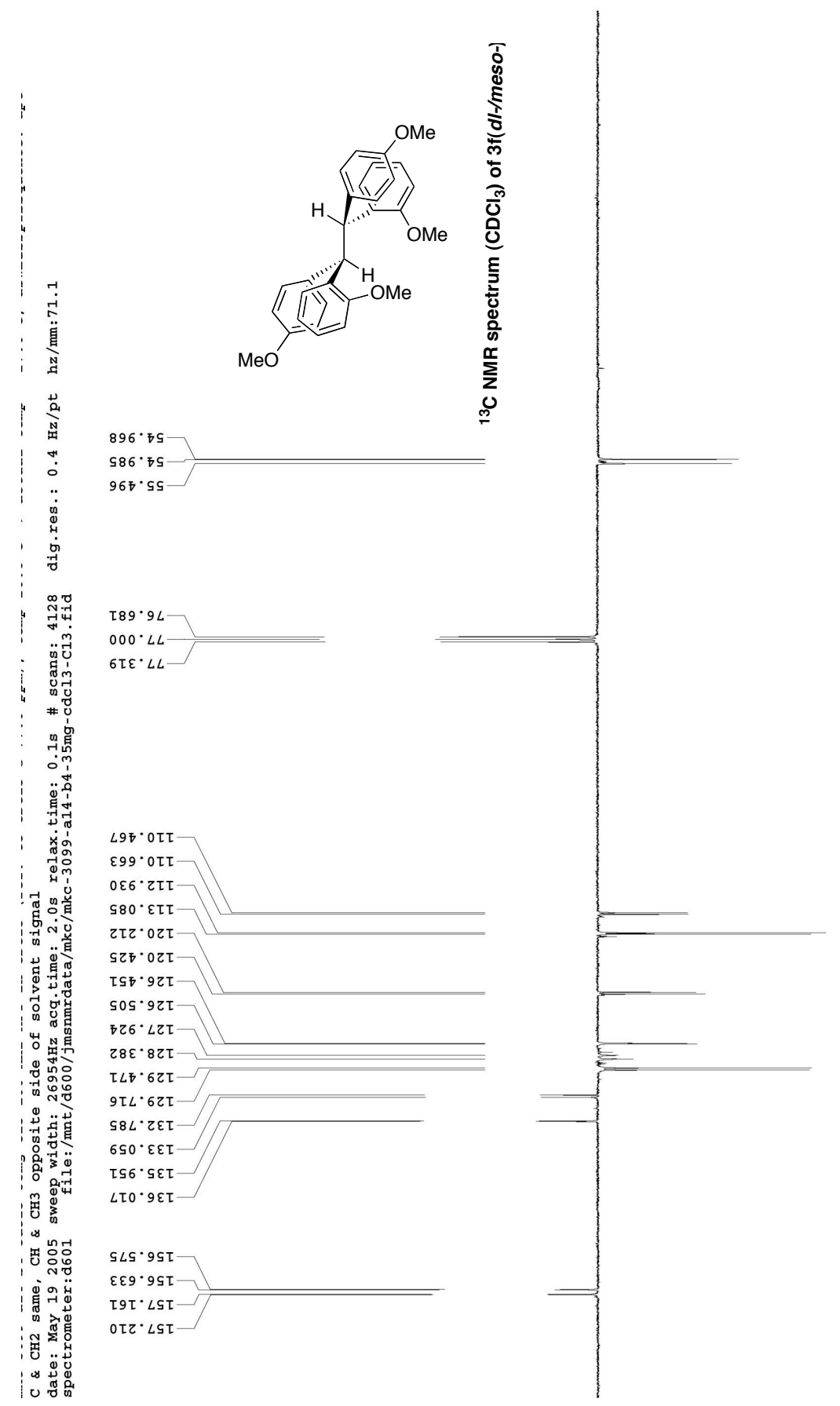

总

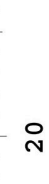

우

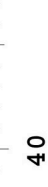

우

:

움

$\stackrel{\text { ㄱ }}{7}$

악

7

$\stackrel{\circ}{\circ}$ 


\section{Compound Z-2g}

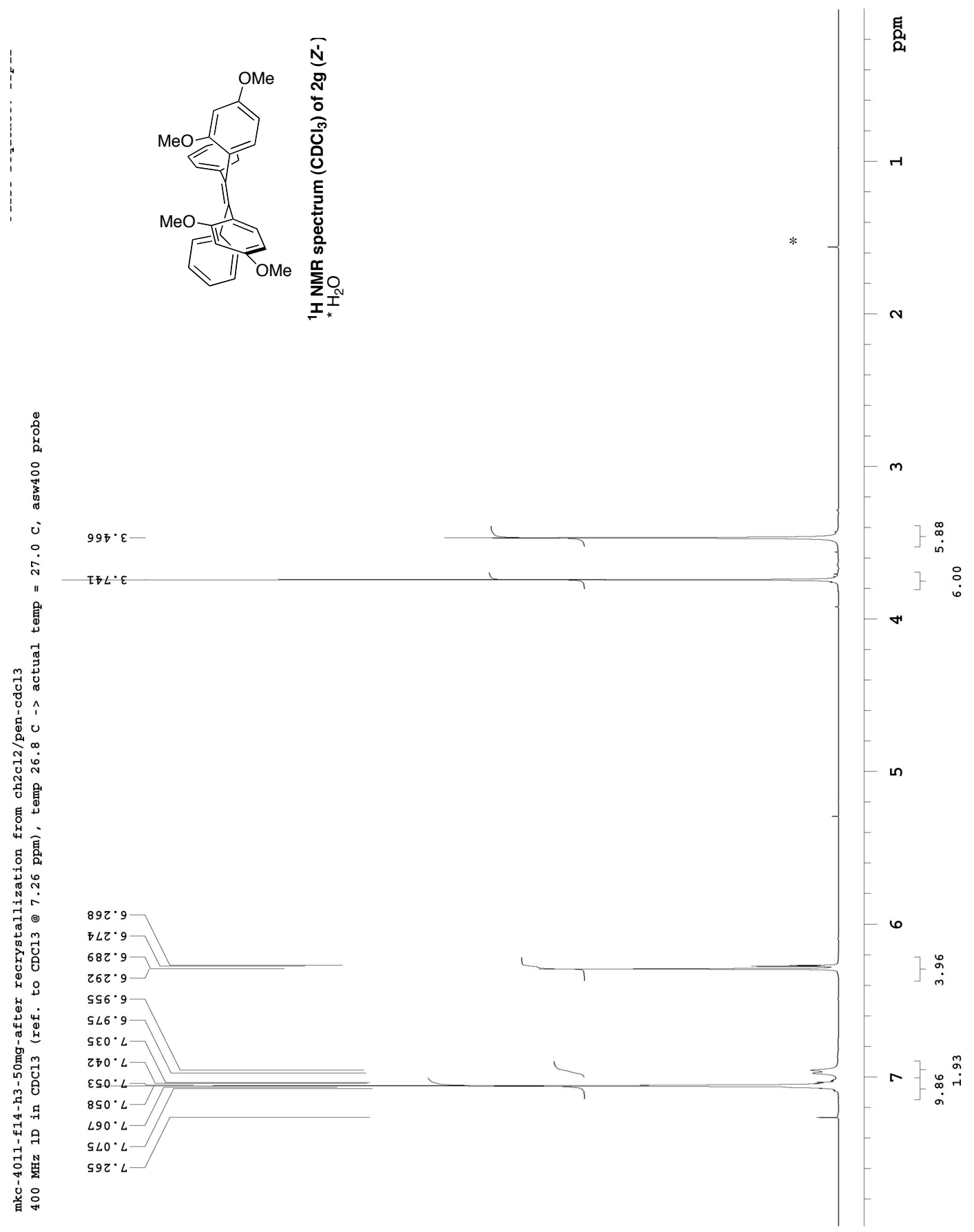




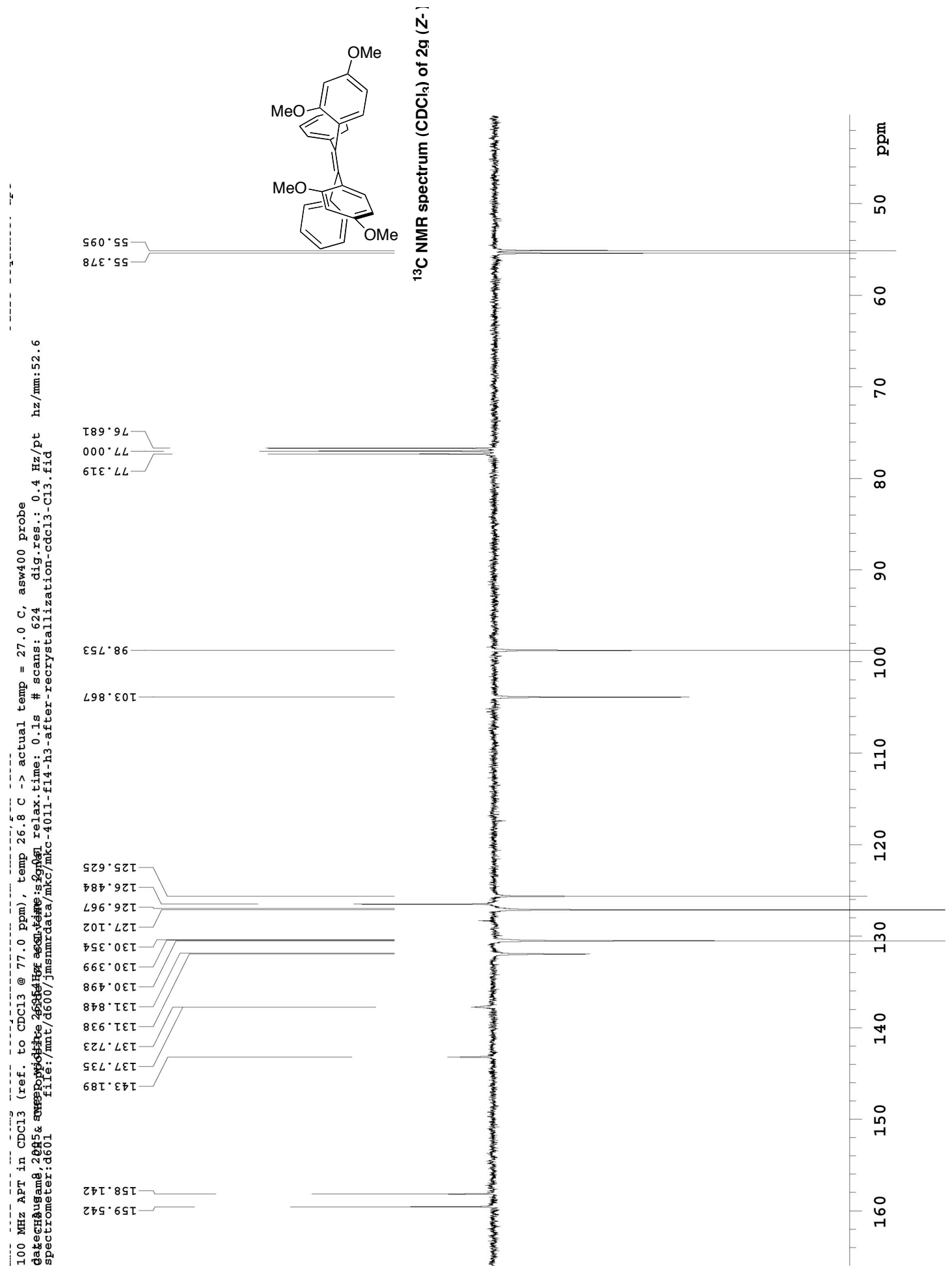




\section{Compound $2 \mathrm{~g}$ ( $E$ and $Z$-isomers)}
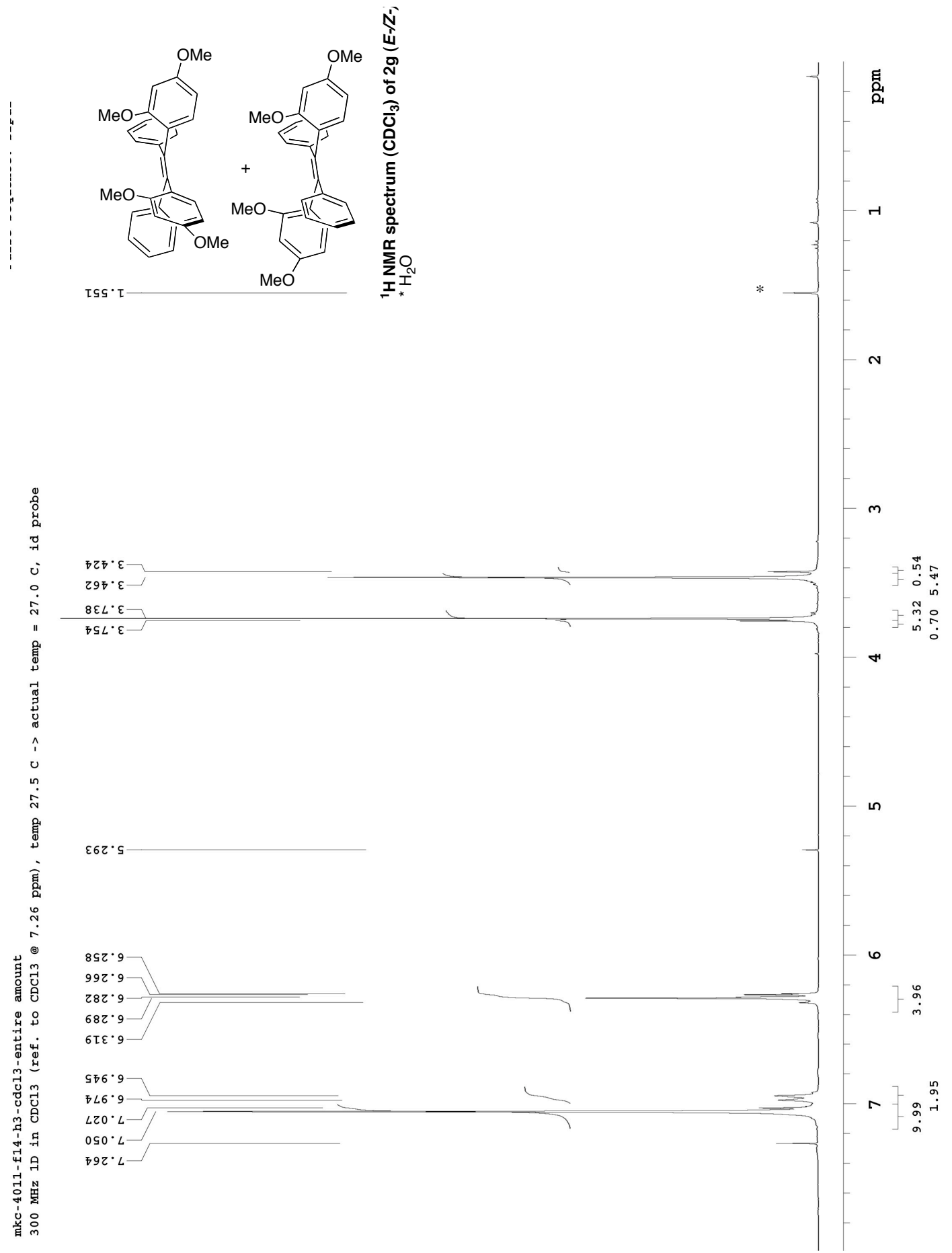


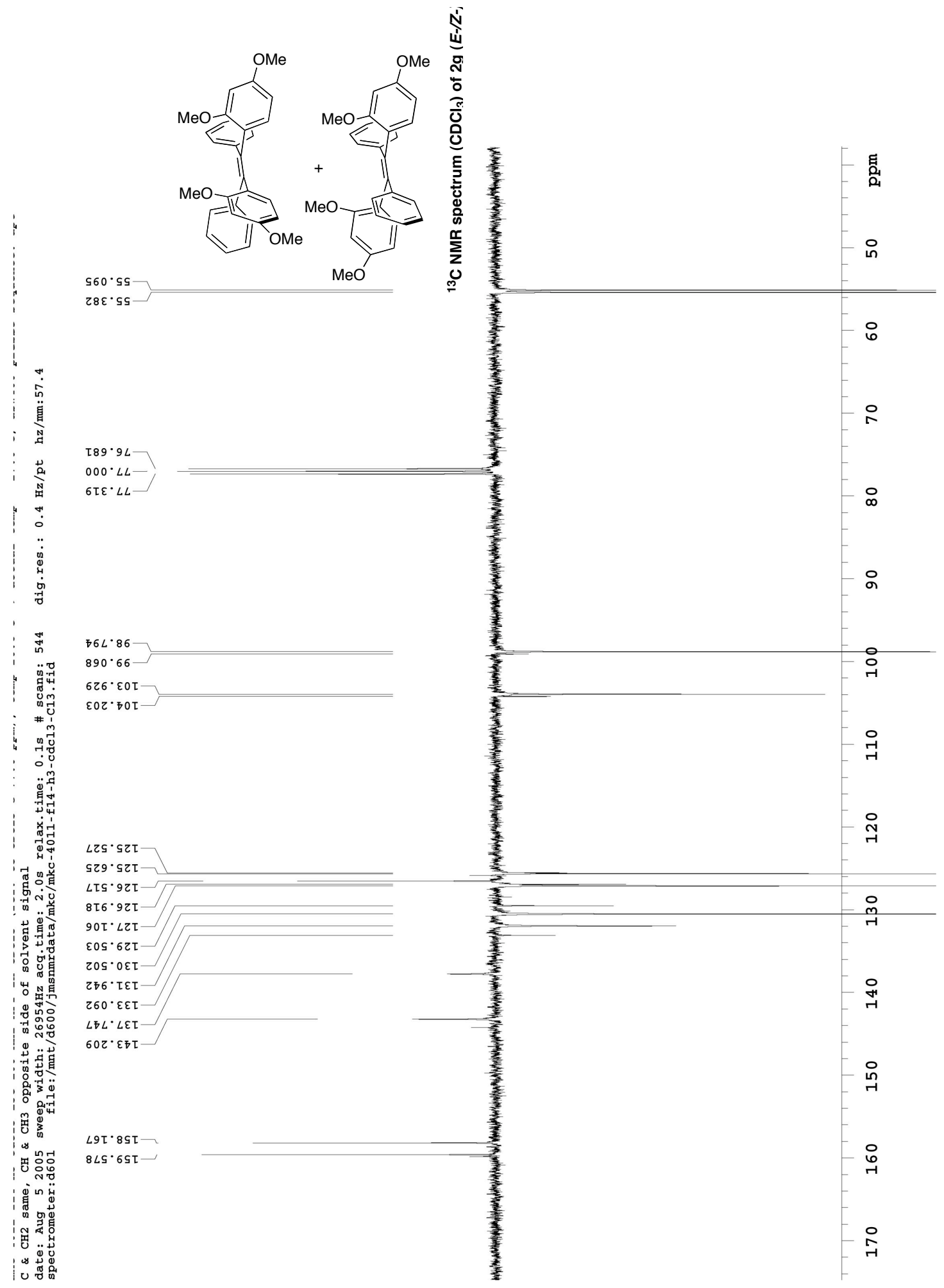




\section{Compound 3g (dl/meso)}

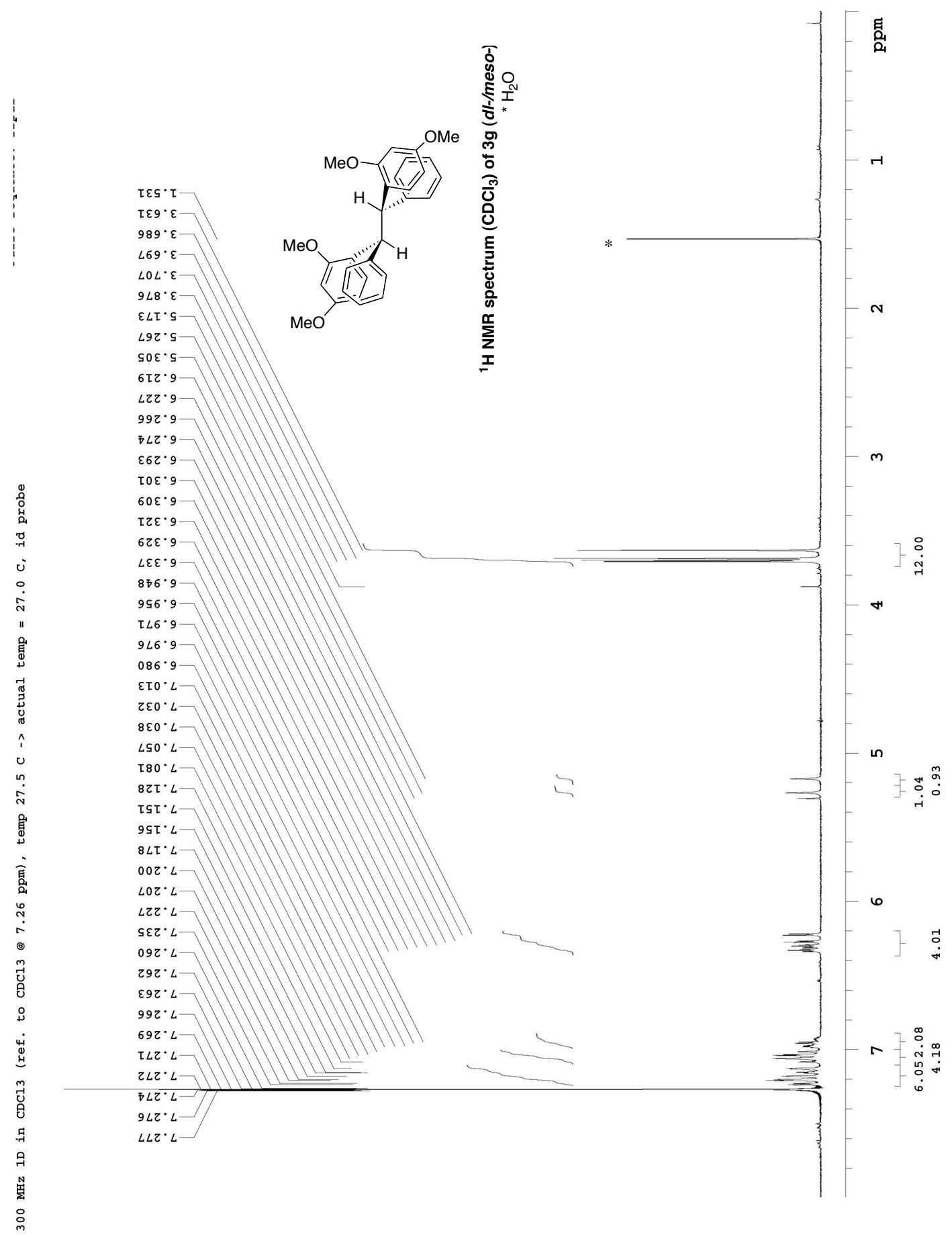




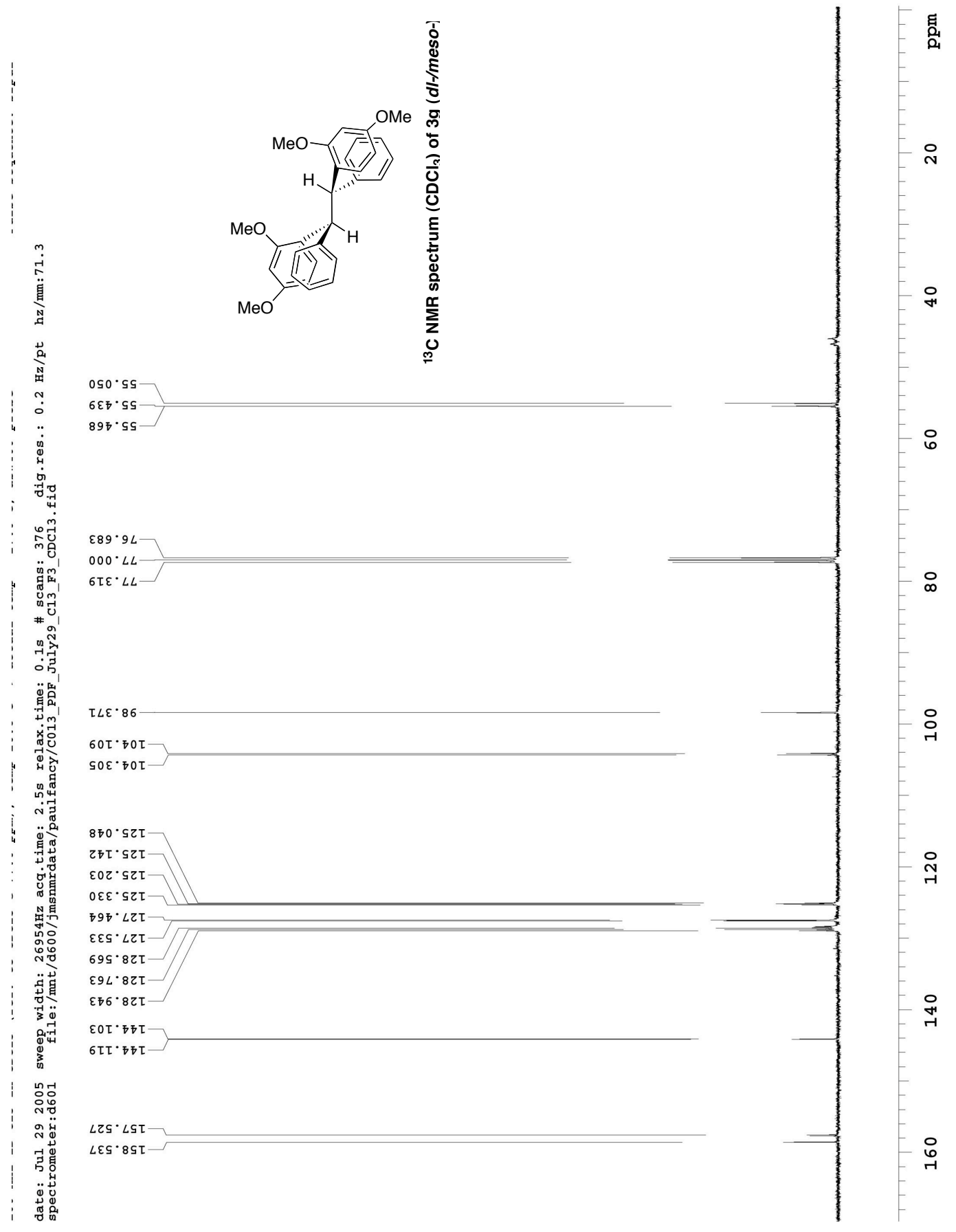




\section{STRUCTURE REPORT}

Compound: (Z)-1,2-Bis(2'-methoxyphenyl)-1,2-bis(phenyl)ethene $\boldsymbol{Z}$-2h. Also named: 1,1'-[(Z)-1,2-diphenylethene-1,2-diyl]bis(2-methoxybenzene).

Formula: $\quad \mathrm{C}_{28} \mathrm{H}_{24} \mathrm{O}_{2}$

Crystallographer:

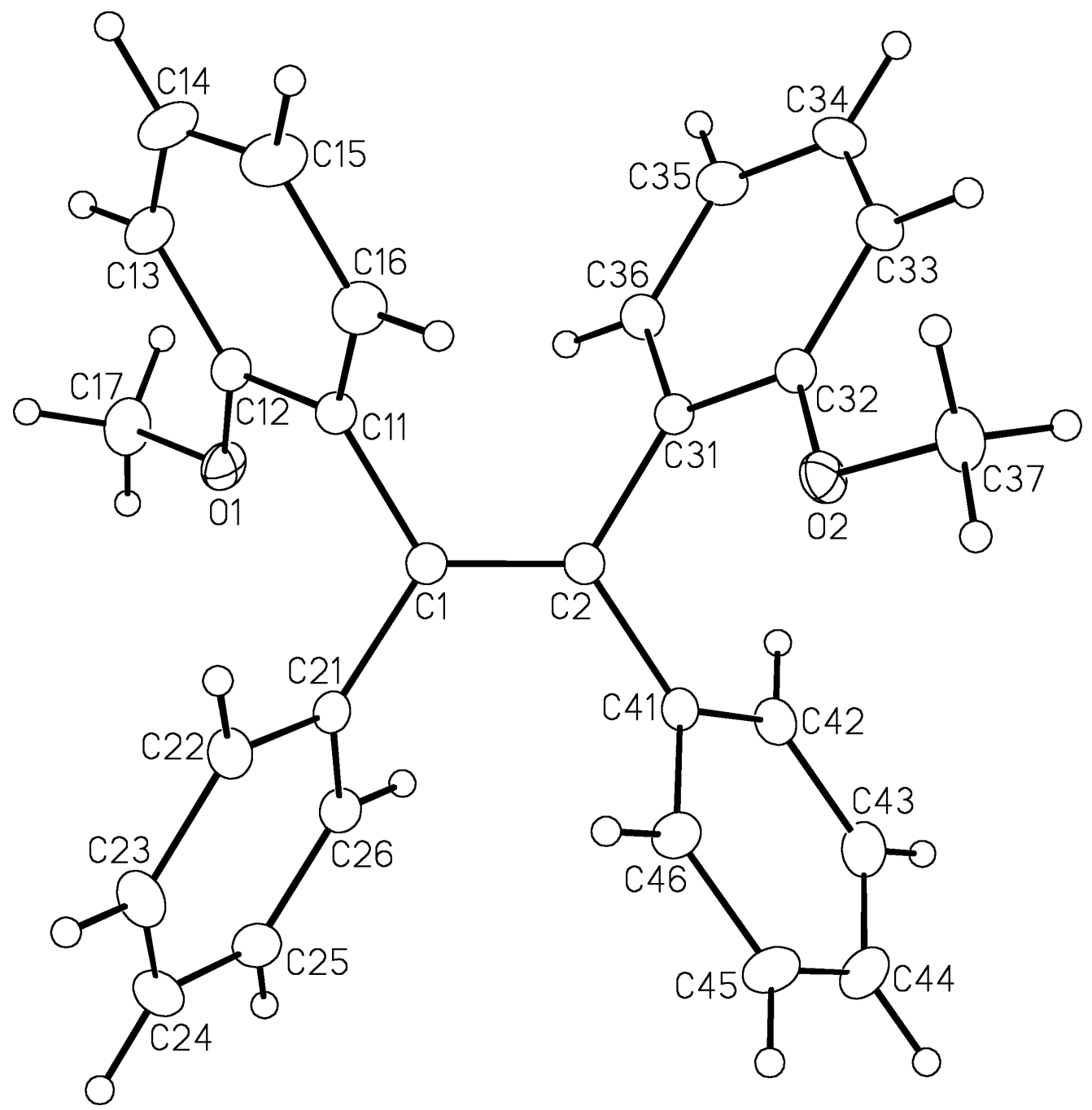

Figure 1. Perspective view of the 1,1'-[(Z)-1,2-diphenylethene-1,2-diyl]bis(2-methoxybenzene) molecule showing the atom labelling scheme. Non-hydrogen atoms are represented by Gaussian ellipsoids at the $20 \%$ probability level. Hydrogen atoms are shown with arbitrarily small thermal parameters. 


\section{List of Tables}

Table 1. Crystallographic Experimental Details

Table 2. Atomic Coordinates and Equivalent Isotropic Displacement Parameters

Table 3. Selected Interatomic Distances

Table 4. Selected Interatomic Angles

Table 5. Anisotropic Displacement Parameters

Table 6. Derived Atomic Coordinates and Displacement Parameters for Hydrogen Atoms

Table 1. Crystallographic Experimental Details

$\begin{array}{ll}\begin{array}{l}\text { A. Crystal Data } \\ \text { formula } \\ \text { formula weight }\end{array} & \mathrm{C}_{28} \mathrm{H}_{24} \mathrm{O}_{2} \\ \text { crystal dimensions }(\mathrm{mm}) & 392.47 \\ \text { crystal system } & 0.55 \times 0.39 \times 0 . \\ \text { space group } & \text { monoclinic } \\ \text { unit cell parameters }{ }^{a} & P 21 / n(\text { an alt } \\ \quad a(\AA) & \\ \quad b(\AA) & 6.0083(4) \\ \quad c(\AA) & 19.6711(14) \\ \quad \beta(\mathrm{deg}) & 18.7067(13) \\ \quad V\left(\AA^{3}\right) & 97.1230(10) \\ \quad Z & 2193.9(3) \\ \rho_{\text {calcd }}\left(\mathrm{g} \mathrm{cm}^{-3}\right) & 4 \\ \mu\left(\mathrm{mm}^{-1}\right) & 1.188\end{array}$

B. Data Collection and Refinement Conditions diffractometer radiation $(\lambda[\AA])$ temperature $\left({ }^{\circ} \mathrm{C}\right)$ Bruker PLATFORM/SMART $1000 \mathrm{CCD}^{b}$ graphite-monochromated Mo K $\alpha(0.71073)$ $-80$ scan type data collection $2 \theta$ limit (deg) $\omega$ scans $\left(0.3^{\circ}\right)(20 \mathrm{~s}$ exposures $)$ 52.80 total data collected $16002(-7 \leq h \leq 7,-24 \leq k \leq 24,-23 \leq l \leq 23)$ independent reflections $4501\left(R_{\text {int }}=0.0250\right)$ 
number of observed reflections $(N O)$

structure solution method

refinement method

(SHELXL-93d)

absorption correction method

range of transmission factors

data/restraints/parameters

goodness-of-fit $(S)^{e}$

final $R$ indices $f$

$$
\begin{aligned}
& R_{1}\left[F_{\mathrm{o}}^{2} \geq 2 \sigma\left(F_{\mathrm{o}}^{2}\right)\right] \\
& w R_{2}\left[F_{\mathrm{o}}^{2} \geq-3 \sigma\left(F_{\mathrm{o}}^{2}\right)\right]
\end{aligned}
$$

largest difference peak and hole
$3568\left[F_{0}^{2} \geq 2 \sigma\left(F_{0}^{2}\right)\right]$

direct methods (SHELXS-86')

full-matrix least-squares on $F^{2}$

multi-scan (SADABS)

0.9905-0.9608

$4501\left[F_{\mathrm{o}}^{2} \geq-3 \sigma\left(F_{\mathrm{o}}^{2}\right)\right] / 0 / 273$

$1.016\left[F_{0}^{2} \geq-3 \sigma\left(F_{0}^{2}\right)\right]$

0.0371

0.1035

0.185 and -0.148 e $\AA^{-3}$

${ }^{a}$ Obtained from least-squares refinement of 7546 reflections with $4.68^{\circ}<2 \theta<52.66^{\circ}$.

$b$ Programs for diffractometer operation, data collection, data reduction and absorption correction were those supplied by Bruker.

cSheldrick, G. M. Acta Crystallogr. 1990, A46, 467-473.

${ }^{d}$ Sheldrick, G. M. SHELXL-93. Program for crystal structure determination. University of Göttingen, Germany, 1993.

${ }^{e} S=\left[\Sigma w\left(F_{\mathrm{o}}^{2}-F_{\mathrm{c}}{ }^{2}\right)^{2} /(n-p)\right]^{1 / 2}(n=$ number of data; $p=$ number of parameters varied; $w$ $=\left[\sigma^{2}\left(F_{\mathrm{o}}^{2}\right)+(0.0462 P)^{2}+0.4809 P\right]^{-1}$ where $\left.P=\left[\operatorname{Max}\left(F_{\mathrm{o}}^{2}, 0\right)+2 F_{\mathrm{c}}^{2}\right] / 3\right)$.

$f_{R_{1}}=\Sigma|| F_{\mathrm{o}}|-| F_{\mathrm{c}}|| / \Sigma\left|F_{\mathrm{o}}\right| ; w R_{2}=\left[\Sigma w\left(F_{\mathrm{o}}^{2}-F_{\mathrm{c}}^{2}\right)^{2} / \Sigma w\left(F_{\mathrm{o}}^{4}\right)\right]^{1 / 2}$. 
Table 2. Atomic Coordinates and Equivalent Isotropic Displacement Parameters

$\begin{array}{lclll}\text { Atom } & x & y & z & U_{\text {eq }} \AA^{2} \\ \text { O1 } & -0.25221(15) & 0.21634(4) & -0.07204(5) & 0.0423(2)^{*} \\ \text { O2 } & 0.32362(14) & 0.21957(4) & 0.22890(4) & 0.0389(2)^{*} \\ \text { C1 } & 0.05598(18) & 0.25629(5) & 0.04193(6) & 0.0284(2)^{*} \\ \text { C2 } & 0.02869(18) & 0.25938(5) & 0.11209(6) & 0.0284(2)^{*} \\ \text { C11 } & 0.07339(19) & 0.18969(6) & 0.00483(6) & 0.0318(3)^{*} \\ \text { C12 } & -0.0831(2) & 0.17101(6) & -0.05354(7) & 0.0360(3)^{*} \\ \text { C13 } & -0.0650(3) & 0.10829(7) & -0.08714(8) & 0.0524(4)^{*} \\ \text { C14 } & 0.1074(3) & 0.06490(8) & -0.06248(10) & 0.0663(5)^{*} \\ \text { C15 } & 0.2617(3) & 0.08211(8) & -0.00500(10) & 0.0640(4)^{*} \\ \text { C16 } & 0.2432(2) & 0.14432(7) & 0.02833(8) & 0.0459(3)^{*} \\ \text { C17 } & -0.4155(2) & 0.19916(8) & -0.13134(8) & 0.0542(4)^{*} \\ \text { C21 } & 0.08309(19) & 0.31854(6) & -0.00199(6) & 0.0297(2)^{*} \\ \text { C22 } & 0.2779(2) & 0.32701(6) & -0.03400(6) & 0.0377(3)^{*} \\ \text { C23 } & 0.3108(2) & 0.38538(7) & -0.07303(7) & 0.0478(3)^{*} \\ \text { C24 } & 0.1486(3) & 0.43520(7) & -0.08111(7) & 0.0490(4)^{*} \\ \text { C25 } & -0.0473(2) & 0.42689(6) & -0.05059(7) & 0.0440(3)^{*} \\ \text { C26 } & -0.0798(2) & 0.36899(6) & -0.01106(6) & 0.0357(3)^{*} \\ \text { C31 } & -0.00801(19) & 0.19652(6) & 0.15372(6) & 0.0300(2)^{*} \\ \text { C32 } & 0.14318(19) & 0.17784(6) & 0.21366(6) & 0.0313(3)^{*} \\ \text { C33 } & 0.1064(2) & 0.11904(6) & 0.25191(7) & 0.0402(3)^{*} \\ \text { C34 } & -0.0803(2) & 0.07947(6) & 0.23082(8) & 0.0455(3)^{*} \\ \text { C35 } & -0.2292(2) & 0.09657(6) & 0.17159(8) & 0.0440(3)^{*} \\ \text { C36 } & -0.1912(2) & 0.15499(6) & 0.13341(7) & 0.0377(3)^{*} \\ \text { C37 } & 0.4821(2) & 0.20340(8) & 0.28982(7) & 0.0469(3)^{*} \\ \text { C41 } & 0.02465(19) & 0.32438(6) & 0.15315(6) & 0.0304(3)^{*} \\ \text { C42 } & -0.1555(2) & 0.33834(6) & 0.19097(7) & 0.0395(3)^{*} \\ \text { C43 } & -0.1611(3) & 0.39791(7) & 0.23052(7) & 0.0511(4)^{*} \\ \text { C44 } & 0.0132(3) & 0.44362(7) & 0.23308(8) & 0.0547(4)^{*} \\ \text { C45 } & 0.1923(3) & 0.43020(7) & 0.19610(8) & 0.0512(4)^{*} \\ \text { C46 } & 0.1991(2) & 0.37108(6) & 0.15630(7) & 0.0393(3)^{*} \\ & & & & \end{array}$

Anisotropically-refined atoms are marked with an asterisk (*). The form of the anisotropic displacement parameter is: $\exp \left[-2 \pi^{2}\left(h^{2} a^{* 2} U_{11}+k^{2} b^{* 2} U_{22}+l^{2} c^{* 2} U_{33}+\right.\right.$ $\left.\left.2 k l b^{*} c^{*} U_{23}+2 h l a^{*} c^{*} U_{13}+2 h k a^{*} b^{*} U_{12}\right)\right]$. 
Table 3. Selected Interatomic Distances $(\AA)$

\begin{tabular}{|c|c|c|c|c|c|}
\hline Atom1 & Atom2 & Distance & Atom1 & Atom2 & Distance \\
\hline $\mathrm{O} 1$ & $\mathrm{C} 12$ & $1.3640(15)$ & $\mathrm{C} 22$ & $\mathrm{C} 23$ & $1.3879(18)$ \\
\hline $\mathrm{O} 1$ & $\mathrm{C} 17$ & $1.4273(14)$ & $\mathrm{C} 23$ & $\mathrm{C} 24$ & $1.377(2)$ \\
\hline $\mathrm{O} 2$ & C32 & $1.3616(14)$ & $\mathrm{C} 24$ & $\mathrm{C} 25$ & $1.380(2)$ \\
\hline $\mathrm{O} 2$ & C37 & $1.4274(14)$ & $\mathrm{C} 25$ & $\mathrm{C} 26$ & $1.3851(17)$ \\
\hline $\mathrm{C} 1$ & $\mathrm{C} 2$ & $1.3440(16)$ & C31 & C32 & $1.4018(15)$ \\
\hline $\mathrm{C} 1$ & C11 & $1.4924(15)$ & C31 & C36 & $1.3854(16)$ \\
\hline $\mathrm{C} 1$ & $\mathrm{C} 21$ & $1.4947(15)$ & C32 & C33 & $1.3921(16)$ \\
\hline $\mathrm{C} 2$ & C31 & $1.4922(15)$ & C33 & C34 & $1.3821(18)$ \\
\hline $\mathrm{C} 2$ & $\mathrm{C} 41$ & $1.4935(15)$ & C34 & $\mathrm{C} 35$ & $1.3770(19)$ \\
\hline C11 & $\mathrm{C} 12$ & $1.3985(16)$ & $\mathrm{C} 35$ & C36 & $1.3869(17)$ \\
\hline C11 & $\mathrm{C} 16$ & $1.3855(17)$ & $\mathrm{C} 41$ & $\mathrm{C} 42$ & $1.3920(17)$ \\
\hline C12 & $\mathrm{C} 13$ & $1.3949(18)$ & $\mathrm{C} 41$ & $\mathrm{C} 46$ & $1.3896(17)$ \\
\hline C13 & $\mathrm{C} 14$ & $1.377(2)$ & $\mathrm{C} 42$ & $\mathrm{C} 43$ & $1.3884(19)$ \\
\hline C14 & $\mathrm{C} 15$ & $1.373(2)$ & $\mathrm{C} 43$ & $\mathrm{C} 44$ & $1.377(2)$ \\
\hline C15 & $\mathrm{C} 16$ & $1.384(2)$ & $\mathrm{C} 44$ & $\mathrm{C} 45$ & $1.375(2)$ \\
\hline $\mathrm{C} 21$ & $\mathrm{C} 22$ & $1.3893(17)$ & $\mathrm{C} 45$ & $\mathrm{C} 46$ & $1.3842(18)$ \\
\hline $\mathrm{C} 21$ & $\mathrm{C} 26$ & $1.3895(16)$ & & & \\
\hline
\end{tabular}


Table 4. Selected Interatomic Angles (deg)

$\begin{array}{llll}\text { Atom1 } & \text { Atom2 } & \text { Atom3 } & \text { Angle } \\ \text { C12 } & \text { O1 } & \text { C17 } & 117.46(10) \\ \text { C32 } & \text { O2 } & \text { C37 } & 117.88(10) \\ \text { C2 } & \text { C1 } & \text { C11 } & 121.19(10) \\ \text { C2 } & \text { C1 } & \text { C21 } & 122.32(10) \\ \text { C11 } & \text { C1 } & \text { C21 } & 116.41(9) \\ \text { C1 } & \text { C2 } & \text { C31 } & 121.06(10) \\ \text { C1 } & \text { C2 } & \text { C41 } & 123.55(10) \\ \text { C31 } & \text { C2 } & \text { C41 } & 115.35(9) \\ \text { C1 } & \text { C11 } & \text { C12 } & 120.97(10) \\ \text { C1 } & \text { C11 } & \text { C16 } & 120.61(11) \\ \text { C12 } & \text { C11 } & \text { C16 } & 118.41(11) \\ \text { O1 } & \text { C12 } & \text { C11 } & 115.72(10) \\ \text { O1 } & \text { C12 } & \text { C13 } & 124.11(12) \\ \text { C11 } & \text { C12 } & \text { C13 } & 120.12(12) \\ \text { C12 } & \text { C13 } & \text { C14 } & 119.65(14) \\ \text { C13 } & \text { C14 } & \text { C15 } & 121.11(13) \\ \text { C14 } & \text { C15 } & \text { C16 } & 119.13(14) \\ \text { C11 } & \text { C16 } & \text { C15 } & 121.58(14) \\ \text { C1 } & \text { C21 } & \text { C22 } & 119.36(10) \\ \text { C1 } & \text { C21 } & \text { C26 } & 121.94(10) \\ \text { C22 } & \text { C21 } & \text { C26 } & 118.69(11) \\ \text { C21 } & \text { C22 } & \text { C23 } & 120.57(12) \\ & & & \\ & & & \end{array}$

Atom1 Atom2 Atom3 Angle

C22 C23 C24 120.08(13)

$\mathrm{C} 23 \quad \mathrm{C} 24 \quad \mathrm{C} 25 \quad 119.91(12)$

$\mathrm{C} 24 \quad \mathrm{C} 25 \quad \mathrm{C} 26 \quad 120.17(12)$

C21 C26 C25 120.57(12)

$\begin{array}{llll}\mathrm{C} 2 & \mathrm{C} 31 & \mathrm{C} 32 & 120.77(10)\end{array}$

$\begin{array}{llll}\mathrm{C} 2 & \mathrm{C} 31 & \mathrm{C} 36 & 120.62(10)\end{array}$

C32 C31 C36 118.61(11)

O2 $\mathrm{C} 32 \quad \mathrm{C} 31 \quad 115.44(10)$

$\begin{array}{llll}\mathrm{O} 2 & \mathrm{C} 32 & \mathrm{C} 33 & 124.50(10)\end{array}$

C31 C32 C33 120.04(11)

$\begin{array}{llll}\mathrm{C} 32 & \mathrm{C} 33 & \mathrm{C} 34 & 119.74(11)\end{array}$

C33 C34 C35 121.05(12)

C34 C35 C36 118.99(12)

C31 C36 C35 121.56(12)

C2 C41 C42 119.58(11)

C2 C41 C46 121.84(11)

$\mathrm{C} 42 \quad \mathrm{C} 41 \quad \mathrm{C} 46 \quad 118.57(11)$

$\mathrm{C} 41 \quad \mathrm{C} 42 \quad \mathrm{C} 43 \quad 120.60(13)$

$\mathrm{C} 42 \mathrm{C} 43 \quad \mathrm{C} 44 \quad 120.07(14)$

$\mathrm{C} 43 \quad \mathrm{C} 44 \quad \mathrm{C} 45 \quad 119.78(13)$

$\mathrm{C} 44 \quad \mathrm{C} 45 \quad \mathrm{C} 46 \quad 120.62(14)$

C41 C46 C45 120.37(13) 
Table 5. Anisotropic Displacement Parameters $\left(U_{\mathrm{ij}}, \AA^{2}\right)$

\begin{tabular}{|c|c|c|c|c|c|c|}
\hline Atom & $U_{11}$ & $U_{22}$ & $U_{33}$ & $U_{23}$ & $U_{13}$ & $U_{12}$ \\
\hline $\mathrm{O} 1$ & $0.0446(5)$ & $0.0455(5)$ & $0.0332(5)$ & $-0.0069(4)$ & $-0.0093(4)$ & 004 \\
\hline 2 & $0.0376(5)$ & $0.0437(5)$ & $0325(5)$ & $0.0064(4)$ & $-0.0072(4)$ & $-0.0024(4)$ \\
\hline 1 & $0.0255(5)$ & $0.0306(6)$ & $0.0277(6)$ & $0.0000(4)$ & $-0.0024(4)$ & 0.0003 \\
\hline 2 & $0.0261(5)$ & $0.0302(6)$ & $273(6)$ & $0.0007(4)$ & $-0.0029(4)$ & $-0.0002(4)$ \\
\hline 11 & $0.0346(6)$ & $0.0311(6)$ & $0.0302(6)$ & $-0.0002(4)$ & $0.0067(5)$ & $0.0000(5)$ \\
\hline 12 & $0.0435(7)$ & $0.0345(6)$ & $0.0308(6)$ & $-0.0034(5)$ & $0.0074(5)$ & $-0.0032(5)$ \\
\hline 13 & $0.0713(10)$ & $0.0412(8)$ & 8) & -0.013 & $0.0085(7)$ & $-0.0081(7)$ \\
\hline 14 & $0.0948(13)$ & 0.038 & $688(11)$ & $-0.0169(7)$ & $0.0225(10)$ & 0.0086 \\
\hline 15 & $0.0712(11)$ & 0.04 & 2) & -0.0028 & $0.0139(9)$ & $0.0236(8)$ \\
\hline 16 & $0.0427(7)$ & 0.04 & 0.04 & $-0.0004(6)$ & $0.0054(6)$ & 0.00 \\
\hline 17 & $0.0505(8)$ & $0.0^{7}$ & 0.03 & $-0.0078(7)$ & $-0.0101(6)$ & $-0.0102(7)$ \\
\hline 21 & 0.034 & 0.0 & 0.02 & $-0.0021(4)$ & $-0.0031(4)$ & $-0.0026(5)$ \\
\hline 22 & $0.0379(6)$ & 0.0 & 0.03 & 0.00 & $0.0023(5)$ & $0.0001(5)$ \\
\hline 23 & $0.0477(8)$ & 0. & .0 & 0.0082 & $0.0042(6)$ & $-0.0125(7)$ \\
\hline 24 & 0.065 & 0.0 & 0 & 0.01 & $-0.0058(7)$ & $-0.0126(7)$ \\
\hline 25 & 0.056 & & 7) & 0.0020 & $-0.0076(6)$ & $0.0037(6)$ \\
\hline 26 & 0.03 & & 0 & -0.001( & $-0.0011(5)$ & $0.0014(5)$ \\
\hline 31 & 0.03 & 0.0 & 6) & -0.000 & $2(5)$ & $0.0011(4)$ \\
\hline 32 & 0.03 & & f & -0 . & 0.0 & 0.00 \\
\hline 33 & 0.04 & & 7) & & 0.0 & $0.0090(5)$ \\
\hline 34 & 0 & & & & 6) & 0.00 \\
\hline 35 & $0 .($ & & & $-0 .($. & 6) & $-0.0087(6)$ \\
\hline 36 & 0.03 & & & 0 & 0.0 & -0.00 \\
\hline 37 & 0.04 & & 7) & ) & -0.0 & $0.0035(6)$ \\
\hline 41 & 0.03 & & 5) & 0078 & $-0.0045(4)$ & $0.0046(5)$ \\
\hline 42 & $0.0445(7)$ & & & & $0.0043(5)$ & $0.0095(5)$ \\
\hline 43 & $0.0656(9)$ & & & & $0.0066(6)$ & $0.0266(7)$ \\
\hline 44 & $0.0789(11)$ & $0.0382(7)$ & & $-0.0104(6)$ & $-0.0127(7)$ & $0.0203(7)$ \\
\hline 4 & $0.0596(9)$ & 0.035 & $0.0532(8)$ & $-0.0055(6)$ & $-0.0157(7)$ & $-0.0008(6)$ \\
\hline C46 & $0.0406(7)$ & $0.0361(6)$ & $0.0389(7)$ & $-0.0031(5)$ & $-0.0048(5)$ & $-0.0002(5)$ \\
\hline
\end{tabular}

The form of the anisotropic displacement parameter is:

$\exp \left[-2 \pi^{2}\left(h^{2} a^{* 2} U_{11}+k^{2} b^{* 2} U_{22}+l^{2} c^{* 2} U_{33}+2 k l b^{*} c^{*} U_{23}+2 h l a^{*} c^{*} U_{13}+2 h k a^{*} b^{*} U_{12}\right)\right]$ 
Table 6. Derived Atomic Coordinates and Displacement Parameters for Hydrogen Atoms

$\begin{array}{lcccc}\text { Atom } & x & y & z & U_{\text {eq }} \AA^{2} \\ \text { H13 } & -0.1711 & 0.0956 & -0.1268 & 0.063 \\ \text { H14 } & 0.1197 & 0.0223 & -0.0857 & 0.080 \\ \text { H15 } & 0.3797 & 0.0517 & 0.0117 & 0.077 \\ \text { H16 } & 0.3495 & 0.1562 & 0.0683 & 0.055 \\ \text { H17A } & -0.5192 & 0.2373 & -0.1419 & 0.065 \\ \text { H17B } & -0.4993 & 0.1589 & -0.1191 & 0.065 \\ \text { H17C } & -0.3401 & 0.1894 & -0.1737 & 0.065 \\ \text { H22 } & 0.3896 & 0.2925 & -0.0291 & 0.045 \\ \text { H23 } & 0.4454 & 0.3910 & -0.0942 & 0.057 \\ \text { H24 } & 0.1714 & 0.4752 & -0.1077 & 0.059 \\ \text { H25 } & -0.1600 & 0.4610 & -0.0567 & 0.053 \\ \text { H26 } & -0.2147 & 0.3637 & 0.0100 & 0.043 \\ \text { H33 } & 0.2093 & 0.1062 & 0.2924 & 0.048 \\ \text { H34 } & -0.1062 & 0.0398 & 0.2576 & 0.055 \\ \text { H35 } & -0.3561 & 0.0688 & 0.1571 & 0.053 \\ \text { H36 } & -0.2932 & 0.1668 & 0.0924 & 0.045 \\ \text { H37A } & 0.5972 & 0.2389 & 0.2965 & 0.056 \\ \text { H37B } & 0.5528 & 0.1596 & 0.2818 & 0.056 \\ \text { H37C } & 0.4051 & 0.2006 & 0.3329 & 0.056 \\ \text { H42 } & -0.2758 & 0.3068 & 0.1897 & 0.047 \\ \text { H43 } & -0.2854 & 0.4071 & 0.2558 & 0.061 \\ \text { H44 } & 0.0098 & 0.4843 & 0.2603 & 0.066 \\ \text { H45 } & 0.3124 & 0.4618 & 0.1979 & 0.061 \\ \text { H46 } & 0.3238 & 0.3624 & 0.1310 & 0.047\end{array}$




\section{STRUCTURE REPORT}

Compound: 1,1,2,2-Tetrakis(4-methoxy-2-methylphenyl)ethane, hemi(dichloromethane) solvate 3e

Formula: $\quad \mathrm{C}_{34.5} \mathrm{H}_{39} \mathrm{ClO}_{4}\left(\mathrm{C}_{34} \mathrm{H}_{38} \mathrm{O}_{4} \cdot 0.5 \mathrm{CH}_{2} \mathrm{Cl}_{2}\right)$

Crystallographer: R. McDonald

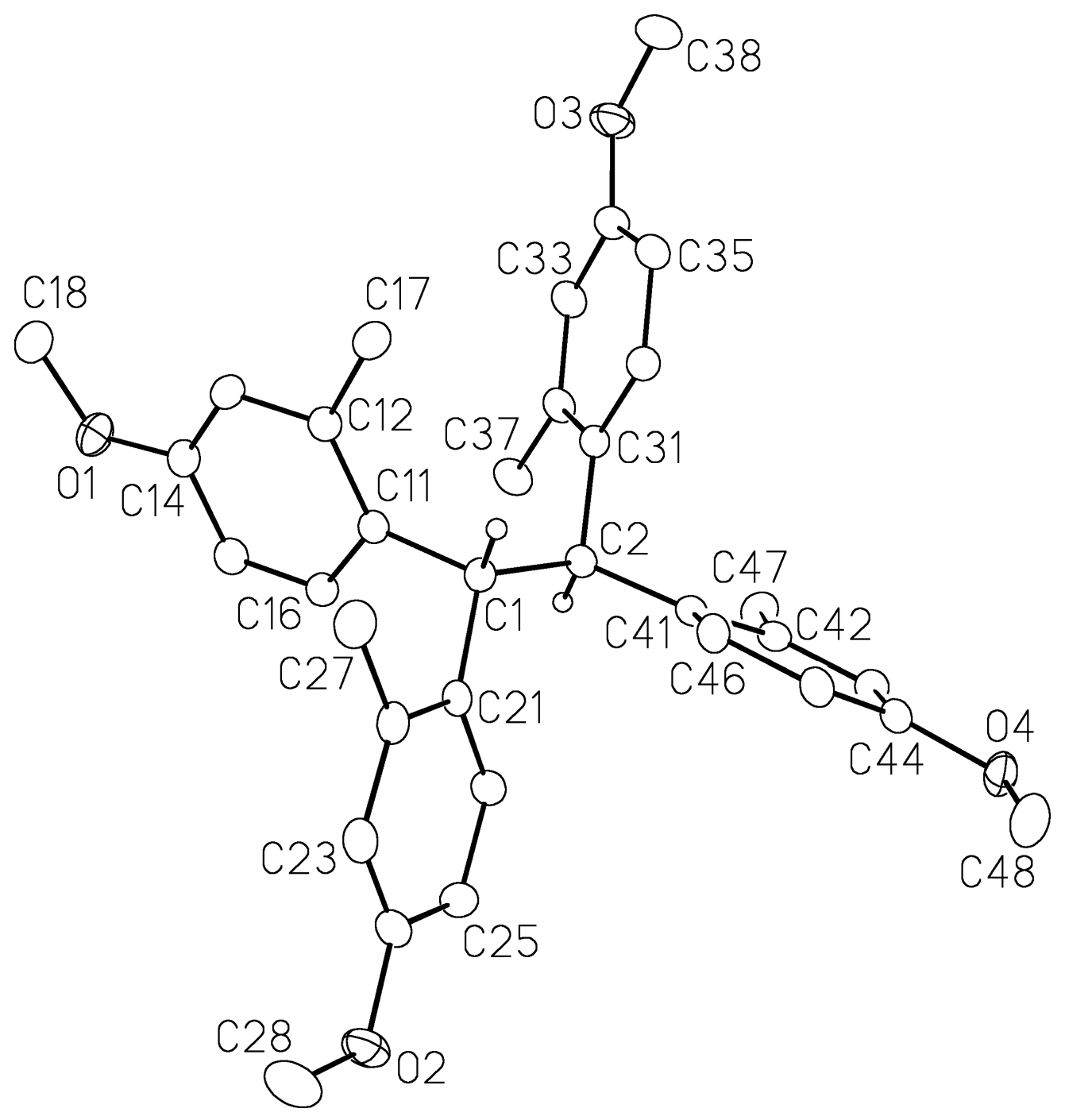

Figure 1. Perspective view of the 1,1,2,2-tetrakis(4-methoxy-2-methylphenyl)ethane molecule showing the atom labelling scheme. Non-hydrogen atoms are represented by Gaussian ellipsoids at the $20 \%$ probability level. The hydrogen atoms attached to $\mathrm{C} 1$ and $\mathrm{C} 2$ are shown with arbitrarily small thermal parameters; all other hydrogens are not shown. 


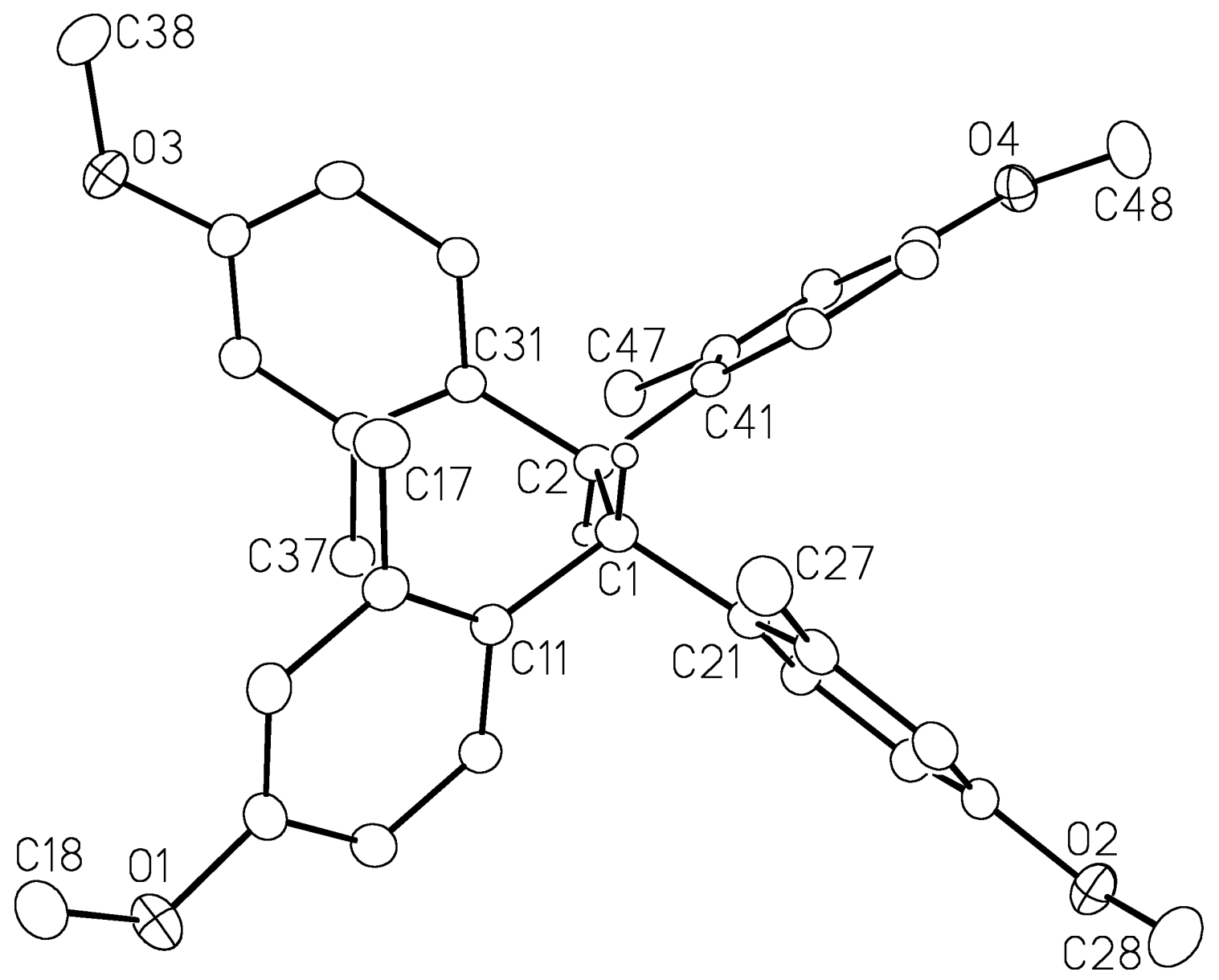

Figure 2. Alternate view of the molecule slightly offset from along the $\mathrm{C} 1-\mathrm{C} 2$ bond. 


\section{List of Tables}

Table 1. Crystallographic Experimental Details

Table 2. Atomic Coordinates and Equivalent Isotropic Displacement Parameters

Table 3. Selected Interatomic Distances

Table 4. Selected Interatomic Angles

Table 5. Anisotropic Displacement Parameters

Table 6. Derived Atomic Coordinates and Displacement Parameters for Hydrogen Atoms

Table 1. Crystallographic Experimental Details

$\begin{array}{ll}\begin{array}{l}\text { A. Crystal Data } \\ \text { formula }\end{array} & \\ \text { formula weight } & \mathrm{C}_{34.5} \mathrm{H}_{39} \mathrm{ClO}_{4} \\ \text { crystal dimensions }(\mathrm{mm}) & 553.11 \\ \text { crystal system } & 0.45 \times 0.37 \times 0.13 \\ \text { space group } & \text { monoclinic } \\ \text { unit cell parameters } a & C 2 / c(\text { No. } 15) \\ \quad a(\AA) & \\ \quad b(\AA) & 25.1768(18) \\ \quad c(\AA) & 11.7431(8) \\ \quad \beta(\mathrm{deg}) & 20.5459(15) \\ \quad V\left(\AA^{3}\right) & 105.8900(12) \\ \quad Z & 5842.4(7) \\ \rho_{\text {calcd }}\left(\mathrm{g} \mathrm{cm}^{-3}\right) & 8 \\ \mu\left(\mathrm{mm}^{-1}\right) & 1.258\end{array}$

B. Data Collection and Refinement Conditions diffractometer radiation $(\lambda[\AA])$

temperature $\left({ }^{\circ} \mathrm{C}\right)$

scan type

data collection $2 \theta$ limit (deg)

total data collected 25)
Bruker PLATFORM/SMART $1000 \mathrm{CCD}^{b}$ graphite-monochromated Mo K $\alpha(0.71073)$ $-80$ $\omega$ scans $\left(0.3^{\circ}\right)(20 \mathrm{~s}$ exposures $)$ 52.88 $22831(-31 \leq h \leq 31,-14 \leq k \leq 14,-25 \leq l \leq$ 
independent reflections

number of observed reflections $(\mathrm{NO})$

structure solution method

refinement method

(SHELXL-93d)

absorption correction method

range of transmission factors

data/restraints/parameters

goodness-of-fit $(S)^{e}$

final $R$ indices $f$

$$
R_{1}\left[F_{\mathrm{o}}^{2} \geq 2 \sigma\left(F_{\mathrm{o}}^{2}\right)\right]
$$$$
w R_{2}\left[F_{\mathrm{o}}^{2} \geq-3 \sigma\left(F_{\mathrm{o}}^{2}\right)\right]
$$

largest difference peak and hole
$6017\left(R_{\text {int }}=0.0419\right)$

$4071\left[F_{\mathrm{o}}^{2} \geq 2 \sigma\left(F_{\mathrm{o}}^{2}\right)\right]$

direct methods (SHELXS-86c)

full-matrix least-squares on $F^{2}$

multi-scan (SADABS)

0.9785-0.9281

$6017\left[F_{\mathrm{o}}^{2} \geq-3 \sigma\left(F_{\mathrm{o}}^{2}\right)\right] / 0 / 361$

$1.031\left[F_{\mathrm{o}}^{2} \geq-3 \sigma\left(F_{\mathrm{o}}{ }^{2}\right)\right]$

${ }^{a}$ Obtained from least-squares refinement of 7553 reflections with $4.58^{\circ}<2 \theta<51.80^{\circ}$.

$b$ Programs for diffractometer operation, data collection, data reduction and absorption correction were those supplied by Bruker.

cSheldrick, G. M. Acta Crystallogr. 1990, A46, 467-473.

dSheldrick, G. M. SHELXL-93. Program for crystal structure determination. University of Göttingen, Germany, 1993.

$e S=\left[\sum w\left(F_{\mathrm{o}}^{2}-F_{\mathrm{c}^{2}}\right)^{2} /(n-p)\right]^{1 / 2}(n=$ number of data; $p=$ number of parameters varied; $w$ $=\left[\sigma^{2}\left(F_{\mathrm{o}}^{2}\right)+(0.1247 P)^{2}+6.9200 P\right]^{-1}$ where $\left.P=\left[\operatorname{Max}\left(F_{\mathrm{o}}^{2}, 0\right)+2 F_{\mathrm{c}}^{2}\right] / 3\right)$.

$f_{R_{1}}=\Sigma|| F_{\mathrm{o}}|-| F_{\mathrm{c}}|| / \Sigma\left|F_{\mathrm{o}}\right| ; w R_{2}=\left[\Sigma w\left(F_{\mathrm{o}}^{2}-F_{\mathrm{c}}{ }^{2}\right)^{2} / \Sigma w\left(F_{\mathrm{o}}{ }^{4}\right)\right]^{1 / 2}$. 
Table 2. Atomic Coordinates and Equivalent Isotropic Displacement Parameters

(a) atoms of 1,1,2,2-tetrakis(4-methoxy-2-methylphenyl)ethane

\begin{tabular}{|c|c|c|c|c|}
\hline Atom & $x$ & $y$ & $z$ & $U_{\text {eq }}, \AA^{2}$ \\
\hline $\mathrm{O} 1$ & $0.07058(10)$ & $-0.0883(2)$ & $0.32136(12)$ & $0.0605(6)^{*}$ \\
\hline $\mathrm{O} 2$ & $0.40112(9)$ & $0.1672(2)$ & $0.32175(13)$ & $0.0631(6)^{*}$ \\
\hline $\mathrm{O} 3$ & $-0.07937(9)$ & $0.3171(2)$ & $0.04275(14)$ & $0.0705(7)^{*}$ \\
\hline $\mathrm{O} 4$ & $0.27680(9)$ & $0.54800(19)$ & $-0.00202(10)$ & $0.0534(6)^{*}$ \\
\hline $\mathrm{C} 1$ & $0.17315(10)$ & $0.2570(2)$ & $0.20251(12)$ & $0.0345(6)^{*}$ \\
\hline $\mathrm{C} 2$ & $0.15377(10)$ & $0.2542(2)$ & $0.12396(12)$ & $0.0340(6)^{*}$ \\
\hline $\mathrm{C} 11$ & $0.14210(10)$ & $0.1700(2)$ & $0.23417(12)$ & $0.0347(6)^{*}$ \\
\hline $\mathrm{C} 12$ & $0.10020(11)$ & $0.1993(2)$ & $0.26361(13)$ & $0.0402(6)^{*}$ \\
\hline C13 & $0.07563(12)$ & $0.1130(3)$ & $0.29332(14)$ & $0.0443(7)^{*}$ \\
\hline $\mathrm{C} 14$ & $0.09197(12)$ & $0.0012(2)$ & $0.29343(14)$ & $0.0425(6)^{*}$ \\
\hline $\mathrm{C} 15$ & $0.13374(12)$ & $-0.0277(2)$ & $0.26459(14)$ & $0.0432(7)^{*}$ \\
\hline $\mathrm{C} 16$ & $0.15794(11)$ & $0.0559(2)$ & $0.23598(13)$ & $0.0380(6)^{*}$ \\
\hline $\mathrm{C} 17$ & $0.07909(14)$ & $0.3190(3)$ & $0.26620(17)$ & $0.0543(8)^{*}$ \\
\hline $\mathrm{C} 18$ & $0.03350(15)$ & $-0.0605(3)$ & $0.35977(19)$ & $0.0652(9)^{*}$ \\
\hline $\mathrm{C} 21$ & $0.23500(11)$ & $0.2397(2)$ & $0.23270(13)$ & $0.0357(6)^{*}$ \\
\hline $\mathrm{C} 22$ & $0.26036(12)$ & $0.2809(2)$ & $0.29794(13)$ & $0.0415(6)^{*}$ \\
\hline $\mathrm{C} 23$ & $0.31548(12)$ & $0.2567(3)$ & $0.32829(15)$ & $0.0467(7)^{*}$ \\
\hline $\mathrm{C} 24$ & $0.34613(11)$ & $0.1947(2)$ & $0.29550(16)$ & $0.0472(7)^{*}$ \\
\hline $\mathrm{C} 25$ & $0.32195(12)$ & $0.1545(2)$ & $0.22959(16)$ & $0.0469(7)^{*}$ \\
\hline $\mathrm{C} 26$ & $0.26684(11)$ & $0.1783(2)$ & $0.19955(14)$ & $0.0407(6)^{*}$ \\
\hline $\mathrm{C} 27$ & $0.22915(14)$ & $0.3515(3)$ & $0.33615(16)$ & $0.0544(8)^{*}$ \\
\hline $\mathrm{C} 28$ & $0.42599(16)$ & $0.2075(4)$ & $0.3888(2)$ & $0.0791(12)^{*}$ \\
\hline C31 & $0.09142(11)$ & $0.2711(2)$ & $0.09880(12)$ & $0.0351(6)^{*}$ \\
\hline $\mathrm{C} 32$ & $0.05434(11)$ & $0.1803(2)$ & $0.07605(14)$ & $0.0416(6)^{*}$ \\
\hline $\mathrm{C} 33$ & $-0.00190(12)$ & $0.2024(3)$ & $0.05759(16)$ & $0.0501(7)^{*}$ \\
\hline $\mathrm{C} 34$ & $-0.02277(12)$ & $0.3097(3)$ & $0.06049(16)$ & $0.0506(7)^{*}$ \\
\hline $\mathrm{C} 35$ & $0.01284(12)$ & $0.4003(3)$ & $0.08039(16)$ & $0.0484(7)^{*}$ \\
\hline $\mathrm{C} 36$ & $0.06934(11)$ & $0.3790(2)$ & $0.09915(14)$ & $0.0407(6)^{*}$ \\
\hline $\mathrm{C} 37$ & $0.07297(13)$ & $0.0595(2)$ & $0.07108(16)$ & $0.0508(7)^{*}$ \\
\hline $\mathrm{C} 38$ & $-0.10397(15)$ & $0.4261(3)$ & $0.0438(2)$ & $0.0745(11)^{*}$ \\
\hline $\mathrm{C} 41$ & $0.18492(10)$ & $0.3386(2)$ & $0.09039(12)$ & $0.0338(6)^{*}$ \\
\hline $\mathrm{C} 42$ & $0.18556(10)$ & $0.3224(2)$ & $0.02263(13)$ & $0.0360(6)^{*}$ \\
\hline $\mathrm{C} 43$ & $0.21618(11)$ & $0.3955(2)$ & $-0.00522(13)$ & $0.0413(6)^{*}$ \\
\hline $\mathrm{C} 44$ & $0.24621(11)$ & $0.4851(2)$ & $0.03124(14)$ & $0.0406(6) *$ \\
\hline $\mathrm{C} 45$ & $0.24381(12)$ & $0.5051(2)$ & $0.09646(13)$ & $0.0408(6)^{*}$ \\
\hline $\mathrm{C} 46$ & $0.21312(11)$ & $0.4315(2)$ & $0.12459(13)$ & $0.0396(6)^{*}$ \\
\hline $\mathrm{C} 47$ & $0.15360(12)$ & $0.2279(3)$ & $-0.02001(14)$ & $0.0481(7)^{*}$ \\
\hline $\mathrm{C} 48$ & $0.31196(16)$ & $0.6326(3)$ & $0.03498(18)$ & $0.0691(10)^{*}$ \\
\hline
\end{tabular}


Table 2. Atomic Coordinates and Displacement Parameters (continued)

(b) solvent dichloromethane atoms

$\begin{array}{lllll}\text { Atom } & x & y & z & U_{\mathrm{eq}}, \AA^{2} \\ \text { C11S } & 0.53886(7) & 0.11383(15) & 0.31736(11) & 0.1460(8)^{*} \\ \text { C1S } & 0.5000 & 0.1950(5) & 0.2500 & 0.0824(17)^{*}\end{array}$

Anisotropically-refined atoms are marked with an asterisk $(*)$. The form of the anisotropic displacement parameter is: $\exp \left[-2 \pi^{2}\left(h^{2} a^{* 2} U_{11}+k^{2} b^{* 2} U_{22}+l^{2} c^{* 2} U_{33}+\right.\right.$ $\left.\left.2 k l b^{*} c^{*} U_{23}+2 h l a^{*} c^{*} U_{13}+2 h k a^{*} b^{*} U_{12}\right)\right]$.

Table 3. Selected Interatomic Distances $(\AA)$

(a) within 1,1,2,2-tetrakis(4-methoxy-2-methylphenyl)ethane

\begin{tabular}{|c|c|c|c|c|c|}
\hline Atom1 & Atom2 & Distance & Atom1 & Atom2 & Distance \\
\hline O1 & C14 & $1.376(3)$ & $\mathrm{C} 21$ & $\mathrm{C} 26$ & $1.388(4)$ \\
\hline O1 & C18 & $1.416(4)$ & $\mathrm{C} 22$ & $\mathrm{C} 23$ & $1.386(4)$ \\
\hline $\mathrm{O} 2$ & $\mathrm{C} 24$ & $1.379(3)$ & $\mathrm{C} 22$ & $\mathrm{C} 27$ & $1.503(4)$ \\
\hline $\mathrm{O} 2$ & $\mathrm{C} 28$ & $1.429(5)$ & $\mathrm{C} 23$ & $\mathrm{C} 24$ & $1.365(4)$ \\
\hline $\mathrm{O} 3$ & C34 & $1.373(4)$ & $\mathrm{C} 24$ & $\mathrm{C} 25$ & $1.406(4)$ \\
\hline $\mathrm{O} 3$ & C38 & $1.425(4)$ & $\mathrm{C} 25$ & $\mathrm{C} 26$ & $1.384(4)$ \\
\hline $\mathrm{O} 4$ & C44 & $1.376(3)$ & C31 & C32 & $1.410(4)$ \\
\hline $\mathrm{O} 4$ & $\mathrm{C} 48$ & $1.407(4)$ & C31 & C36 & $1.385(4)$ \\
\hline $\mathrm{C} 1$ & $\mathrm{C} 2$ & $1.553(3)$ & C32 & C33 & $1.386(4)$ \\
\hline $\mathrm{C} 1$ & $\mathrm{C} 11$ & $1.535(4)$ & C32 & C37 & $1.507(4)$ \\
\hline $\mathrm{C} 1$ & $\mathrm{C} 21$ & $1.524(4)$ & C33 & C34 & $1.372(4)$ \\
\hline $\mathrm{C} 2$ & C31 & $1.525(4)$ & C34 & C35 & $1.379(4)$ \\
\hline $\mathrm{C} 2$ & C41 & $1.539(4)$ & C35 & C36 & $1.391(4)$ \\
\hline $\mathrm{C} 11$ & $\mathrm{C} 12$ & $1.395(4)$ & C41 & $\mathrm{C} 42$ & $1.409(3)$ \\
\hline $\mathrm{C} 11$ & C16 & $1.396(4)$ & C41 & $\mathrm{C} 46$ & $1.384(4)$ \\
\hline $\mathrm{C} 12$ & $\mathrm{C} 13$ & $1.410(4)$ & $\mathrm{C} 42$ & $\mathrm{C} 43$ & $1.379(4)$ \\
\hline $\mathrm{C} 12$ & $\mathrm{C} 17$ & $1.509(4)$ & C42 & $\mathrm{C} 47$ & $1.504(4)$ \\
\hline $\mathrm{C} 13$ & $\mathrm{C} 14$ & $1.376(4)$ & C43 & $\mathrm{C} 44$ & $1.389(4)$ \\
\hline $\mathrm{C} 14$ & $\mathrm{C} 15$ & $1.383(4)$ & C44 & $\mathrm{C} 45$ & $1.378(4)$ \\
\hline $\mathrm{C} 15$ & C16 & $1.370(4)$ & C45 & $\mathrm{C} 46$ & $1.387(4)$ \\
\hline $\mathrm{C} 21$ & $\mathrm{C} 22$ & $1.403(4)$ & & & \\
\hline
\end{tabular}

(b) within the solvent dichloromethane molecule

$\begin{array}{lll}\text { Atom1 } & \text { Atom2 } & \text { Distance } \\ \text { Cl1S } & \text { C1S } & 1.743(4)\end{array}$


Table 4. Selected Interatomic Angles (deg)

(a) within 1,1,2,2-tetrakis (4-methoxy-2-methylphenyl)ethane

\begin{tabular}{|c|c|c|c|c|c|c|c|}
\hline Atom1 & Atom2 & Atom3 & Angle & Atom1 & Atom2 & Atom3 & Angle \\
\hline C14 & $\mathrm{O} 1$ & $\mathrm{C} 18$ & $116.7(3)$ & C33 & C32 & C37 & $118.1(3)$ \\
\hline $\mathrm{C} 24$ & $\mathrm{O} 2$ & $\mathrm{C} 28$ & $115.3(3)$ & $\mathrm{C} 32$ & $\mathrm{C} 33$ & $\mathrm{C} 34$ & $122.3(3)$ \\
\hline C34 & O3 & $\mathrm{C} 38$ & $118.5(3)$ & $\mathrm{O} 3$ & C34 & C33 & $115.4(3)$ \\
\hline C44 & $\mathrm{O} 4$ & $\mathrm{C} 48$ & $117.7(2)$ & $\mathrm{O} 3$ & C34 & C35 & $124.9(3)$ \\
\hline $\mathrm{C} 2$ & $\mathrm{C} 1$ & C11 & $112.0(2)$ & C33 & C34 & C35 & $119.7(3)$ \\
\hline $\mathrm{C} 2$ & $\mathrm{C} 1$ & $\mathrm{C} 21$ & $114.6(2)$ & C34 & $\mathrm{C} 35$ & $\mathrm{C} 36$ & $118.4(3)$ \\
\hline $\mathrm{C} 11$ & $\mathrm{C} 1$ & $\mathrm{C} 21$ & $108.7(2)$ & C31 & C36 & C35 & $123.0(3)$ \\
\hline $\mathrm{C} 1$ & $\mathrm{C} 2$ & $\mathrm{C} 31$ & $110.5(2)$ & $\mathrm{C} 2$ & C41 & C42 & $120.0(2)$ \\
\hline $\mathrm{C} 1$ & $\mathrm{C} 2$ & C41 & $113.5(2)$ & $\mathrm{C} 2$ & C41 & C46 & $122.2(2)$ \\
\hline $\mathrm{C} 31$ & $\mathrm{C} 2$ & C41 & $111.6(2)$ & C42 & C41 & C46 & $117.7(2)$ \\
\hline $\mathrm{C} 1$ & $\mathrm{C} 11$ & $\mathrm{C} 12$ & $123.6(2)$ & $\mathrm{C} 41$ & $\mathrm{C} 42$ & $\mathrm{C} 43$ & $119.0(2)$ \\
\hline $\mathrm{C} 1$ & C11 & $\mathrm{C} 16$ & $118.4(2)$ & C41 & C42 & C47 & $121.7(2)$ \\
\hline $\mathrm{C} 12$ & $\mathrm{C} 11$ & $\mathrm{C} 16$ & $118.0(2)$ & C43 & C42 & C47 & $119.3(2)$ \\
\hline C11 & $\mathrm{C} 12$ & $\mathrm{C} 13$ & $118.9(3)$ & C42 & C43 & C44 & $122.1(2)$ \\
\hline C11 & $\mathrm{C} 12$ & $\mathrm{C} 17$ & 124.1(3) & O4 & C44 & $\mathrm{C} 43$ & $115.8(2)$ \\
\hline $\mathrm{C} 13$ & $\mathrm{C} 12$ & $\mathrm{C} 17$ & $117.0(2)$ & $\mathrm{O} 4$ & C44 & $\mathrm{C} 45$ & $124.8(3)$ \\
\hline $\mathrm{C} 12$ & $\mathrm{C} 13$ & C14 & $121.3(3)$ & $\mathrm{C} 43$ & C44 & $\mathrm{C} 45$ & $119.4(3)$ \\
\hline $\mathrm{O} 1$ & C14 & $\mathrm{C} 13$ & $125.4(3)$ & $\mathrm{C} 44$ & $\mathrm{C} 45$ & $\mathrm{C} 46$ & $118.5(3)$ \\
\hline $\mathrm{O} 1$ & C14 & $\mathrm{C} 15$ & $114.9(3)$ & $\mathrm{C} 41$ & $\mathrm{C} 46$ & $\mathrm{C} 45$ & $123.2(2)$ \\
\hline $\mathrm{C} 13$ & $\mathrm{C} 14$ & $\mathrm{C} 15$ & $119.7(3)$ & & & & \\
\hline C14 & $\mathrm{C} 15$ & $\mathrm{C} 16$ & $119.2(3)$ & & & & \\
\hline C11 & C16 & $\mathrm{C} 15$ & $122.8(3)$ & & & & \\
\hline $\mathrm{C} 1$ & $\mathrm{C} 21$ & $\mathrm{C} 22$ & $119.3(2)$ & & & & \\
\hline $\mathrm{C} 1$ & $\mathrm{C} 21$ & $\mathrm{C} 26$ & $122.2(2)$ & & & & \\
\hline $\mathrm{C} 22$ & $\mathrm{C} 21$ & $\mathrm{C} 26$ & $118.4(2)$ & & & & \\
\hline $\mathrm{C} 21$ & $\mathrm{C} 22$ & $\mathrm{C} 23$ & $119.8(3)$ & & & & \\
\hline $\mathrm{C} 21$ & $\mathrm{C} 22$ & $\mathrm{C} 27$ & $121.5(3)$ & & & & \\
\hline $\mathrm{C} 23$ & $\mathrm{C} 22$ & $\mathrm{C} 27$ & $118.7(3)$ & & & & \\
\hline $\mathrm{C} 22$ & $\mathrm{C} 23$ & $\mathrm{C} 24$ & $121.3(3)$ & & & & \\
\hline $\mathrm{O} 2$ & $\mathrm{C} 24$ & $\mathrm{C} 23$ & $125.0(3)$ & & & & \\
\hline $\mathrm{O} 2$ & $\mathrm{C} 24$ & $\mathrm{C} 25$ & $114.9(3)$ & & & & \\
\hline $\mathrm{C} 23$ & $\mathrm{C} 24$ & $\mathrm{C} 25$ & $120.0(3)$ & & & & \\
\hline $\mathrm{C} 24$ & $\mathrm{C} 25$ & $\mathrm{C} 26$ & $118.6(3)$ & & & & \\
\hline $\mathrm{C} 21$ & $\mathrm{C} 26$ & $\mathrm{C} 25$ & $122.0(3)$ & & & & \\
\hline $\mathrm{C} 2$ & $\mathrm{C} 31$ & $\mathrm{C} 32$ & $122.8(2)$ & & & & \\
\hline $\mathrm{C} 2$ & C31 & $\mathrm{C} 36$ & $119.6(2)$ & & & & \\
\hline $\mathrm{C} 32$ & C31 & $\mathrm{C} 36$ & $117.6(2)$ & & & & \\
\hline $\mathrm{C} 31$ & $\mathrm{C} 32$ & $\mathrm{C} 33$ & $118.9(3)$ & & & & \\
\hline $\mathrm{C} 31$ & C32 & C37 & $123.0(2)$ & & & & \\
\hline
\end{tabular}


(b) within the solvent dichloromethane molecule

Atom1 Atom2 Atom3 Angle

Cl1S C1S C11S' 113.7(4)

Primed atoms are related to unprimed ones via the crystallographic twofold axis $(1 / 2,1 / 2$, $1 / 4)$.

Table 5. Anisotropic Displacement Parameters $\left(U_{\mathrm{ij}}, \AA^{2}\right)$

\begin{tabular}{|c|c|c|c|c|c|c|}
\hline tom & $U_{11}$ & $U_{22}$ & $U_{33}$ & $U_{23}$ & $U_{13}$ & $U_{12}$ \\
\hline 1 & 15) & 054 & 64 & 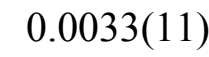 & 0.029 & \\
\hline 2 & & & & & & \\
\hline 3 & $(12)$ & $.0697(16)$ & $.097(2)$ & 0.0 & 0.0 & 0. \\
\hline 4 & & & & & & \\
\hline 1 & & 2) & & -0.0032( & & \\
\hline 2 & & & & & & \\
\hline 11 & & 4) & & -0.0 & 0.0 & $0 .($ \\
\hline 12 & & & & & & \\
\hline 13 & & & & $-0 .($ & & $-0 .($ \\
\hline & & & & & & \\
\hline 15 & & & & -0. & & -0. \\
\hline 16 & & & & & & \\
\hline 17 & & & & & & \\
\hline 18 & & & & & & -0. \\
\hline & & & & & & -0. \\
\hline 22 & & & & & & -0 . \\
\hline & & & & & & -0 . \\
\hline 24 & & & & & -0 . & $-0 .($ \\
\hline & & & & & & \\
\hline 26 & & & & -0. & & $-0 .($ \\
\hline & & & & & & -0.0 \\
\hline 0 & & & & -0. & $-0 .($ & -0.0 \\
\hline & & & & & & \\
\hline- & & 0.0 & & & 0.0 & -0.00 \\
\hline & & & & & & -0.0 \\
\hline 34 & & & & & & (13) \\
\hline & & & & & & \\
\hline $\mathrm{C} 36$ & $0.0428(15)$ & $0.0353(14)$ & $0.0453(15)$ & $0.0005(11)$ & $0.0144(12)$ & $-0.0002(11$ \\
\hline
\end{tabular}




$\begin{array}{lllllll}\text { C37 } & 0.0499(17) & 0.0381(15) & 0.0559(18) & -0.0064(13) & 0.0001(14) & -0.0040(13) \\ \text { C38 } & 0.0497(19) & 0.083(3) & 0.090(3) & 0.014(2) & 0.0167(19) & 0.0225(19) \\ \text { C41 } & 0.0339(13) & 0.0357(13) & 0.0307(12) & -0.0011(10) & 0.0073(10) & 0.0055(10) \\ \text { C42 } & 0.0344(13) & 0.0412(14) & 0.0310(13) & -0.0038(11) & 0.0062(10) & 0.0059(11) \\ \text { C43 } & 0.0423(15) & 0.0520(16) & 0.0287(13) & -0.0013(11) & 0.0084(11) & 0.0040(12) \\ \text { C44 } & 0.0394(14) & 0.0446(15) & 0.0365(14) & 0.0087(12) & 0.0084(11) & 0.0051(12) \\ \text { C45 } & 0.0472(15) & 0.0372(14) & 0.0340(14) & 0.0002(11) & 0.0042(12) & -0.0015(12) \\ \text { C46 } & 0.0493(15) & 0.0400(14) & 0.0283(12) & -0.0018(11) & 0.0088(11) & -0.0009(12) \\ \text { C47 } & 0.0500(17) & 0.0589(19) & 0.0357(15) & -0.0132(13) & 0.0119(13) & -0.0052(14) \\ \text { C48 } & 0.070(2) & 0.080(2) & 0.053(2) & 0.0096(18) & 0.0104(17) & -0.0292(19) \\ \text { C11S } & 0.1270(12) & 0.1385(13) & 0.2007(18) & 0.0967(13) & 0.0928(13) & 0.0631(10) \\ \text { C1S } & 0.089(4) & 0.060(3) & 0.104(5) & 0.000 & 0.036(4) & 0.000\end{array}$

The form of the anisotropic displacement parameter is:

$\exp \left[-2 \pi^{2}\left(h^{2} a^{* 2} U_{11}+k^{2} b^{* 2} U_{22}+l^{2} c^{* 2} U_{33}+2 k l b^{*} c^{*} U_{23}+2 h l a^{*} c^{*} U_{13}+2 h k a^{*} b^{*} U_{12}\right)\right]$ 
Table 6. Derived Atomic Coordinates and Displacement Parameters for Hydrogen Atoms

$\begin{array}{lcccc}\text { Atom } & x & y & z & U_{\text {eq }} \AA^{2} \\ \text { H1 } & 0.1640 & 0.3342 & 0.2169 & 0.041 \\ \text { H2 } & 0.1618 & 0.1761 & 0.1097 & 0.041 \\ \text { H13 } & 0.0472 & 0.1325 & 0.3137 & 0.053 \\ \text { H15 } & 0.1455 & -0.1046 & 0.2646 & 0.052 \\ \text { H16 } & 0.1867 & 0.0353 & 0.2165 & 0.046 \\ \text { H17A } & 0.1104 & 0.3719 & 0.2783 & 0.065 \\ \text { H17B } & 0.0584 & 0.3232 & 0.3002 & 0.065 \\ \text { H17C } & 0.0548 & 0.3396 & 0.2218 & 0.065 \\ \text { H18A } & 0.0210 & -0.1305 & 0.3770 & 0.078 \\ \text { H18B } & 0.0016 & -0.0201 & 0.3310 & 0.078 \\ \text { H18C } & 0.0522 & -0.0117 & 0.3978 & 0.078 \\ \text { H23 } & 0.3323 & 0.2837 & 0.3728 & 0.056 \\ \text { H25 } & 0.3430 & 0.1118 & 0.2062 & 0.056 \\ \text { H26 } & 0.2503 & 0.1518 & 0.1549 & 0.049 \\ \text { H27A } & 0.1991 & 0.3061 & 0.3446 & 0.065 \\ \text { H27B } & 0.2138 & 0.4188 & 0.3094 & 0.065 \\ \text { H27C } & 0.2542 & 0.3756 & 0.3794 & 0.065 \\ \text { H28A } & 0.4649 & 0.1846 & 0.4029 & 0.095 \\ \text { H28B } & 0.4067 & 0.1749 & 0.4198 & 0.095 \\ \text { H28C } & 0.4235 & 0.2908 & 0.3896 & 0.095 \\ \text { H33 } & -0.0269 & 0.1411 & 0.0424 & 0.060 \\ \text { H35 } & -0.0009 & 0.4754 & 0.0813 & 0.058 \\ \text { H36 } & 0.0939 & 0.4414 & 0.1128 & 0.049 \\ \text { H37A } & 0.0917 & 0.0311 & 0.1164 & 0.061 \\ \text { H37B } & 0.0985 & 0.0572 & 0.0427 & 0.061 \\ \text { H37C } & 0.0408 & 0.0115 & 0.0509 & 0.061 \\ \text { H38A } & -0.1443 & 0.4185 & 0.0300 & 0.089 \\ \text { H38B } & -0.0925 & 0.4772 & 0.0124 & 0.089 \\ \text { H38C } & -0.0920 & 0.4577 & 0.0896 & 0.089 \\ \text { H43 } & 0.2168 & 0.3843 & -0.0508 & 0.050 \\ \text { H45 } & 0.2627 & 0.5679 & 0.1216 & 0.049 \\ \text { H46 } & 0.2114 & 0.4455 & 0.1695 & 0.047 \\ \text { H47A } & 0.1608 & 0.2283 & -0.0645 & 0.058 \\ \text { H47B } & 0.1140 & 0.2390 & -0.0255 & 0.058 \\ \text { H47C } & 0.1651 & 0.1546 & 0.0022 & 0.058 \\ \text { H48A } & 0.3313 & 0.6706 & 0.0056 & 0.083 \\ \text { H48B } & 0.3390 & 0.5975 & 0.0733 & 0.083 \\ \text { H48C } & 0.2901 & 0.6886 & 0.0518 & 0.083 \\ \text { H1SB } a & 0.4748 & 0.2447 & 0.2666 & 0.099 \\ \text { H1S } & 0.5252 & 0.2447 & 0.2334 & 0.099\end{array}$

$a$ Included with an occupancy factor of 0.5 . 


\section{STRUCTURE REPORT}

Compound: $9,10-\mathrm{Bis}(o$-tolyl)anthracene, chloroform solvate 6

Formula: $\quad \mathrm{C}_{29} \mathrm{H}_{23} \mathrm{Cl}_{3}\left(\mathrm{C}_{28} \mathrm{H}_{22} \cdot \mathrm{CHCl}_{3}\right)$

Crystallographer: R. McDonald

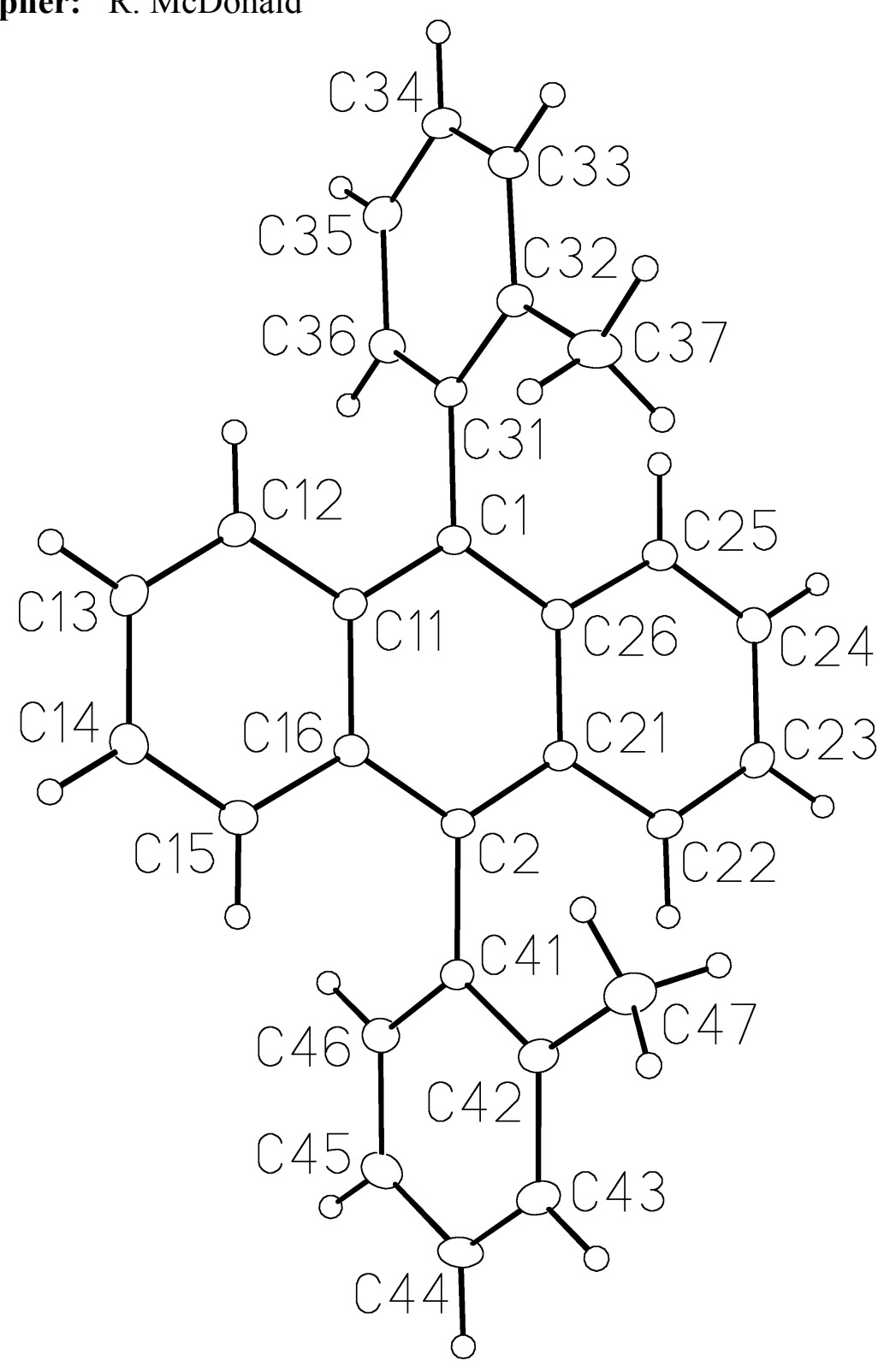

Figure 1. Perspective view of the 9,10-bis(o-tolyl)anthracene molecule showing the atom labelling scheme. Non-hydrogen atoms are represented by Gaussian ellipsoids at the $20 \%$ probability level. Hydrogen atoms are shown with arbitrarily small thermal parameters. 


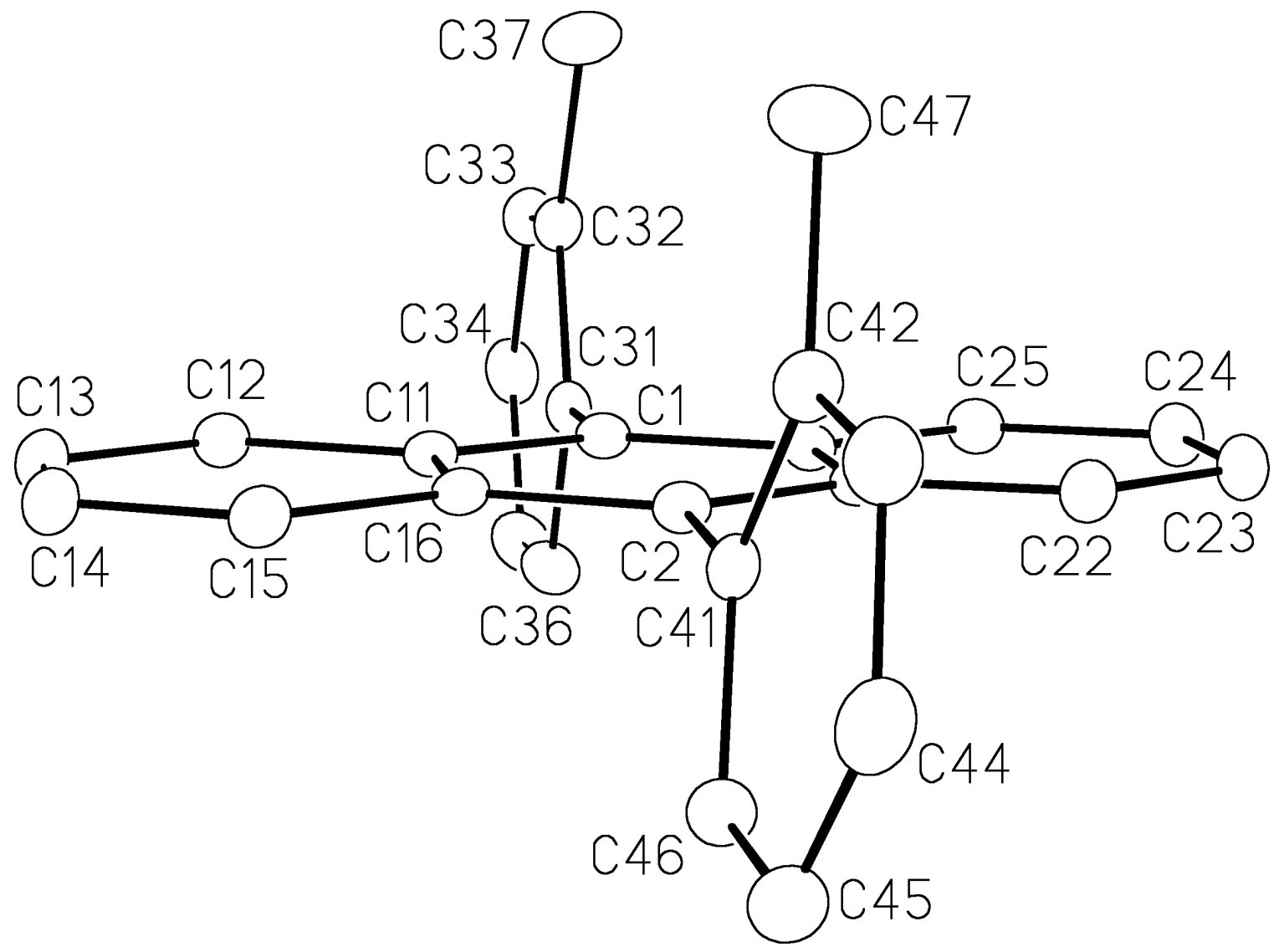

Figure 2. Illustration of the near-orthoganol relative orientations of the $o$-tolyl groups with respect to the anthracene group. Hydrogen atoms are not shown. 


\section{List of Tables}

Table 1. Crystallographic Experimental Details

Table 2. Atomic Coordinates and Equivalent Isotropic Displacement Parameters

Table 3. Selected Interatomic Distances

Table 4. Selected Interatomic Angles

Table 5. Anisotropic Displacement Parameters

Table 6. Derived Atomic Coordinates and Displacement Parameters for Hydrogen Atoms

Table 1. Crystallographic Experimental Details

$\begin{array}{ll}\begin{array}{l}\text { A. Crystal Data } \\ \text { formula }\end{array} & \\ \text { formula weight } & \mathrm{C}_{29} \mathrm{H}_{23} \mathrm{Cl}_{3} \\ \text { crystal dimensions }(\mathrm{mm}) & 477.82 \\ \text { crystal system } & 0.47 \times 0.45 \times \\ \text { space group } & \text { monoclinic } \\ \text { unit cell parameters }{ }^{a} & I 2 / a(\text { an altern } \\ \quad a(\AA) & \\ \quad b(\AA) & 18.1743(16) \\ \quad c(\AA) & 14.9401(13) \\ \quad \beta(\mathrm{deg}) & 17.2589(15) \\ \quad V\left(\AA \AA^{3}\right) & 95.7046(12) \\ \quad Z & 4663.0(7) \\ \rho_{\text {calcd }}\left(\mathrm{g} \mathrm{cm}^{-3}\right) & 8 \\ \mu\left(\mathrm{mm}^{-1}\right) & 1.361\end{array}$

B. Data Collection and Refinement Conditions diffractometer radiation $(\lambda[\AA])$

temperature $\left({ }^{\circ} \mathrm{C}\right)$

scan type

data collection $2 \theta$ limit (deg)

total data collected
Bruker PLATFORM/SMART $1000 \mathrm{CCD}^{b}$ graphite-monochromated Mo K $\alpha(0.71073)$ $-80$ $\omega$ scans $\left(0.4^{\circ}\right)(10 \mathrm{~s}$ exposures $)$ 52.86 $15229(-22 \leq h \leq 22,-17 \leq k \leq 18,-21 \leq l \leq$ 21) 
independent reflections

number of observed reflections $(\mathrm{NO})$

structure solution method

refinement method

(SHELXL-93d)

absorption correction method

range of transmission factors

data/restraints/parameters

goodness-of-fit $(S)^{e}$

final $R$ indices $f$

$$
R_{1}\left[F_{\mathrm{o}}^{2} \geq 2 \sigma\left(F_{\mathrm{o}}^{2}\right)\right]
$$$$
w R_{2}\left[F_{\mathrm{o}}^{2} \geq-3 \sigma\left(F_{\mathrm{o}}^{2}\right)\right]
$$

largest difference peak and hole
$4792\left(R_{\text {int }}=0.0316\right)$

$3645\left[F_{\mathrm{o}}^{2} \geq 2 \sigma\left(F_{\mathrm{o}}^{2}\right)\right]$

direct methods (SHELXS-86c)

full-matrix least-squares on $F^{2}$

multi-scan (SADABS)

0.8736-0.8311

$4792\left[F_{\mathrm{o}}^{2} \geq-3 \sigma\left(F_{\mathrm{o}}^{2}\right)\right] / 0 / 291$

$1.026\left[F_{\mathrm{o}}^{2} \geq-3 \sigma\left(F_{\mathrm{o}}^{2}\right)\right]$

${ }^{a}$ Obtained from least-squares refinement of 5833 reflections with $4.50^{\circ}<2 \theta<52.70^{\circ}$.

$b$ Programs for diffractometer operation, data collection, data reduction and absorption correction were those supplied by Bruker.

cSheldrick, G. M. Acta Crystallogr. 1990, A46, 467-473.

dSheldrick, G. M. SHELXL-93. Program for crystal structure determination. University of Göttingen, Germany, 1993.

$e S=\left[\Sigma w\left(F_{\mathrm{o}}^{2}-F_{\mathrm{c}}\right)^{2} /(n-p)\right]^{1 / 2}(n=$ number of data; $p=$ number of parameters varied; $w$ $=\left[\sigma^{2}\left(F_{\mathrm{o}}^{2}\right)+(0.0520 P)^{2}+4.0971 P\right]^{-1}$ where $\left.P=\left[\operatorname{Max}\left(F_{\mathrm{o}}^{2}, 0\right)+2 F_{\mathrm{c}}^{2}\right] / 3\right)$.

$f_{R_{1}}=\Sigma|| F_{\mathrm{o}}|-| F_{\mathrm{c}}|| / \Sigma\left|F_{\mathrm{o}}\right| ; w R_{2}=\left[\Sigma w\left(F_{\mathrm{o}}^{2}-F_{\mathrm{c}}^{2}\right)^{2} / \Sigma w\left(F_{\mathrm{o}}{ }^{4}\right)\right]^{1 / 2}$. 
Table 2. Atomic Coordinates and Equivalent Isotropic Displacement Parameters

(a) atoms of 9,10-bis(o-tolyl)anthracene

$\begin{array}{lclll}\text { Atom } & x & y & z & U_{\text {eq }} \AA^{2} \\ \text { C1 } & -0.04589(9) & 0.29839(12) & -0.00493(10) & 0.0248(4)^{*} \\ \text { C2 } & 0.06837(9) & 0.18712(11) & 0.06645(10) & 0.0242(4)^{*} \\ \text { C11 } & -0.05945(9) & 0.24350(12) & 0.05815(10) & 0.0246(4)^{*} \\ \text { C12 } & -0.12987(10) & 0.24168(13) & 0.08861(11) & 0.0304(4)^{*} \\ \text { C13 } & -0.14179(10) & 0.18916(13) & 0.15019(11) & 0.0346(4)^{*} \\ \text { C14 } & -0.08474(11) & 0.13494(13) & 0.18651(12) & 0.0343(4)^{*} \\ \text { C15 } & -0.01710(10) & 0.13396(12) & 0.15950(11) & 0.0302(4)^{*} \\ \text { C16 } & -0.00150(9) & 0.18754(11) & 0.09460(10) & 0.0245(4)^{*} \\ \text { C21 } & 0.08130(9) & 0.24050(12) & 0.00223(10) & 0.0248(4)^{*} \\ \text { C22 } & 0.15110(9) & 0.24051(13) & -0.02891(11) & 0.0296(4)^{*} \\ \text { C23 } & 0.16352(10) & 0.29239(14) & -0.09089(11) & 0.0341(4)^{*} \\ \text { C24 } & 0.10690(10) & 0.34799(13) & -0.12609(11) & 0.0338(4)^{*} \\ \text { C25 } & 0.03988(10) & 0.35046(12) & -0.09850(11) & 0.0299(4)^{*} \\ \text { C26 } & 0.02382(9) & 0.29723(12) & -0.03362(10) & 0.0248(4)^{*} \\ \text { C31 } & -0.10544(9) & 0.35888(12) & -0.04166(10) & 0.0264(4)^{*} \\ \text { C32 } & -0.15349(10) & 0.32977(13) & -0.10494(11) & 0.0302(4)^{*} \\ \text { C33 } & -0.20788(10) & 0.38849(14) & -0.13662(11) & 0.0360(5)^{*} \\ \text { C34 } & -0.21467(11) & 0.47339(15) & -0.10715(12) & 0.0392(5)^{*} \\ \text { C35 } & -0.16713(12) & 0.50188(14) & -0.04529(12) & 0.0408(5)^{*} \\ \text { C36 } & -0.11231(11) & 0.44489(13) & -0.01261(11) & 0.0350(4)^{*} \\ \text { C37 } & -0.14607(12) & 0.23812(14) & -0.13834(14) & 0.0459(5)^{*} \\ \text { C41 } & 0.13063(9) & 0.13328(12) & 0.10656(10) & 0.0257(4)^{*} \\ \text { C42 } & 0.15269(10) & 0.05137(13) & 0.07701(12) & 0.0324(4)^{*} \\ \text { C43 } & 0.21456(11) & 0.00903(14) & 0.11505(13) & 0.0386(5)^{*} \\ \text { C44 } & 0.25281(11) & 0.04468(15) & 0.18024(13) & 0.0414(5)^{*} \\ \text { C45 } & 0.23006(11) & 0.12434(15) & 0.21042(12) & 0.0408(5)^{*} \\ \text { C46 } & 0.16933(10) & 0.16804(14) & 0.17336(11) & 0.0338(4)^{*} \\ \text { C47 } & 0.11228(13) & 0.00958(16) & 0.00587(14) & 0.0505(6)^{*} \\ & & & & \end{array}$

(b) solvent chloroform atoms

$\begin{array}{lllll}\text { Atom } & x & y & z & U_{\text {eq }}, \AA^{2} \\ \text { Cl1S } & 0.44865(4) & 0.07987(4) & 0.33571(3) & 0.05406(18)^{*} \\ \text { Cl2S } & 0.58918(3) & 0.06458(4) & 0.27161(4) & 0.05519(18)^{*} \\ \text { Cl3S } & 0.46056(4) & 0.12387(5) & 0.17551(4) & 0.0653(2)^{*} \\ \text { C1S } & 0.50621(11) & 0.12476(14) & 0.26961(12) & 0.0381(5)^{*}\end{array}$

Anisotropically-refined atoms are marked with an asterisk $\left(^{*}\right)$. The form of the anisotropic displacement parameter is: $\exp \left[-2 \pi^{2}\left(h^{2} a^{* 2} U_{11}+k^{2} b^{* 2} U_{22}+l^{2} c^{* 2} U_{33}+\right.\right.$ $\left.\left.2 k l b^{*} c^{*} U_{23}+2 h l a^{*} c^{*} U_{13}+2 h k a^{*} b^{*} U_{12}\right)\right]$. 
Table 3. Selected Interatomic Distances $(\AA)$

(a) within 9,10-bis(o-tolyl)anthracene

$\begin{array}{llllll}\text { Atom1 } & \text { Atom2 } & \text { Distance } & \text { Atom1 } & \text { Atom2 } & \text { Distance } \\ \mathrm{C} 1 & \mathrm{C} 11 & 1.404(2) & \mathrm{C} 24 & \mathrm{C} 25 & 1.352(3) \\ \mathrm{C} 1 & \mathrm{C} 26 & 1.405(2) & \mathrm{C} 25 & \mathrm{C} 26 & 1.427(3) \\ \mathrm{C} 1 & \mathrm{C} 31 & 1.501(2) & \mathrm{C} 31 & \mathrm{C} 32 & 1.398(3) \\ \mathrm{C} 2 & \mathrm{C} 16 & 1.404(2) & \mathrm{C} 31 & \mathrm{C} 36 & 1.389(3) \\ \mathrm{C} 2 & \mathrm{C} 21 & 1.404(2) & \mathrm{C} 32 & \mathrm{C} 33 & 1.392(3) \\ \mathrm{C} 2 & \mathrm{C} 41 & 1.500(2) & \mathrm{C} 32 & \mathrm{C} 37 & 1.497(3) \\ \mathrm{C} 11 & \mathrm{C} 12 & 1.431(2) & \mathrm{C} 33 & \mathrm{C} 34 & 1.377(3) \\ \mathrm{C} 11 & \mathrm{C} 16 & 1.440(2) & \mathrm{C} 34 & \mathrm{C} 35 & 1.373(3) \\ \mathrm{C} 12 & \mathrm{C} 13 & 1.356(3) & \mathrm{C} 35 & \mathrm{C} 36 & 1.387(3) \\ \mathrm{C} 13 & \mathrm{C} 14 & 1.412(3) & \mathrm{C} 41 & \mathrm{C} 42 & 1.400(3) \\ \mathrm{C} 14 & \mathrm{C} 15 & 1.358(3) & \mathrm{C} 41 & \mathrm{C} 46 & 1.390(3) \\ \mathrm{C} 15 & \mathrm{C} 16 & 1.428(2) & \mathrm{C} 42 & \mathrm{C} 43 & 1.396(3) \\ \mathrm{C} 21 & \mathrm{C} 22 & 1.426(2) & \mathrm{C} 42 & \mathrm{C} 47 & 1.502(3) \\ \mathrm{C} 21 & \mathrm{C} 26 & 1.437(2) & \mathrm{C} 43 & \mathrm{C} 44 & 1.370(3) \\ \mathrm{C} 22 & \mathrm{C} 23 & 1.358(3) & \mathrm{C} 44 & \mathrm{C} 45 & 1.379(3) \\ \mathrm{C} 23 & \mathrm{C} 24 & 1.412(3) & \mathrm{C} 45 & \mathrm{C} 46 & 1.383(3) \\ \text { (b) within } & \text { the solvent } \text { chloroform molecule } & & & \\ \text { Atom1 } & \text { Atom2 } & \text { Distance } & \text { Atom1 } & \text { Atom2 } & \text { Distance } \\ \text { C11S } & \mathrm{C} 1 \mathrm{~S} & 1.756(2) & \mathrm{C} 13 \mathrm{~S} & \mathrm{C} 1 \mathrm{~S} & 1.749(2) \\ \mathrm{C} 12 \mathrm{~S} & \mathrm{C} 1 \mathrm{~S} & 1.753(2) & & & \end{array}$


Table 4. Selected Interatomic Angles (deg)

(a) within 9,10-bis(o-tolyl)anthracene

$\begin{array}{llllllll}\text { Atom } 1 & \text { Atom2 } & \text { Atom3 } & \text { Angle } & \text { Atom1 } & \text { Atom2 } & \text { Atom3 } & \text { Angle } \\ \text { C11 } & \text { C1 } & \text { C26 } & 119.98(15) & \text { C1 } & \text { C26 } & \text { C21 } & 119.86(16) \\ \text { C11 } & \text { C1 } & \text { C31 } & 120.28(15) & \text { C1 } & \text { C26 } & \text { C25 } & 122.11(16) \\ \text { C26 } & \text { C1 } & \text { C31 } & 119.73(16) & \text { C21 } & \text { C26 } & \text { C25 } & 118.03(16) \\ \text { C16 } & \text { C2 } & \text { C21 } & 119.92(15) & \text { C1 } & \text { C31 } & \text { C32 } & 120.71(16) \\ \text { C16 } & \text { C2 } & \text { C41 } & 120.61(15) & \text { C1 } & \text { C31 } & \text { C36 } & 119.42(16) \\ \text { C21 } & \text { C2 } & \text { C41 } & 119.41(15) & \text { C32 } & \text { C31 } & \text { C36 } & 119.86(17) \\ \text { C1 } & \text { C11 } & \text { C12 } & 121.87(16) & \text { C31 } & \text { C32 } & \text { C33 } & 118.34(18) \\ \text { C1 } & \text { C11 } & \text { C16 } & 120.05(15) & \text { C31 } & \text { C32 } & \text { C37 } & 120.80(17) \\ \text { C12 } & \text { C11 } & \text { C16 } & 118.07(16) & \text { C33 } & \text { C32 } & \text { C37 } & 120.85(17) \\ \text { C11 } & \text { C12 } & \text { C13 } & 121.15(17) & \text { C32 } & \text { C33 } & \text { C34 } & 121.45(18) \\ \text { C12 } & \text { C13 } & \text { C14 } & 120.83(18) & \text { C33 } & \text { C34 } & \text { C35 } & 120.03(18) \\ \text { C13 } & \text { C14 } & \text { C15 } & 120.24(18) & \text { C34 } & \text { C35 } & \text { C36 } & 119.8(2) \\ \text { C14 } & \text { C15 } & \text { C16 } & 121.37(17) & \text { C31 } & \text { C36 } & \text { C35 } & 120.51(19) \\ \text { C2 } & \text { C16 } & \text { C11 } & 119.93(16) & \text { C2 } & \text { C41 } & \text { C42 } & 122.06(16) \\ \text { C2 } & \text { C16 } & \text { C15 } & 121.73(16) & \text { C2 } & \text { C41 } & \text { C46 } & 118.59(16) \\ \text { C11 } & \text { C16 } & \text { C15 } & 118.34(16) & \text { C42 } & \text { C41 } & \text { C46 } & 119.29(16) \\ \text { C2 } & \text { C21 } & \text { C22 } & 121.49(16) & \text { C41 } & \text { C42 } & \text { C43 } & 118.12(18) \\ \text { C2 } & \text { C21 } & \text { C26 } & 120.23(15) & \text { C41 } & \text { C42 } & \text { C47 } & 121.78(17) \\ \text { C22 } & \text { C21 } & \text { C26 } & 118.28(16) & \text { C43 } & \text { C42 } & \text { C47 } & 120.10(18) \\ \text { C21 } & \text { C22 } & \text { C23 } & 121.37(16) & \text { C42 } & \text { C43 } & \text { C44 } & 121.95(19) \\ \text { C22 } & \text { C23 } & \text { C24 } & 120.24(17) & \text { C43 } & \text { C44 } & \text { C45 } & 119.93(18) \\ \text { C23 } & \text { C24 } & \text { C25 } & 120.54(18) & \text { C44 } & \text { C45 } & \text { C46 } & 119.22(19) \\ \text { C24 } & \text { C25 } & \text { C26 } & 121.54(17) & \text { C41 } & \text { C46 } & \text { C45 } & 121.46(19)\end{array}$

(b) within the solvent chloroform molecule

Atom1 Atom2 Atom3 Angle Atom1 Atom2 Atom3 Angle

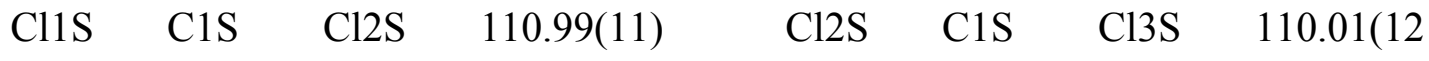

Cl1S C1S Cl3S 110.01(11) 
Table 5. Anisotropic Displacement Parameters $\left(U_{\mathrm{ij}}, \AA^{2}\right)$

\begin{tabular}{|c|c|c|c|c|c|c|}
\hline Atom & $U_{11}$ & $U_{22}$ & $U_{33}$ & $U_{23}$ & $U_{13}$ & $U_{12}$ \\
\hline $\mathrm{C} 1$ & $0.0239(8)$ & $0.0245(9)$ & $0.0250(9)$ & $-0.0026(7)$ & $-0.0022(7)$ & $.0030(7)$ \\
\hline $\mathrm{C} 2$ & $0.0243(8)$ & $0.0216(9)$ & $.0260(9)$ & $-0.0008(7)$ & $-0.0009(7)$ & $0.0024(7)$ \\
\hline $\mathrm{C} 11$ & $0.0235(8)$ & $0.0234(9)$ & $0.0263(9)$ & $-0.0043(7)$ & $-0.0001(7)$ & $0.0006(7)$ \\
\hline $\mathrm{C} 12$ & $0.0250(9)$ & $0.0324(10)$ & $0.0335(10)$ & $-0.0026(8)$ & $0.0014(7)$ & $0.0016(7)$ \\
\hline $\mathrm{C} 13$ & $0.0271(9)$ & $0.0375(11)$ & $0.0403(11)$ & $-0.0003(9)$ & 0.008 & $-0.0026(8)$ \\
\hline $\mathrm{C} 14$ & $0.0361(10)$ & $0.0332(11)$ & $0.0342(10)$ & $0.0065(8)$ & $0.0056(8)$ & $-0.0043(8)$ \\
\hline 15 & $0.0305(9)$ & $0.0266(10)$ & $0.0329(10)$ & $0.0028(8)$ & & $0.0007(7)$ \\
\hline $\mathrm{C} 16$ & $0.0254(8)$ & $0.0214(9)$ & $0.0259(9)$ & $-0.0025(7)$ & $-0.0012(7)$ & $-0.0010(7)$ \\
\hline $\mathrm{C} 21$ & $0.0230(8)$ & 0.02 & 0.02 & $-0.0028(7)$ & $-0.0005(7)$ & $0.0009(7)$ \\
\hline $\mathrm{C} 22$ & $0.0237(9)$ & $0.0309(10)$ & $0.0339(10)$ & $0.0022(8)$ & $0.0020(7)$ & $0.0045(7)$ \\
\hline $\mathrm{C} 23$ & $0.0281(9)$ & $0.0376(11)$ & $0.0377(11)$ & $0.0009(9)$ & $0.0094(8)$ & $7(8)$ \\
\hline $\mathrm{C} 24$ & 0.03 & 0.03 & 0.03 & $0.0067(8)$ & $0.0078(8)$ & 0.00 \\
\hline $\mathrm{C} 25$ & 0.03 & 0.02 & & & & 0.0 \\
\hline $\mathrm{C} 26$ & $0.0260(8)$ & 0.02 & $0.0251(9)$ & $-0.0021(7)$ & $0.0010(7)$ & $0.0018(7)$ \\
\hline C31 & $0.0227(8)$ & $0.0296(10)$ & $0.0272(9)$ & $0.0040(7)$ & $0.0040(7)$ & $36(7)$ \\
\hline $\mathrm{C} 32$ & 0.02 & 0.0 & $0.0316(10)$ & $0.0024(8)$ & $0.0023(7)$ & $7(7)$ \\
\hline C33 & & & & & & \\
\hline 34 & $0.0330(10)$ & (12) & 0.0 & 0.0 & $0.0053(9)$ & $3(9)$ \\
\hline $\mathrm{C} 35$ & $5(12)$ & (11) & $3(12)$ & $0.0011(9)$ & $0.0073(10)$ & $4(9)$ \\
\hline C36 & $0(10)$ & (11) & (10) & $-0.0013(8)$ & $0.0009(8)$ & $6(8)$ \\
\hline C37 & 0.04 & & & & & \\
\hline C41 & $0.0213(8)$ & $0.0273(10)$ & $0.0290(9)$ & 0.00 & $0.0041(7)$ & 1(7) \\
\hline $\mathrm{C} 42$ & 0.02 & $0.0294(10)$ & $3(10)$ & $0.0029(8)$ & $0.0023(8)$ & $0(8)$ \\
\hline $\mathrm{C} 43$ & $8(10)$ & (11) & $4(12)$ & $0.0060(9)$ & $0.0054(9)$ & $0.0093(8)$ \\
\hline $\mathrm{C} 44$ & $0.0279(10)$ & 0.0 & $0(12)$ & $7(10)$ & $-0.0024(9)$ & $0.0078(9)$ \\
\hline $\mathrm{C} 45$ & $0.0335(10)$ & 0.0 & 0.0 & $4(10)$ & $-0.0064(8)$ & $0.0003(9)$ \\
\hline $\mathrm{C} 46$ & $0.0309(9)$ & (11) & (10) & $0.0003(8)$ & $-0.0004(8)$ & $0.0020(8)$ \\
\hline $\mathrm{C} 47$ & $0.0521(13)$ & $0.0411(13)$ & $0.0553(14)$ & $-0.0150(11)$ & $-0.0098(11)$ & $0.0121(10)$ \\
\hline $\mathrm{Cl1S}$ & $0.0625(4)$ & $0.0540(4)$ & $0.0469(3)$ & $-0.0006(3)$ & $0.0116(3)$ & $-0.0102(3)$ \\
\hline $\mathrm{Cl2S}$ & $0.0455(3)$ & $0.0428(3)$ & $0.0756(4)$ & $0.0106(3)$ & $-0.0023(3)$ & $0.0035(2)$ \\
\hline $\mathrm{Cl3S}$ & $0.0680(4)$ & $0.0739(5)$ & $0.0485(3)$ & $0.0238(3)$ & $-0.0209(3)$ & $-0.0165(3)$ \\
\hline C1S & $0.0443(11)$ & $0.0294(11)$ & $0.0388(11)$ & $0.0008(9)$ & $-0.0053(9)$ & $-0.0032(9)$ \\
\hline
\end{tabular}

The form of the anisotropic displacement parameter is:

$\exp \left[-2 \pi^{2}\left(h^{2} a^{* 2} U_{11}+k^{2} b^{* 2} U_{22}+l^{2} c^{* 2} U_{33}+2 k l b^{*} c^{*} U_{23}+2 h l a^{*} c^{*} U_{13}+2 h k a^{*} b^{*} U_{12}\right)\right]$ 
Table 6. Derived Atomic Coordinates and Displacement Parameters for Hydrogen Atoms

$\begin{array}{lcccc}\text { Atom } & x & y & z & U_{\text {eq }}, \AA^{2} \\ \text { H12 } & -0.1689 & 0.2779 & 0.0653 & 0.036 \\ \text { H13 } & -0.1891 & 0.1888 & 0.1691 & 0.042 \\ \text { H14 } & -0.0937 & 0.0990 & 0.2300 & 0.041 \\ \text { H15 } & 0.0206 & 0.0969 & 0.1843 & 0.036 \\ \text { H22 } & 0.1897 & 0.2034 & -0.0058 & 0.035 \\ \text { H23 } & 0.2105 & 0.2913 & -0.1106 & 0.041 \\ \text { H24 } & 0.1160 & 0.3840 & -0.1695 & 0.041 \\ \text { H25 } & 0.0026 & 0.3885 & -0.1229 & 0.036 \\ \text { H33 } & -0.2410 & 0.3696 & -0.1795 & 0.043 \\ \text { H34 } & -0.2523 & 0.5123 & -0.1296 & 0.047 \\ \text { H35 } & -0.1718 & 0.5605 & -0.0249 & 0.049 \\ \text { H36 } & -0.0792 & 0.4648 & 0.0300 & 0.042 \\ \text { H37A } & -0.1576 & 0.1932 & -0.1000 & 0.055 \\ \text { H37B } & -0.0953 & 0.2294 & -0.1514 & 0.055 \\ \text { H37C } & -0.1805 & 0.2318 & -0.1855 & 0.055 \\ \text { H43 } & 0.2306 & -0.0461 & 0.0952 & 0.046 \\ \text { H44 } & 0.2950 & 0.0146 & 0.2046 & 0.050 \\ \text { H45 } & 0.2558 & 0.1489 & 0.2561 & 0.049 \\ \text { H46 } & 0.1537 & 0.2230 & 0.1940 & 0.041 \\ \text { H47A } & 0.1316 & 0.0337 & -0.0409 & 0.061 \\ \text { H47B } & 0.0594 & 0.0232 & 0.0044 & 0.061 \\ \text { H47C } & 0.1195 & -0.0554 & 0.0077 & 0.061 \\ \text { H1S } & 0.5180 & 0.1882 & 0.2847 & 0.046\end{array}$

